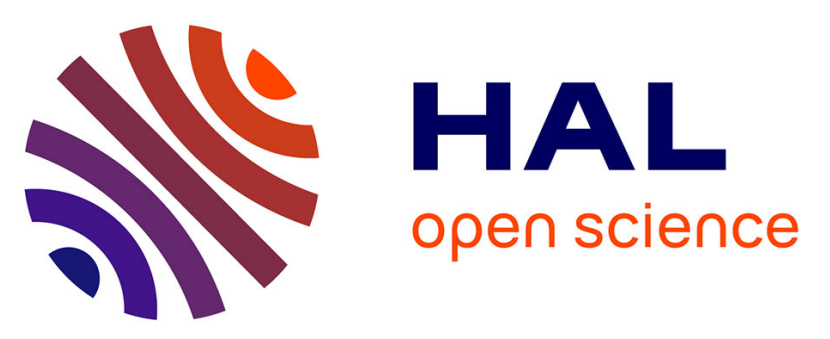

\title{
Bicomponent Supramolecular Architectures at the Vacuum-Solid Interface
}

Xavier Bouju, Cristina Mattioli, Grégory Franc, Adeline Pujol, André Gourdon

\section{- To cite this version:}

Xavier Bouju, Cristina Mattioli, Grégory Franc, Adeline Pujol, André Gourdon. Bicomponent Supramolecular Architectures at the Vacuum-Solid Interface. Chemical Reviews, 2017, 117 (3), pp.1407-1444. 10.1021/acs.chemrev.6b00389 . hal-01737665

\section{HAL Id: hal-01737665 \\ https://hal.science/hal-01737665}

Submitted on 19 Mar 2018

HAL is a multi-disciplinary open access archive for the deposit and dissemination of scientific research documents, whether they are published or not. The documents may come from teaching and research institutions in France or abroad, or from public or private research centers.
L'archive ouverte pluridisciplinaire HAL, est destinée au dépôt et à la diffusion de documents scientifiques de niveau recherche, publiés ou non, émanant des établissements d'enseignement et de recherche français ou étrangers, des laboratoires publics ou privés. 


\section{Bicomponent supramolecular architectures at the}

\section{vacuum-solid interface}

Xavier Boujuł, Cristina Mattioli†, Grégory Franct, Adeline Pujolł, André Gourdon†**

†CEMES-CNRS, 29 Rue J. Marvig, 31055, Toulouse, France

$\$$ †niversité de Toulouse, UPS, CNRS, CEMES, 118 route de Narbonne, 31062 Toulouse, France

KEYWORDS: Bicomponent self-assembly, STM, vacuum-solid, surface, UHV

CONTENTS

1. Introduction

1.1. Bicomponent self-assembly on surfaces

1.2. Vacuum-solid versus liquid-solid

2. Bonds and interactions

2.1. Inter-molecular interactions

2.1.1. van der Waals interactions

2.1.2. Hydrogen bonding

2.1.3. Halogen bonding

2.1.4. Intermolecular $\pi-\pi$

2.1.5. Electrostatic interactions

2.2. Role of the surface 
2.2.1. Surface molecule van der Waals interactions

2.2.2. Charge transfer

2.2.3. Electrostatics

2.2.4. $\pi-\pi$ interaction on $\mathrm{HOPG}$

2.2.5. Substrate-mediated interactions and other related effects

2.2.6. Metal organic

2.2.7. Image charge

2.2.8. Crystalline faces

2.2.9. Comparison between adsorption on noble $\mathrm{Cu}, \mathrm{Ag}$, and $\mathrm{Au}$ surfaces

2.2.10. Diffusion barriers

2.2.11. Molecule-induced surface reconstruction

2.2.12 Résumé of the various interactions responsible for self-assembly

3. Self-assembly by directional interactions: complementary H-bonding moieties

3.1. Melamine-based structures

3.1.1. Melamine and cyanuric acid

3.1.2. Melamine and dicarboxylic imides

3.1.3. Melamine and PTCDA

3.2. Other dicarboxylic imide/anhydride/acid-based self-assembled systems

3.2.1. PTCDI or NTCDI and guanamine derivatives

3.2.2. PTCDA and amines

3.2.3. Carboxylic acids

3.3. Biology-related compounds

4. Metal-ligand coordination 
5. Assemblies stabilized by non-directional interactions

\subsection{Porphyrins}

5.2. Phthalocyanine: penta-tert-butyl-corannulene

5.3. Phthalocyanine: DIP/pentacene

5.4. Sub-phthalocyanine

\subsection{TTF-TCNQ}

5.7. $\mathrm{C}_{60}$ and pentacene

\section{Outlook}

6.1 Towards predicting bicomponent self-assembly: simulations

6.2 Towards higher complexity: multicomponent systems

6.3 Non-metallic surfaces

6.4 Nanopores

6.5 Organic thin films

\section{Conclusion}

Author Information

Corresponding Author

Biographies

Acknowledgments

Glossary

References 


\section{INTRODUCTION}

"I do not believe in things, but in the relations in-between things" ("Je ne crois pas aux choses, mais aux relations entre les choses"). ${ }^{1}$ These were the words of the painter Georges Braque in a definition of imagination. This sentence may be taken as the basis for self-assembly. Consider a set of basic elements and a library of interaction forces between them with both attractive and repulsive components; the overall system will then generally evolve to form more or less complex structures. In nature, many examples involving various kinds of interaction forces can be encountered at various length scales. Complex self-assembled patterns generated by collective motions, such as bird flocks or fish schools ${ }^{2}$ or colonies of ants, ${ }^{3}$ are more or less spontaneously formed. Such adaptive behavior was recently simulated by a swarm of cooperative and programmed robots in a two-dimensional (2D) space to achieve shape formation. ${ }^{4}$ At a smaller scale, the dynamics of self-assembled patterns with cell and bacterial colonies is governed by physical and biophysical interactions between units. ${ }^{5}$ In particular, self-assembled Escherichia coli on a $2 \mathrm{D}$ support responds to biomechanical forces. ${ }^{6}$

At the molecular level, cooperativity is generally expressed with forces that originate from electromagnetism. A wide and active field of research focuses on the self-assembly of large molecular systems, such as viruses ${ }^{7}$ and peptides. For example, bio-inspired nanomaterials, such as silica nanotubes, ${ }^{8}$ peptide nanotubes, ${ }^{9,10}$ and DNA origami, ${ }^{11}$ have been obtained via selfassembly. One of the most active themes in this field has been the exploration of self-assembly at surfaces involving smaller molecules, ${ }^{12,13,14,15,16,17,18,19}$ and this subject will constitute the main topic of the present review, with the focus on self-assembly on surfaces in high vacuum involving two different molecular species. It is expected that this chemical bottom-up strategy 
will allow the engineering of functional materials with sub-nanometer precision via selforganized growth in a fast and parallel fashion to yield relatively robust materials with selfhealing properties. ${ }^{20}$ This objective is driven by a variety of potential applications in growing fields such as molecular scale electronics, sensing, catalysis, organic photovoltaic (OPV) cells, organic spin valves, and optoelectronics.

\subsection{Bicomponent self-assembly on surfaces}

Although the presence of a surface limits the potential to form a wide variety of self-assembled three-dimensional (3D) structures, it provides a spatial constraint that opens routes for other selfassembly patterns in 2D. Binary compounds are drawing increasing attention because they offer more flexibility to trigger and control assembly. Structural growth is governed by both the type of interaction and the respective amounts of the two species. Experimental and theoretical studies of monocomponent self-assembled systems on surfaces have already yielded substantial information on intermolecular and molecule-substrate interactions; using two molecular components potentially offers more flexibility for the design and synthesis of complementary complex supramolecular systems that can self-organize on surfaces in a controlled way. Multicomponent self-assembly with three or more species would also be of great interest; however, as described below in section 6.2 and considering the restrictions defined at the end of this introduction, the number of such systems is still limited.

\subsection{Vacuum-solid versus liquid-solid interfaces}

Apart from the pioneering work of Ohtani et al. in $1988^{21}$ and certain recent studies, the vast majority of bicomponent molecular systems at surfaces have been studied at the liquid-solid 
interface, mainly for the following reason: as scanning tunneling microscopy (STM) is currently the tool of choice for characterizing supramolecular architectures, it is much cheaper, much faster and more convenient to carry out experiments in liquids on easily prepared surfaces such as highly oriented pyrolytic graphite (HOPG), $\mathrm{MoS}_{2}$ or gold. ${ }^{22,23,24,25,26,27,28,29}$ The transfer of molecules to the surfaces only requires sufficient solubility in a solvent such as phenyloctane, octanoic acid, an alkane, 1,2,4-trichlorobenzene, or a dilute acid, among others. Additionally, molecule dosing is also conveniently performed by controlling the solute concentration, and all experiments can be carried out in air at approximately room temperature (RT) using relatively inexpensive tools. A second appealing aspect is the possibility of operating under equilibrium conditions close to those observed in solution and to mimic phenomena observed in Nature. The substrate is in general not highly involved in intermolecular interactions, so many concepts of supramolecular chemistry in solutions can be applied as a first approximation.

In the past ten years, the interest in supramolecular assembly at vacuum-solid interfaces has increased considerably for the following reasons:

a) Whereas at liquid-solid interfaces the number of suitable surfaces is essentially limited to graphene, $\mathrm{HOPG}, \mathrm{MoS}_{2}, h$-BN, mica and gold, nearly any crystalline conducting or semiconducting substrate can be used in ultra-high vacuum (UHV), including reactive and/or structured surfaces, thin films on metal, etc. The development of UHV non-contact atomic force microscopy (NC-AFM) is expected to extend this list of accessible surfaces to insulating substrates and large band-gap semiconductor surfaces.

b) At RT or below, the desorption of molecules generally occurs at a low rate, with higher adsorption energy and with limited ${ }^{30}$ desorption/re-adsorption processes as observed in solution 
by exchange with the solvent. Furthermore, at high coverage, the mass transport is not high, so that in some cases non-equilibrium assemblies can be stable.

c) Although the dosing of molecules is more complex than in solution, it is nevertheless more controllable: the quantity of deposited material can be measured by quartz-microbalance, and the two types of molecules can be evaporated successively or by simultaneous co-evaporation using crossed beams. The difference in adsorption energies is therefore a less important parameter than at the liquid-solid interface, where the weakly adsorbed component can be substituted by a strongly adsorbed component, with a direct impact on the relative ratio of the two species on the surface. Furthermore, in concentrated solutions, molecules can stack as multilayers. For instance, it has been shown using atomic force microscopy (AFM) that trimesic acid (TMA) films at the $\mathrm{H}_{2} \mathrm{O} / \mathrm{HOPG}$ liquid-solid interface can form 3-5 multilayers ${ }^{31}$ that cannot be detected by STM, which can only image the first monolayer in contact with the substrate. This phenomenon is likely to be a common one, but so far, it has not been fully studied. ${ }^{32,33,34}$

Current developments of adapted electrospray and soft-landing techniques now permit the transfer of essentially any molecule or salt from solution to $\mathrm{UHV}^{35,36,37,38,39}$

d) The spatial resolution of UHV-STM (and now NC-AFM) is much higher than in solution, allowing precise characterization of sub-atomic details and conformations and precise measurements of interatomic distances. STM manipulation of single atoms and molecules is now a routine and precise technique that allows perturbation of the observed system to evaluate its stability and to displace molecules.

e) Many UHV complementary surface spectroscopy techniques are available: scanning tunneling spectroscopy (STS), X-ray photoelectron spectroscopy (XPS), photoelectron spectroscopy 
(PES), angle-resolved photoelectron spectroscopy (ARPES), near-edge X-ray-absorption fine structure (NEXAFS), helium scattering, and Kelvin probes, among others.

f) In UHV, the absence of a solvent (and therefore of solvent-substrate and solvent-molecule interactions involving adsorbed gases) permits cleaner and simpler systems for which better numerical simulations are possible that take into account only the substrate and the molecules.

g) In the absence of a solvent, the range of temperature that can be used for deposition, annealing and imaging steps is much wider, ranging from $4 \mathrm{~K}$ to $500 \mathrm{~K}$.

h) The resulting self-assembly can be significantly different at liquid-solid and vacuum-solid interfaces. For instance, when tetrathiafulvalene (TTF) derivatives functionalized with lateral dodecylthio chains are sublimated onto graphene/SiC(0001) in $\mathrm{UHV},{ }^{40}$ the "fastener effect" induced by van der Waals (vdW) interactions between the alkylthio chains forces the TTF to self-organize in a $\pi-\pi$ stacked edge-on conformation similar to what is observed in the molecular crystal. In contrast, at the liquid/solid interface, the TTF lies flat on the surface (face-on conformation) as the solvent is involved in the stabilization of the molecular layer.

i) Finally, perhaps surprisingly, the exploration of molecular self-assembly on surfaces in UHV can be considered more suitable for and closer to future technologies. Important recent progress has been achieved for organic light-emitting diodes (OLEDs), organic field-effect transistors (OFETs), OPV cells, and organic spintronics, ${ }^{42}$ among other devices. The development of such devices requires precise control of the electronic properties of the substrate. On-surface selfassembly allows merging the high carrier mobility of the surface with the advantages of organic films in terms of cost, processing and tunability. ${ }^{43}$ Bottom-up self-assembly on ultra-clean surfaces is the method of choice to scatter and confine surface states, mix with them, and modify the energy level alignment at the interface and the charge carrier injection in the heterojunction. 
In these systems, bicomponent self-assembly is one of the crucial ingredients because it allows the preparation of complex structures that would be exceedingly difficult to synthesize in solution and then transfer in ultra-clean conditions to a substrate. For instance, it was reported that a photovoltaic device consisting of a heterojunction based on $\mathrm{C}_{60}$ as the acceptor and copper phthalocyanine as the donor can reach efficiencies of approximately $5.0 \%{ }^{44}$

This review is limited to systems for which both components play a key role in the formation of 2D supramolecular architectures. Therefore, we will not discuss in detail the entrapment of guests in preformed stable supramolecular voids or nanoporous networks ${ }^{45,46,47,48,49}$ or systems with admolecules at the top of a supramolecular layer. ${ }^{50}$ Although they could be considered bi- or multicomponent supramolecular systems, these types of networks will be briefly mentioned only in the context of perspectives.

In the first part, we introduce the various interactions present in the structures of heteromolecular monolayers that are discussed in this article. The review will then survey the different types of bicomponent systems based on their intermolecular interaction energies, i.e., starting from strong directional multiple H-bonding to vdW physisorption.

\section{BONDS AND INTERACTIONS}

The structure of a molecular overlayer on a substrate is the result of a balance between molecule/molecule and molecule/surface interactions. ${ }^{51,52,53,54,22,55,56,45,57,58,59,60,61,62,63}$ According to the relative strengths of these interactions and the presence of a solvent, ${ }^{64}$ various geometries can be observed. To a first approximation, two different cases are observed: when the molecular surface interaction is strong, the symmetry of the substrate dictates the molecular layer structure; 
alternatively, in the case of strong directional intermolecular bonds, the molecular layer and the surface are independent from each other with their own organizations. However, as we will discuss, this picture is only an approximation; many other interactions come into play.

\subsection{Intermolecular interactions}

Before describing molecule/molecule and molecule/surface interactions, we will summarize the universal form of atom/atom interaction, which is the summation of a repulsion and an attraction expressed as $V(R)=V_{\text {rep }}(R)+V_{\text {att }}(R)$, where $R$ is the distance between atom $A$ and atom $B$. Here, $V_{\text {rep }}$ is a repulsive potential that can be expressed with a generic form such as a BornMayer form $a \exp (-b R)$ with $a$ and $b$ as two adjustable parameters, or with a $c_{n} / R^{n}$ term with $9 \leq n \leq 12$. This contribution originates from the first-order Coulomb and exchange energies between electrons for covalent bonding with an opposite sign and dominates the potential at small distances. In the context of the present review, we will not proceed beyond this contribution. ${ }^{65}$ The $V_{a t t}(R)$ term accounts for the attractive interactions ranging from covalent attraction to dispersion interaction. ${ }^{66}$ For example, one of the general forms of this later longrange dispersion energy can be written as the series $V_{a t t}(R)=-\sum_{n \geq 3} \frac{C_{2 n}}{R^{2 n}}$ with $C_{6}=C(1,1)$, $C_{8}=C(1,2)+C(2,1), \quad$ and $\quad C_{10}=C(1,3)+C(2,2)+C(3,1), \quad$ where $C\left(l_{1}, l_{2}\right)=\frac{\left(2 l_{1}+2 l_{2}\right) !}{4\left(2 l_{1}\right) !\left(2 l_{2}\right) !}\left(\frac{2}{\pi}\right) \int_{0}^{\infty} \alpha_{l_{1}}^{A}(i \omega) \alpha_{l_{2}}^{B}(i \omega) d \omega .{ }^{65}$ The key quantity is $\alpha_{l}^{A / B}$, which is the $2^{l}-$ pole dynamic polarizability of atom $A / B$ ( $l=1$ represents a dipole, $l=2$ a quadrupole, $l=3$ an octopole, $l=4$ a hexadecapole...). In the case of three or more atoms, there exists a nonadditivity characteristic arising for the $N$-body interactions. ${ }^{67,68}$ Such contributions are generally weak, except for specific nanostructured systems, and the total vdW interaction between two 
molecules is calculated in a first approximation by summing all possible pairs of atoms and excluding the interactions inside each molecule.

Two-dimensional self-assembly is thus governed by the strength of the molecule/molecule interactions, which provides a hierarchy of structures stabilized by an attraction/repulsion balance at equilibrium. Generally, weak intermolecular interactions generate close-packed molecular layers, whereas directional self-assembly is mainly produced by strong intermolecular interactions. ${ }^{69}$ Without being exhaustive, we now describe briefly some of these molecule/molecule forces from weakest to strongest.

\subsection{1 van der Waals interaction}

The vdW interaction term is a generic appellation gathering various kinds of contributions: ${ }^{70}$ the Keesom contribution (between two molecules with fixed dipoles), the Debye term (between a rotating dipole and a non-polar molecule), and the London energy or the dispersion energy (between two instantaneous dipoles). These three terms have a $1 / R^{6}$ dependency according to the distance. Among these terms, only the Keesom contribution describes a directional interaction.

For large molecules, there are numerous systems in which the long-range vdW interactions are responsible for self-organization on a surface, as for instance, in the case of the

self-assembly of jointed molecules consisting of Lander molecules ${ }^{71}$ on a metallic substrate ${ }^{72,73}$ or hydrocarbonated or acidic molecules on graphite. ${ }^{74,75,76,77}$

\subsubsection{Hydrogen bonding}

Among the various interactions between molecules, hydrogen bonding (H-bonding) is probably the most difficult interaction to define and characterize. It is not the purpose of this review to 
enter the debate on the origin of H-bonding. For an official definition, refer to the IUPAC international committee texts. ${ }^{78,79}$ Briefly, the most familiar picture of the H-bond is X-H $\Lambda \mathrm{Y}$, where a hydrogen atom is situated between two atoms $\mathrm{X}$ and $\mathrm{Y}$. These two atoms are electronegative with negatively charged character. According to the nature of these two atoms, i.e., their relative electronegativities, many hydrogen-bond types can be observed, ranging from strong to weak H-bonding. ${ }^{80,81,82,83,84,85}$ With an energy of $11.5-23 \mathrm{kcal} / \mathrm{mol}(0.5-1 \mathrm{eV})$, as observed for the conventional $\mathrm{N}-\mathrm{H} \Lambda \mathrm{O}$ bond, for instance, the $\mathrm{H}$-bonds are considered strong; they are moderate for energies in the range $0.5-11.5 \mathrm{kcal} / \mathrm{mol}(0.2-0.5 \mathrm{eV})$ and weak below 0.5 $\mathrm{kcal} / \mathrm{mol}(0.2 \mathrm{eV})$.

Generally, the H-bond is considered a directional interaction (in contrast to the vdW interaction). Due to its properties and in the case of multiple H-bonding, the concepts of attachment points and molecular recognition can explain assembly behavior.

Moreover, relatively exotic or unusual H-bonds can be involved in self-assembled motifs: using the interaction between $\mathrm{H}$ atoms in cyano groups and benzene rings, Yokoyama et al. demonstrated the formation of molecular chains and supramolecular aggregates with controlled shape and size. ${ }^{86}$ Directional self-assembly was also described in the case of anthraquinone molecules on the $\mathrm{Cu}(111)$ surface with a H-bond between a carbonyl oxygen and an aromatic ring hydrogen atom. ${ }^{87}$

Additionally, a particular class of interaction, usually classified as a weak H-bond and a type of $\pi$ interaction, is the $\mathrm{CH}-\pi$ interaction. ${ }^{88,89,90}$ It consists of the interaction between a $\mathrm{CH}$ bond and a $p_{z}$ orbital belonging to an aromatic molecule. This interaction exhibits some directional character, which favors linear self-assembled structures. The strength of this 
interaction is on the order of a few $\mathrm{kcal} / \mathrm{mol}$, so that the stability of one-dimensional (1D) patterns on a surface is relatively small except when multiple $\mathrm{CH}-\pi$ bonds are engaged.

In molecular modelling calculations, H-bonds can be implemented in different ways. There are various molecular mechanics packages with specific force fields, such as the MM3MM4 force field, ${ }^{91,92}$ Dreiding, ${ }^{93}$ PCFF, ${ }^{94,95}$ Amber, ${ }^{96,97}$ and CHARMM. ${ }^{98}$ In the MM3-MM4 code, for instance, the H-bond is described by a set of parameters and a specific formulation that takes into account the H-bond angularity and the lone-pair directionality. On the other hand, density functional theory (DFT) methods are adapted to address H-bonding. This latter technique allows precise self-consistent calculations of the electronic density around the atomic centers responsible for the H-bonding and has been applied and checked for small hydrogen-bonded complexes. $^{99,100,101}$ Some force fields underestimate the H-bond interaction compared to DFT results and have to be reparametrized. ${ }^{102}$ DFT self-assembly studies are limited to relatively small systems supported by a surface, such as adenine pairs on $\mathrm{Au}(111) .^{103}$

\subsubsection{Halogen bonding}

The halogen bond (X-bond) originates from the attractive interaction between "an electrophilic region associated with a halogen atom in a molecular entity and a nucleophilic region in another, or the same, molecular entity", as defined by the IUPAC. ${ }^{78}$ More precisely, this interaction appears between a halogen atom, which is covalently connected to a group and has an electron-poor region (donor) and an acceptor molecular group with an electron-rich region. Similar to H-bonding, X-bonding can also direct assembly efficiently. ${ }^{104,105,106}$ For a general review on X-bonding in supramolecular chemistry, see Beer et al. ${ }^{107}$ and Cavallo et al. ${ }^{108,109}$ The $\mathrm{X}$-bond is an alternative to the H-bond and presents similar properties, especially in terms of directional bonding. It has already been exploited to form porous networks on 
$\operatorname{Ag}(111)^{110}$ and well-ordered supramolecular networks on oxidized $\mathrm{Cu}(110)$ surfaces $^{111}$ as well as $\mathrm{Cu}(111),{ }^{112} \mathrm{Au}(111),{ }^{113} \mathrm{Ag}(111),{ }^{114} \mathrm{HOPG},{ }^{115,116,117}$ and $\mathrm{SiB}(111)$ surfaces. ${ }^{118}$

\subsubsection{Intermolecular $\pi-\pi$}

The $\pi-\pi$ interaction is a general term for the interaction between two aromatic molecules, that is, two $\pi$-electron-rich molecules. This type of interaction is particularly important in many areas in biology and chemistry. ${ }^{119,120}$ The origin and strength of the $\pi$ interaction have generated lively discussions in the scientific community (and continue to feed a fertile debate). On the basis of an electrostatic model, Hunter and Sanders have described interacting molecules as a set of point charges supported by atomic sites. ${ }^{121}$ Typically, each atom of the $\sigma$-skeleton has a charge of +1 and two charges of -0.5 separated by a particular distance ( $0.47 \AA$ for carbon). This model provides reasonable information and qualitative rules for different geometries in regards of the orientation of the two molecules and can be applied to proteins. ${ }^{122}$ The simplest example to illustrate such an interaction between arene rings is the benzene-benzene case. In particular, the sandwich (face-to-face), T-shaped or parallel displaced (face-to-face displaced) configurations have been theoretically studied. The empirical Hunter and Sanders rules specify that $\pi-\sigma$ attraction is responsible for the T-shaped and parallel displaced geometries, whereas $\pi-\pi$ repulsion disfavors the sandwich configuration. Moreover, this prototype example allows the use of sophisticated methods, such as high-level $a b$ initio quantum mechanical computations, ${ }^{123,124,125,126,127}$ to study the $\pi$ interaction more qualitatively (the binding energy ranges between $2.8 \mathrm{kcal} / \mathrm{mol}$ for the parallel-displaced configuration and $1.8 \mathrm{kcal} / \mathrm{mol}$ for the sandwich configuration). Nevertheless, according to the degree of applied corrections in these 
methods, the results show large amplitudes of variations, and the balance between the dispersion contribution and the $\pi$ interaction is not definitively established. ${ }^{128}$

In terms of self-assembly, the directionality of the $\pi-\pi$ interaction favors the growth of stacked linear structures. This finding was observed for different molecules, which are stacked in one direction on the surface. According to the interplay with the surface interaction, aromatic molecules are thus able to self-assemble parallel to the surface, ${ }^{73,129}$ forming molecular columns, or perpendicularly by $\pi$ stacking, ${ }^{64,130}$ generating molecular filaments on the surface.

\subsubsection{Electrostatic interactions}

The presence of permanent charges or permanent dipoles/quadrupoles on a molecule generates an electrostatic field that influences the generation of $2 \mathrm{D}$ films. Let us distinguish the selfassembly of ions, zwitterions, and donor-acceptor compounds.

In the case of molecules with ionic character, a construction with alternate anions and cations is often found. For instance, co-adsorption of 7,7,8,8-tetracyanoquinodimethane (TCNQ) and Mn atoms on $\mathrm{Cu}(100)$ leads to dianions of $\mathrm{TCNQ}^{2-}$ interacting with $\mathrm{Mn}^{4+}$ cations or $\mathrm{TCNQ}^{1-}$ with $\mathrm{Mn}^{2+}$ cations. $^{131}$

Zwitterions constitute a class of neutral molecules exhibiting charged centers, which are usually designed as acceptor (electron-withdrawing group) and donor (electron-rich group) entities, such as "push-pull" molecules or $\pi$-conjugated zwitterions. ${ }^{132}$ Such molecules strongly adsorb on ionic crystal surfaces by electrostatic interactions; for instance, the 4-methoxy-4'-(3sulfoatopropyl)stilbazolium molecule terminated by a negatively charged sulfonato group linked to a pyridinium positive ring adsorbs strongly on the negatively charged anion of ionic surfaces, such as $\mathrm{KCl}, \mathrm{RbCl}$, and $\mathrm{KBr}$ substrates, with its electric dipole perpendicular to the substrate 
surface. ${ }^{133}$ Similar behavior is also observed on metallic surfaces ${ }^{134,135}$ and semiconducting substrates. $^{136,137,138,139}$ However, the complexity of managing the driving forces to generate bicomponent complexes with two zwitterions is substantial; to our knowledge, there is currently no example of bicomponent systems comprising two zwitterions.

Intermolecular contributions arising from electron donor-acceptor dyads should also be considered in the present discussion. Briefly, charge transfer in a binary donor-acceptor complex can occur that modifies the intermolecular interaction. ${ }^{140,141}$ For instance, it was recently proposed that such a phenomenon generates a change in the H-bonding between $\mathrm{Cu}$ phthalocyanine ( $\mathrm{CuPc}$, donor) and $\mathrm{F}_{16} \mathrm{CuPc}$ (acceptor) via a gap-mediated interfacial charge transfer on $\mathrm{HOPG}^{142}$ or a direct charge transfer inside the sexithiophene (donor) — tetrafluorotetracyano-quinodimethane (acceptor) structure on $\mathrm{Au}(111) .{ }^{143,144}$ An elegant example is the mixing of $\mathrm{C}_{60}$ (acceptor) and porphyrins (donor) on $\mathrm{Ag}(110)$ reported by Sedona et al. ${ }^{145}$ Depending on the tetraphenylporphyrin functionalization and the stoichiometry, the selfassembled monolayer structure varies, giving rise to phases of stripes or pores.

Dipolar interactions arise when two molecules exhibit a permanent dipole. For instance, for two dipoles of $1 \mathrm{D}$ (recall that a water molecule has a permanent dipole of $1.85 \mathrm{D}$ ) separated by $0.4 \mathrm{~nm}$, the dipole-dipole interaction is approximately $20 \mathrm{meV}(0.46 \mathrm{kcal} / \mathrm{mol})$ when the two dipoles are aligned. Such an interaction is thus generally weak and varies as $1 / r^{3}$. Interestingly, this interaction was implicated as the driving force in the formation of the $2 \mathrm{D}$ porous honeycomb structure of hexa-peri-hexabenzocoronene ( $\mathrm{HBC}$ ) functionalized by $\mathrm{CF}_{3}, \mathrm{CN}$, or $\mathrm{NO}_{2}$ groups on HOPG. ${ }^{146}$ The anti-parallel dipole-dipole interaction between $\mathrm{HBC}-\mathrm{CF}_{3}$ is sufficiently high with respect to the vdW interactions alone to form such 2D structures. Additionally, extra H-bonding appears to be necessary to form nanoporous structures with $-\mathrm{CN}$ and $-\mathrm{NO}_{2}$ moieties. Similarly, 
but on a metallic surface, anti-parallel $[\mathrm{CN} \Lambda \mathrm{NC}]$ dipolar interactions and weak $\mathrm{CN} \cdots \mathrm{HC}_{\mathrm{sp} 2} \mathrm{H}-$ bonding favor the formation of chains from the adsorption of trans-bis(4'cyanobiphenyl)porphyrins on a $\mathrm{Cu}(111)$ surface. ${ }^{147}$ Aesthetically appealing figures are formed with three to six molecules, as are long linear branched chains.

A hexagonal porous network was observed recently in a study of hexaaza-triphenylenehexacarbonitrile molecules on $\mathrm{Au}(111) .{ }^{148}$ Here, dipolar interactions involving two $\mathrm{CN}$ groups are responsible for linear structures along the step-edge and a 2D nanostructure on terraces. Three CN $\Lambda \mathrm{CN}$ dipolar interactions are involved, and DFT calculations indicate that an energy of $70 \mathrm{meV}(1.6 \mathrm{kcal} / \mathrm{mol})$ is found per bond.

Additionally, a quadrupolar interaction, even though it is weaker than the dipolar one, explains the formation of a self-assembled film on a gold surface with functionalized arenethiols. Bartels et al. showed how thiophenol molecules and certain specifically substituted derivatives (arenethiols with p-bromo-, p-chloro-, p-fluoro-, and pentafluoro-substituted analogues) can tune the film properties on $\mathrm{Cu}(111) .{ }^{149}$ The change of substituent modifies the electronegativity of the ending group, which is found to accommodate the intermolecular interaction via the molecular quadrupole moment.

\subsection{Role of the surface}

As mentioned previously, the other component directing self-assembly on a surface is clearly the molecule/surface interactions. The adsorption is generally classified between chemisorption and physisorption, dealing with strong to weak interactions. However, the frontier between the two is fuzzy, and there is no clear criterion to differentiate the strength of the interactions, although the word chemisorption is always used when chemical bonds are created upon adsorption. In any 
case, when a molecule approaches a metallic surface, various phenomena occur according to the nature of the surface. At a certain distance, the adsorbate orbitals can be hybridized with the surface states, which generates either a positive or a negative coupling. This effect is mediated by the pillow effect, energy level alignment, induced density of interface states, charge transfer, and generation of separate bonding and antibonding orbitals at the molecular level. ${ }^{150,151,152,153,154}$ As clearly explained by Goiri et al. ${ }^{63}$ and by Kolasinski, ${ }^{155}$ the occupancy of the bonding and the anti-bonding states governs the nature of the bond; if the bonding states are filled, the bond strength is strong (i.e., the interaction is more attractive) and if anti-bonding is partially or fully filled, the bond is weaker (i.e., the interaction is less attractive). This state filling is related to the d-band position of the metal with respect to the Fermi energy: for noble metals, the anti-bonding state is fully filled, so that chemisorption is not favored. Following Hammer and Nørskov, ${ }^{156,157}$ it is then possible to explain why early transition metals react more strongly than transition metals at the right end of a row in the classification: as the d-band size increases for $\mathrm{Cu}(3 \mathrm{~d}), \mathrm{Ag}$ (4d), and $\mathrm{Au}(5 \mathrm{~d})$, the interaction decreases, and the least reactive metal (or the noblest one) is thus gold. In the following, we will focus on non-chemisorbed interactions between molecule and surface that are more favorable for self-assembly.

\subsubsection{Surface molecule van der Waals interactions}

To calculate the vdW contribution between a molecule and a surface or between a molecule and its neighboring molecules, it is necessary to address the many-body character of this energy. Recently, several DFT-based methods to treat vdW contributions have been developed, ${ }^{158,159,160,161}$ including many-body contributions. ${ }^{162,163}$ 
Among the various existing theories, the coupled-modes method ${ }^{164}$ provides a convenient framework for describing the dispersion energy between a molecule and a surface. In short, the coupled modes between the systems in interaction are the solution of a so-called dispersion relation, which includes the dipolar polarizability of each interacting atom (see details in Ref. ${ }^{165}$ and 166 for the description of the iterative scheme for calculating the many-body vdW contribution). This procedure allows the calculation of the dispersion energy between an assembly of physisorbed molecules at the many-body order, not only with polarizable systems but also with a permanent dipole distribution or with higher poles such as quadrupoles by taking into account the presence of the surface atoms. All these techniques are formally able to address arbitrarily shaped surfaces (that is to say, various defects on the surface can be incorporated in the system), and the sole requirement is the computational power available to tackle such calculations.

\subsubsection{Charge transfer}

The charge transfer (CT) between a donor and an acceptor may be modified by the presence of the substrate. When two molecules approach a metallic surface, a broadening of the frontier orbitals and an alignment of the energy levels of the molecules with respect to the surface states generate an induced density of interface states, and a charge transfer between molecules and the

surface is observed. These effects induce a change in the sorption state. ${ }^{167}$ Additionally, such a charge transfer induces a distortion of the underlying reconstructed surface, and a surfacemediated charge transfer is identified as in the case of the mixing of tetrathiatetracene (TTT, donor) and tetracyano naphtho quinodimethane (TNAP, acceptor) on Au(111). ${ }^{168}$ Similarly, Umbach et al. have shown charge transfer in alternating TNAP/TTF monolayer rows; that is, the 
TNAP rows are charged due to the presence of TTF rows mediated by the Au(111) surface. ${ }^{169}$ Fernández-Torrente et al. have reported that a donor molecule (tetramethyltetrathiafulvalene) provides a charge transfer that is sufficiently high to modify the adsorption state of an acceptor molecule (TCNQ) in stoichiometric mixing on $\mathrm{Au}(111)$, resulting in an electronic decoupling of the acceptor from the substrate. ${ }^{170}$

\subsubsection{Electrostatics}

The adsorption of metallic atoms on a metallic surface directs the structure of a self-assembled monolayer which then depends on the charge state of the adatom. If the metal has a natural ability to become an anion, such as alkali metal atoms with low electronegativities and ionic potentials, molecules with an apparent ionic character interact through these linkers. For instance, Stepanow et al. used cesium adatoms with four polyaryl dicarboxylic acids, which produces various chiral arrangements of the network structures. ${ }^{171}$ However, a naive electronegativity argument does not generally apply for all metallic adsorbates because the anisotropy experienced by surface atoms induces a modification in the relative scale of electronegativity factors. ${ }^{172}$

Another case corresponds to the change in the charge state upon adsorption on the metallic surface. As an example, co-deposition of $\mathrm{Ni}$ atoms and perylene tetracarboxylic diimide (PTCDI) molecules produces three distinct types of nanostructures as a function of the $\mathrm{Ni}$ PTCDI coverage. ${ }^{173}$ Here, the Ni adatoms acquire a negative partial charge through interaction with the substrate, and the Ni-PTCDI interaction is entirely electrostatic, which is consistent with the 18-electron rule for transition metal complexes. ${ }^{174}$ A surface coordination network involving negatively charged metal centers was reported ${ }^{175}$ in the case of $\mathrm{Cu} / \mathrm{Cu}(111)$, in which it was 
shown that a $\mathrm{Cu}$ adatom surrounded by three 9,10-anthracenedicarbonitrile molecules exhibits an excess of negative partial charge to remedy electron donation from the substrate to the ligand.

Thus far, these studies have been limited to atom/molecule binary systems, and ternary systems comprising two molecular species and one metallic adatom remain to be explored. Such experiments would shed light on the influence of electrostatic parameters on bicomponent supramolecular self-assembly.

\subsection{4 $\pi-\pi$ interactions on HOPG}

Molecular self-assembly on graphite and now on graphene has attracted considerable attention due to the ease of sample preparation, especially on HOPG, and of STM characterization. ${ }^{176}$ Many studies have focused on the adsorption of aromatic molecules that adsorb in a flat manner and follow the AB stacking of the graphite/graphene substrate. The adsorbed molecules may then act as a local dopant by charge transfer, and/or the $\pi-\pi$ stacking contributes to modify the electronic properties of graphene in a more or less controlled way, ${ }^{177,178}$ as in the case of PTCDI and melamine on epitaxial graphene on $\mathrm{SiC}(0001)$, in which a nanoporous scaffold was generated in UHV. ${ }^{179}$

\subsubsection{Substrate-mediated interaction and other related effects}

The (111) face of noble metal surfaces is the site of phenomena involving free electrons and the formation of a specific surface state called the Shockley surface state. These free surface-state electrons are decoupled from the bulk electrons; they form an electron sea with a free-particlelike dispersion. This phenomenon was observed in real space using STM through surface wave patterns (Friedel oscillations) generated on the surface due to the presence of defects. ${ }^{180,181,182,183}$ The most spectacular example is the formation of electronic standing waves inside a so-called quantum corral. $^{184,185}$ 
Interestingly, the surface electrons can participate in molecule-molecule interactions through so-called substrate-mediated interactions. In fact, a single adsorbate can be assimilated to an electron scatterer to perturb the electron sea underneath by generating an electronic surface oscillation with a period determined by the Fermi wave vector. By approaching a second adsorbate, this latter feels an indirect substrate-mediated interaction due to this oscillatory potential. The interaction range regulates an adsorbate-adsorbate distance typically similar to the period of the surface wave oscillations. This phenomenon was observed for atomic adatoms, such as carbon on $\mathrm{Al}(111),{ }^{186} \mathrm{Co}$ and $\mathrm{Cu}$ on $\mathrm{Al}(111),{ }^{187} \mathrm{Cs}$ superlattices on $\mathrm{Ag}(111),{ }^{188}$ and recently, Gd on $\operatorname{Ag}(111) .{ }^{189}$ Between two adsorbates separated by $R$, this substrate-mediated interaction can be expressed as $V_{\text {sub-med }}(R) \propto \frac{-\sin \left(2 q_{F} R+2 \delta\right)}{\left(q_{F} R\right)^{2}}$, where $q_{F}$ and $\delta$ are two associated parameters. $^{180}$

Observations of molecules are less abundant. ${ }^{190,191,192}$ The strength of adatom-adatom interaction is approximately several $\mathrm{meV}$ and is $10-100 \mathrm{meV}(0.2-2.5 \mathrm{kcal} / \mathrm{mol})$ for molecules, which is generally negligible compared to other interactions such as dispersion. Examples include benzene on $\mathrm{Cu}\{111\}^{193,194}$ and on $\mathrm{Au}\{111\},^{195} 5,10,15,20$-tetraphenyl porphyrins $\left(\mathrm{TPPH}_{2}\right)$ on $\mathrm{Cu}(111)$ and $\mathrm{Ag}(111),{ }^{196}$ acetylene on $\mathrm{Cu}(111),{ }^{197}$ a dipolar iridium complex [tris(2phenylpyridine)iridium(III)] on $\mathrm{Cu}(111),{ }^{198}$ pentafluorinated pentacene on $\mathrm{Ag}(111),{ }^{199}$ and Cophthalocyanine $(\mathrm{CoPc})$ on $\mathrm{Cu}(111)$ and $\mathrm{Ag}(111) .{ }^{200}$ Long-range substrate-mediated repulsion has been observed with anthraquinone molecules on $\mathrm{Cu}(111)$, with local H-bonding occurring between neighboring molecules. $^{201}$

Another contribution must sometimes be considered when calculating the adsorption of a molecule $a$. When this molecule is surrounded by other molecules $b$, these molecules can specifically modify the interaction of molecule $a$ with the surface. In other words, the local 
molecular environment is able to reduce the molecule/surface energy. By considering coadsorption of $\mathrm{C}_{60}$ and 1,3,5,7-tetraphenyladamantane (TPAD) on $\mathrm{Au}(111)$, Franke et al. showed that the electronic coupling of $\mathrm{C}_{60}$ with the surface is significantly reduced due to the TPAD network, ${ }^{202}$ which provides electrostatic interactions that lift the fullerene, reducing its interaction with the surface. Similarly, codeposition of diindenoperylene (DIP) and fluorinated copper-phthalocyanines $\left(\mathrm{F}_{16} \mathrm{CuPc}\right)$ molecules on $\mathrm{Au}(111)$ represents an interesting system combining donor and acceptor character, which decreases the molecule/surface interaction compared to separated deposition. ${ }^{203}$ Such an effect has also been observed with copperphthalocyanine (donor) and perfluoropentacene (acceptor) codeposition on noble metal $\operatorname{Ag}(111)$ and $\mathrm{Cu}(111)$ surfaces. ${ }^{204}$

\subsubsection{Metal-organic}

The codeposition of organic molecules and metal atoms has been widely explored over the past decade. Coordination bonding provides the driving force to build highly ordered networks on metallic surfaces from metal adatoms and organic ligands. The metal-organic interaction is weaker than covalent bonding but is sufficiently strong to create extended scaffolds on the surface with corresponding geometry depending on the metal (generally transition metals such as $\mathrm{Cr}, \mathrm{Fe}, \mathrm{Co}, \mathrm{Au}, \mathrm{Cu}, \mathrm{Mn}$, or $\mathrm{Ni}^{59}$ ) and the coordination geometry. A change of the electronic structure of the metallic adatom and the ligand, due to their mutual interaction, can result in a modification of the surface-ligand interaction. This subtle interplay and, of course, the coordination properties (number and angle) of the metal adatom and the deposition ratio can generate a large family of molecular networks on a surface.

Examples include, among many supramolecular surface nanostructures involving coordination bonds, $\mathrm{Cr}$-p-terphenyldinitrile on $\mathrm{Au}(111),{ }^{205} \mathrm{Fe}$-terephthalic acid on $\mathrm{Cu}(100),{ }^{206}, 207$ 
Fe-biphenolate and Co-dicarbonitrile on $\mathrm{Ag}(111),{ }^{208} \mathrm{Fe}$ - and $\mathrm{Cu}$-trimesic acid on $\mathrm{Cu}(110),{ }^{209}$ diiron-diterephthalate on $\mathrm{Cu}(100),{ }^{210} \mathrm{Co}$-dicyanoazobenzene on $\mathrm{Au}(111),{ }^{211} \mathrm{Co}$-dicarbonitrilepolyphenyl on $\operatorname{Ag}(111),{ }^{212} \mathrm{Co}-4,4$ '-(ethyne-1,2-diyl)di-benzonitrile on $\mathrm{Ag}(111),{ }^{213} \mathrm{Cu}-1,3,8,10$ tetraazaperopyrene on $\mathrm{Cu}(111),{ }^{214,215} \mathrm{Cu}$-thiolate on $\mathrm{Cu}(111),{ }^{216} \mathrm{Mn}$-carboxylate on $\mathrm{Au}(111),{ }^{217}$ Au-cyanosexiphenyl on $\mathrm{Au}(111),{ }^{218}$ and the coordination between admolecules with alkali metals on $\mathrm{Au}(111),{ }^{219,220,221} \mathrm{~Pb}$-tetracyanonaphtho-quinodimethane on $\mathrm{Pb}(111),{ }^{222}$ and $\mathrm{Cs}$ atoms with aromatic carboxylic acids on $\mathrm{Cu}(100) .{ }^{171}$ Moreover, the formation of isolated organometallic chains on $\mathrm{Au}(111)$ was observed. The deposition of iodine-functionalized units (2',5'-diiodo-3,3",5,5'-tetramethyl-1,1':4',1'-terphenyl) on the metallic surface induces the release of iodine and allows bond formation between the molecule and diffusing gold adatoms. Long and flexible chains can thus be formed. ${ }^{223}$

\subsubsection{Image charge}

The image charge is a well-known method to model the electric field created by charges near a metal (conductive) surface. The system, consisting of a point charge $q$ located at a distance $z$ in a medium with a dielectric constant $\varepsilon_{1}$ above a flat metallic surface characterized by a dielectric constant $\varepsilon_{2}$, is equivalent to a system formed by the charge $q$ and another point charge $q$ ' at a distance of $2 z$ from $q$, such as $q^{\prime}=-\frac{\varepsilon_{2}-\varepsilon_{1}}{\varepsilon_{2}+\varepsilon_{1}} q .{ }^{224}$ The charge $q^{\prime}$ is the so-called image charge, and these two charges give rise to the so-called image-potential states. A molecule can be modeled as a distribution of net charges located on each atomic site, which allows the generalization of the charge image method by summing the various contributions of the system of charges. Recently, a novel method was developed by Golze et al. to include the polarization between a molecule and a metallic surface in hybrid quantum mechanics/molecular mechanics calculations. ${ }^{225}$ For 
instance, the image-charge contribution represents less than $10 \%$ of the adsorption energy for a thymine or a guanine molecule on $\mathrm{Au}(111)$, i.e., $0.96 \mathrm{kcal} / \mathrm{mol}(41 \mathrm{meV})$ and $1.41 \mathrm{kcal} / \mathrm{mol}(61$ $\mathrm{meV}$ ) for total adsorption energies of $14.89 \mathrm{kcal} / \mathrm{mol}(645 \mathrm{meV})$ and $17.06 \mathrm{kcal} / \mathrm{mol}(740 \mathrm{meV})$, respectively. Beyond the single-molecule case, this polarization effect has also been studied with increasing molecular coverage, such as benzene on $\mathrm{Cu}(111)^{194}$ or benzoate on $\mathrm{Cu}(110){ }^{226}$ exemplifying the role of the local molecular environment and thus the many-body character of this contribution.

\subsubsection{Crystalline faces}

The basic idea of treating a surface as a solid and inert support is often too simplistic a model; incorporating the surface structure generally requires a more refined view. This factor is important for metallic surfaces, of course, but even more so for semiconducting surfaces for which there is substantial reconstruction, giving rise to various adsorption properties. The different surface orientations offer not only different adsorption sites but also different electronic properties - the work function, for example. A prototypal example out of many is the codeposition of copper(II) tetraphenyl-21H,23H-porphirine (CuTPP) and cobalt(II) phthalocyanine $(\mathrm{CoPc})$. On an $\mathrm{Au}(111)$ surface, two phases are observed: a well-ordered one of CuTPP and a second disordered one comprising the two molecules, whereas on an $\mathrm{Au}(100)$ surface, a well-organized binary molecular array of CuTTP and CoPc is observed. ${ }^{227}$ In terms of adsorption sites, the molecules experience a larger adsorption energy on the tetragonal surface structure than on the hexagonal one, resulting in a higher mobility on the (111) geometry. Additionally, by replacing CuTPP by copper(II) 2,3,7,8,12,13,17,18-octaethyl-21H,23Hporphine (CuOEP), Yoshimoto et al. obtained organized structures on $\mathrm{Au}(111),{ }^{228}$ an observation 
that emphasizes the delicate balance between the intermolecular interaction and the diffusion barrier on the surface. For a chemisorbed system such as a full monolayer of 3,4,9,10-perylene tetracarboxylic di-anhydride (PTCDA) on $\operatorname{Ag}(111)$ and on $\operatorname{Ag}(110)$, Zou et al. identified, by combining various electronic and structural characterization techniques, noticeable changes of adsorption properties according to the surface structure. ${ }^{229}$ With a bicomponent system such as a $\mathrm{ZnPc}$ and $\mathrm{ZnOEP}$ bi-molecular array on $\mathrm{Au}(111)$ and $\mathrm{Au}(100)$ surfaces, Yoshimoto et al. demonstrated via STM experiments at the liquid/solid interface that an alternately arranged striped structure occurs on $\mathrm{Au}(100)$, indicating that the substrate provides less influence in terms of intermolecular interaction, whereas a supramolecular structure with a "chessboard" arrangement is observed on $\mathrm{Au}(111){ }^{230}$

\subsubsection{Comparison between adsorption on noble $\mathrm{Cu}, \mathrm{Ag}$, and Au surfaces}

It is generally assumed that the reactivity of noble metal surfaces follows the order of copper $>$ silver $>$ gold. ${ }^{155}$ For instance, $\mathrm{CuPc}$ molecules are physisorbed on $\mathrm{Au}(111)$ and are chemisorbed on $\mathrm{Cu}(111)$, with an intermediate case in terms of substrate bonding for $\operatorname{Ag}(111) .{ }^{231,232}$ Similarly, a single PTCDA molecule on (111) surfaces exhibits the same trend, with an increasing molecule/surface distance of $2.66 \AA, 2.86 \AA$, and $3.27 \AA$ for $\mathrm{Cu}, \mathrm{Ag}$, and $\mathrm{Au}$, respectively. ${ }^{233}$ Similar results have been obtained with diindoperylene $(2.51 \AA, 3.01 \AA$, and $3.10 \AA$, respectively). ${ }^{234}$ The distance between two neighboring metallic atoms on the (111) surface is $2.55 \AA, 2.89 \AA$, and $2.88 \AA$ for $\mathrm{Cu}, \mathrm{Ag}$, and $\mathrm{Au}$, respectively. This structural fact alone does not explain adsorption tendencies, especially for silver and gold, and electronic contributions, such as charge transfer, must to be taken into account. ${ }^{235}$ The electronic coupling between the molecule and the surface modifies the adsorption geometry, leading to a deformed admolecule, 
as was reported for $\mathrm{F}_{16} \mathrm{CuPc}{ }^{236,237,238}$ Moreover, a study by Heimel et al. has demonstrated the fundamental role of the surface nature on the reduction of the electronic gap of semiconducting molecules (pentacenequinone and pentacenetetrone). ${ }^{239}$ Once adsorbed, the oxygen atoms bend towards the surface, resulting in a deformation of the carbon skeleton and a modification of the metal-molecule interfacial electronic structure, to a dramatic level in the case of a copper surface.

Finally, the different adsorption behaviors of cyano-functionalized triarylamines on the three coinage surfaces should be highlighted. ${ }^{240}$ Whereas adsorption on $\mathrm{Au}(111)$ and $\mathrm{Ag}(111)$ produces well-ordered phases with dipolar coupling (H-bonding and metal coordination for the former and H-bonding only for the latter), adsorption on $\mathrm{Cu}(111)$ generates sparse 2D islands formed by metal coordination. The key role of the molecule/surface interaction for the formation of the bicomponent assembly is exemplified by the co-deposition of CuPc and PTCDA. ${ }^{241}$ The mixing is not favored, and separated islands are formed due to a stronger adsorption interaction of the two molecules on $\mathrm{Ag}(111)$ than for that of CuPc/PTCDA. The resulting system consists of large, densely packed CuPc domains condensed on a compact PTCDA monolayer on Ag(111), whereas one observes a highly ordered mixture of these two molecules on $\mathrm{Cu}(111){ }^{242}$

\subsubsection{Diffusion barriers}

As outlined above, the ability of molecules to move on a surface is one of the major factors when producing self-assembled structures. The adsorption energy provides information on how and whether molecules attach to a surface (in the direction perpendicular to this surface) and is distinct from the diffusion energy describing the mobility of an adsorbate on the substrate (in a direction parallel to the surface). ${ }^{243}$ The diffusion energy corresponds to the energy to transfer an adsorbate from one adsorption site to a neighboring one, e.g., from one hollow site across the saddle point of a bridge site on a [111] surface. In the case of low diffusion energy, adsorbates 
move freely on the surface and can be treated as a 2D gas phase. For instance, thioalkanes chemisorbed on a gold surface experience a low diffusion barrier once adsorbed. The strong covalent bond between the sulfur atom and the gold surface does not prevent high diffusion, giving rise to well-documented organosulfur/ $\mathrm{Au}(111)$ self-assembled monolayer systems. ${ }^{12,244}$ In contrast, when the bonding energy is of the same order of magnitude as the diffusion energy, the adsorbate behavior on the surface is more sensitive to temperature effects, and an equilibrium exists between a 2D gas phase on the surface and the gas phase itself. Although surface diffusion of atoms and of small molecules, such as carbon monoxide, is well understood (and often follows a simple single particle hopping model), the problem is significantly more complex in the case of larger molecules ${ }^{245}$ because they span several surface adsorption sites, ${ }^{246}$ deform during diffusion, ${ }^{247}$ interact with surface defects, ${ }^{248}$ can induce local surface reconstruction (see below), ${ }^{249}$ can diffuse only in specific directions, ${ }^{250}$ and can be immobilized or highly mobile depending on the orientation with respect to the surface atomic lattice, ${ }^{251}$ among other effects. Larger molecules are therefore more likely to be pinned (i.e., prevented from diffusing). However, to a first approximation, post-deposition annealing is in general sufficient to provide enough energy to allow diffusion of larger molecules.

In the case of coadsorption of two molecules, at intermediate temperatures and when the surface diffusion barriers of the two components are sufficiently different, it is possible that only one component is mobile. Such a scenario was recently employed by Amabilino, Kantorovitch, Raval et al. to design a bicomponent system comprising a molecular walker moving between immobilized molecular fences. ${ }^{252}$

\subsubsection{Molecule-induced surface reconstruction}


Finally, in certain cases, the presence of adsorbed molecules may lead to a reconstruction of the surface. For instance, it has been shown that adsorption of Azure A or pentacene (Pen) or trimethylphosphine modifies $\mathrm{Au}(111)$ herringbone reconstruction and eventually removes surface atoms and induces the formation of etch pits. ${ }^{253,254,255}$ In another example, Besenbacher et al. showed that hexa-tert-butyl-decacyclene molecules (HtBDC) deposited on $\mathrm{Cu}(110)$ surfaces create a characteristic trench under the molecule in which $14 \mathrm{Cu}$ atoms are removed from the surface layer in two neighboring close-packed rows. ${ }^{256}$ Similarly, the adsorption of a Lander molecule on $\mathrm{Cu}(211)$ leads to a reconstruction of the surface (311) steps. This effect of surface reconstruction induced by molecular adsorption was also observed at the step edge of a $\mathrm{Cu}(110)$ surface. In this example, metallic atoms diffusing along the lower terrace were trapped by molecules adsorbed on the upper step edge and modified the structure of the step by aggregation and molding. ${ }^{257}$ The observations of these surface structure modifications underneath a molecular adsorbate were obtained by STM manipulation at low temperature by displacing the molecules. Although we are not aware of such observations in the case of bicomponent self-assembled systems, it is likely that such a phenomenon exists and acts to locally perturb molecule-substrate interactions.

\subsubsection{Résumé of the various interactions responsible for molecular self-assembly}

The different ranges of interactions according to their strength are summarized, along with their directionality, in Table 1. Such a classification is difficult to establish due to the intricate character of some of these interactions. The range of the force strength varies generally by one, or sometimes two, orders of magnitude as a result of the diversity of molecular species encountered in the context of self-assembly.

\begin{tabular}{|l|l|l|l|}
\hline Interaction & Strength & Strength & Directionality \\
\hline
\end{tabular}




\begin{tabular}{|l|c|c|c|}
\hline & $(\mathrm{kcal} / \mathrm{mol})$ & $(\mathrm{eV})$ & \\
\hline $\mathrm{vdW}$ & $0.2-2.5$ & $0.01-0.1$ & No \\
\hline Substrate-mediated & $0.2-2$ & $0.01-0.1$ & No \\
\hline $\mathrm{CH}-\pi$ & $1-4$ & $0.05-0.2$ & Yes \\
\hline$\pi-\pi$ bond & $0.2-11.5$ & $0.01-0.5$ & Partially \\
\hline X-bond & $2.4-36$ & $0.1-1.5$ & Yes \\
\hline H-bond & $1-16$ & $0.05-0.7$ & Yes \\
\hline Dipole-dipole & $1-11.5$ & $0.05-0.5$ & Yes \\
\hline Cation- $\pi$ & $1-18$ & $0.05-0.8$ & Yes \\
\hline Image charge & $0.2-2.5$ & $0.01-0.1$ & Yes \\
\hline Electrostatic & $1-70$ & $0.05-3$ & No \\
\hline Ion-dipole & $11.5-46$ & $0.5-2$ & Yes \\
\hline $\begin{array}{l}\text { Metal } \\
\text { complexation }\end{array}$ & $11.5-70$ & $0.5-3$ & Yes \\
\hline Covalent & $23-100$ & $1-4$ & Yes \\
\hline
\end{tabular}

Table 1. Strengths of molecular interactions. Values extracted from the literature and adapted from Barth, J. V. ${ }^{56}$

\section{SELF-ASSEMBLY BY DIRECTIONAL INTERACTIONS: COMPLEMENTARY H- BONDING MOIETIES}

As discussed above, the most widely employed non-covalent interaction in supramolecular chemistry is H-bonding, ${ }^{258}$ which allows control over the directionality and strength of interactions when both components are carefully designed. By co-adsorbing two types of molecules with single (or even more with multiple) complementary H-donors (D) and Hacceptor groups (A), the intermolecular interaction can be reinforced. Thus, it is possible to reduce the respective role of the interactions with the substrate, which often governs the orientation of the supramolecular arrangement. ${ }^{259}$ The relative importance of the substrate 
generally increases with decreasing intermolecular H-bond strength. Fig. 1 shows typical functional groups often encountered in the literature.
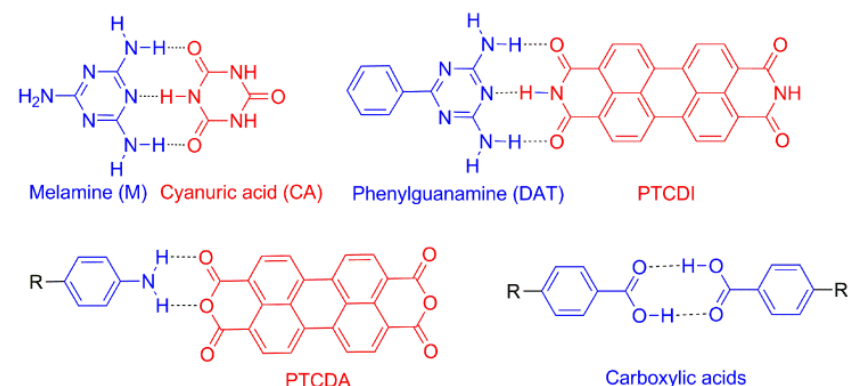

Figure 1. Examples of different combinations of molecules containing hydrogen-donor (D) or hydrogen-acceptor (A) functional groups for H-bonded networks: DAD: Melamine (M, 2,4,6-triamino-1,3,5-triazine), guanamine (or 2,4-diamino-1,3,5-triazine, DAT) derivatives; ADA: cyanuric acid (CA, 1,3,5-triazine-2,4,6-triol), AA: PTCDA, carboxylic acid-pyridine, AD: carboxylic acids.

\subsection{Melamine-based structures}

The melamine molecule, simultaneously acting as an H-bond donor by its three amino groups and an H-bond acceptor by its three triazine nitrogen atoms, is of special interest in engineering 2D bicomponent nanostructures. Its small size and high symmetry make it an ideal model for advanced theoretical analyses and comparative experiments to understand the geometry and the formation mechanisms of supramolecular structures. Melamine alone can form homomolecular self-assembled monolayers on $\mathrm{Au}(111),{ }^{260,261,262,263} \mathrm{Ag}-\mathrm{Si}(111),{ }^{264}$ and $\mathrm{Ag}(111) .{ }^{265} \mathrm{On} \mathrm{Au}(111)$, melamine can assemble in two possible arrangements: a hexagonal structure and a close-packed structure. Theoretical studies ${ }^{261,266}$ including vdW interactions have suggested that the closedpacked regions are transition regions (or domain walls) between the more stable hexagonal islands. Another theoretical analysis ${ }^{267}$ indicated that the formation mechanism relies on molecule-metal atom pairs that participate in the assembly growth via a specific gating 
mechanism mediated by cooperative molecular interactions. It is suggested that this formation mechanism is not restricted to the melamine gold system. On $\operatorname{Ag}(111),{ }^{265}$ melamine forms two different structures: first, a metastable honeycomb structure stabilized by three double H-bonds per molecule, which is similar to the one observed on $\mathrm{Au}(111)$ or $\mathrm{Ag}-\mathrm{Si}(111)$; then, annealing of this phase leads to the thermodynamically stable phase, exhibiting a higher packing density and an additional H-bond between the amino groups of adjacent molecules. Melamine has been used to prepare monolayers of bi-molecular systems with other molecular blocks bearing hydrogendonor and acceptor groups, such as cyanuric acid (CA), PTCDI, PTCDA, 1,4,5,8-naphthalenetetracarboxylic di-imide (NTCDI).

\subsubsection{Melamine and cyanuric acid}

The 1:1 H-bonded adduct between CA and melamine (M) that forms rosettes has long been considered an outstanding case of non-covalent synthesis. ${ }^{268,269,270}$ In 2006, a self-assembled monolayer CA-M directed by lateral triple H-bonding interactions was prepared through two different methods in UHV on $\mathrm{Ag}-\mathrm{Si}(111) \sqrt{ } 3 \times \sqrt{3} R 30^{\circ}$ : sequential deposition of CA followed by $\mathrm{M}$ (the reverse order did not produce any useful results) and co-deposition of the molecular species. ${ }^{271}$ The results were in both cases a structure composed of $3 \mathrm{M}$ and $3 \mathrm{CA}$ molecules that formed a 2D array of cyclic hexamers (Fig. 2). 


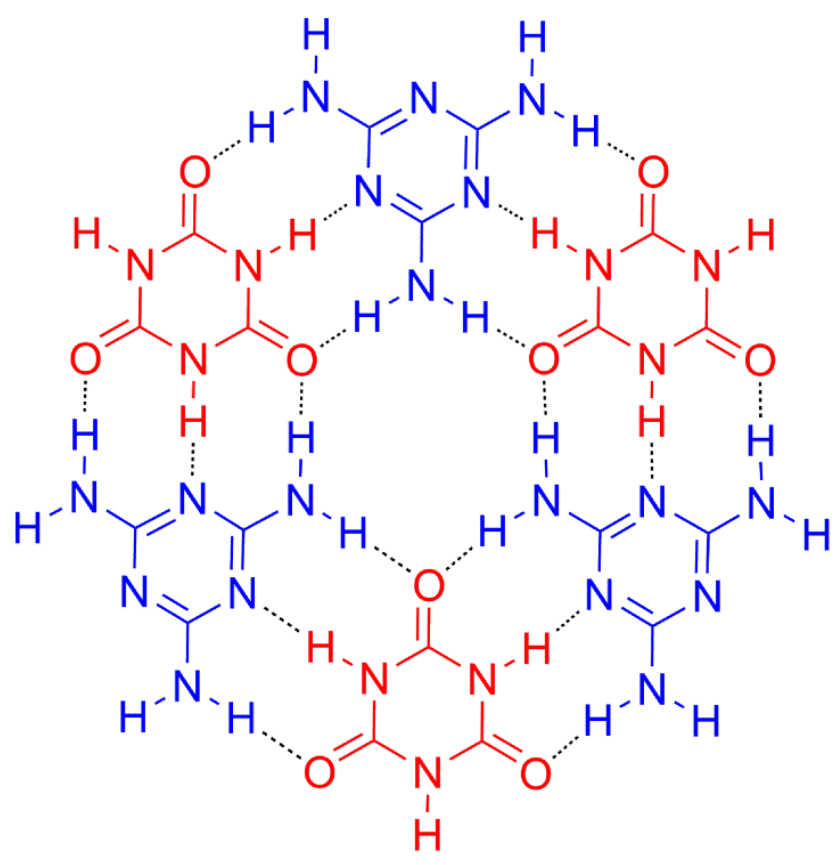

Figure 2. Hexameric motif constituting the CA-M binary network stabilized by H-bonding.

Interestingly, sublimation of $\mathrm{M}$ on $\mathrm{CA}$ ordered-layer islands led to a honeycomb structure at the edges of the CA domains, attributed to the formation of CA-M complexes. After leaving these coexisting islands overnight, the CA desorbed, leaving only the more stable complex islands. Simultaneous deposition produced the complex directly without traces of single component islands. Both methods resulted in networks uncontaminated by the precursor phases due to the higher stability of the bicomponent network. The authors observed a subtle change in the lattices due to molecule-substrate interactions that led the network to relax in the preferred orientation.

In 2008, a similar study was conducted on $\mathrm{Au}(111)$ by Besenbacher et al.. ${ }^{272}$ Both deposition techniques were used on this substrate. This time, the molecular units M or CA were deposited separately, and networks of $\mathrm{M}$ or CA were imaged; then, the second component was deposited to allow the formation of large heteromolecular networks. The H-bond lengths of 1.86 $\AA(\mathrm{O} \cdots \mathrm{H})$ and $1.96 \AA\left(\mathrm{N}^{\cdots} \mathrm{H}\right)$ were in good agreement with those obtained by bulk X-ray diffraction (1.93 $\AA$ and $2.01 \AA$, respectively), indicating the molecule-substrate interactions to be 
negligible. ${ }^{262}$ In addition, theoretical results confirmed that the lattice parameters were consistent with measured values comprising a binding energy of $0.82 \mathrm{eV}(18.91 \mathrm{kcal} / \mathrm{mol})$ per molecule for the stoichiometric network. By carrying out the sequential deposition of $\mathrm{M}$ followed by $\mathrm{CA}$, the authors could also produce the new stable phase $\mathrm{CA}_{1} \mathrm{M}_{3}$. A binding energy of $0.68 \mathrm{eV}(15.68$ $\mathrm{kcal} / \mathrm{mol}$ ) per molecule was calculated for this phase, of comparable magnitude to the $0.65 \mathrm{eV}$ $(14.99 \mathrm{kcal} / \mathrm{mol})$ per molecule of the pure CA network. It was proposed that this $\mathrm{CA}_{1} \mathrm{M}_{3}$ is an intermediate step in the incorporation of $\mathrm{CA}$ into an existing $\mathrm{M}$ network towards the thermodynamically stable $\mathrm{CA}_{1} \mathrm{M}_{1}$ binary mixture phase. A third superstructure has also been observed in which hexagonal rings of $\mathrm{M}$ are interlinked by $\mathrm{CA}$ molecules showing a chiral arrangement.

A similar $\mathrm{CA}_{1} \mathrm{M}_{1}$ hydrogen-bonded network has been observed on thin films of $\mathrm{NaCl}$ on $\mathrm{Au}(111),{ }^{273}$ which indicates that this stable self-assembly is not significantly affected by the isolating $\mathrm{NaCl}$ substrate.

\subsubsection{Melamine and dicarboxylic imides}

Similar to CA, the diimides NTCDI or PTCDI have been key precursors for the elaboration of bicomponent architectures. ${ }^{274,275}$ These compounds are photochemically stable dyes whose derivatives are considered promising new materials for organic electronics. They include two diformylamine $(-\mathrm{C}=\mathrm{O}-\mathrm{N}(\mathrm{H})-\mathrm{C}=\mathrm{O}-)$ hydrogen acceptor-donor-acceptor units linked through an extended aromatic backbone. PTCDI or NTCDI have been deposited alone on several surfaces $\left[\mathrm{NaCl}(001),{ }^{276} \mathrm{Au}(111),{ }^{277,278,279} \mathrm{Ag} / \mathrm{Si}(111),{ }^{264,280}\right.$ or layered substrates $\left.{ }^{281}\right]$, and various phases have been observed originating from H-bonding between molecules. 
In 2003, Beton et al. reported ${ }^{282}$ the controlled deposition of M and PTCDI in UHV on the silver-terminated silicon surface $\mathrm{Ag}-\operatorname{Si}(111) \sqrt{3} \times \sqrt{ } 3 R 30^{\circ}$. They obtained a honeycomb arrangement at RT that exhibited three strong H-bonds per M-PTCDI pair (Fig. 3).
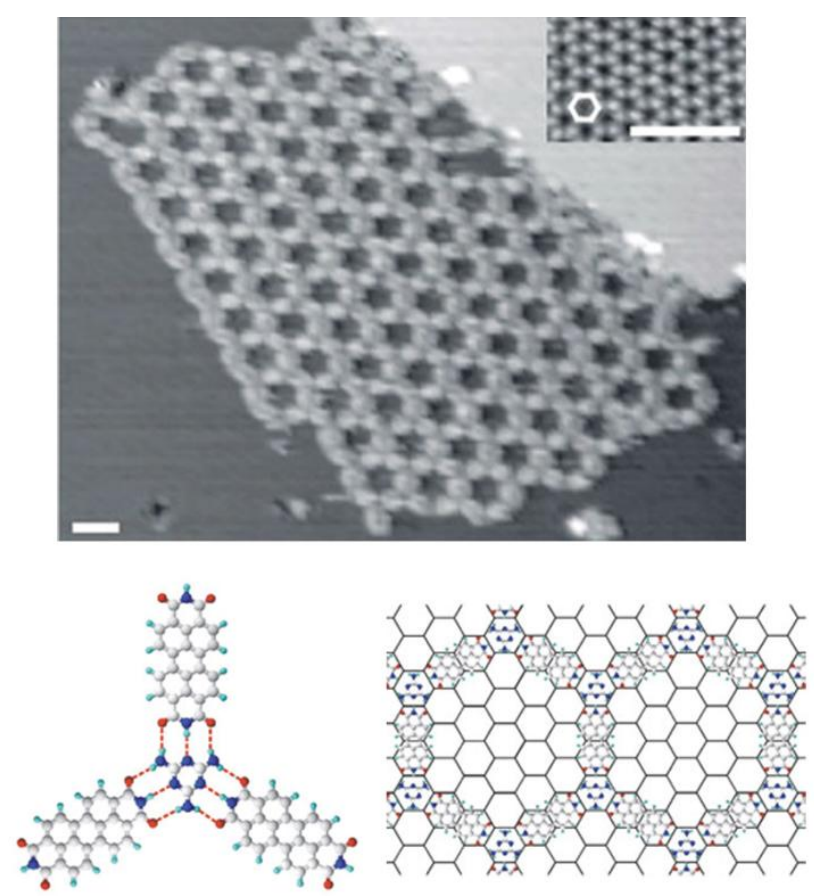

Figure 3. Top: STM image of the PTCDI-M network. Scale bar: $5 \mathrm{~nm}$. Bottom: PTCDI-M H-bond arrangement showing the registry with the substrate honeycomb network. Reprinted with permission from Ref. ${ }^{282}$. Copyright 2015 Nature publishing group.

Later, in 2007, same authors reported an analogous study on $\mathrm{Au}(111)-(22 \times \sqrt{3})$ : after sequential deposition of PTCDI and $\mathrm{M}$ and subsequent annealing at $60-80^{\circ} \mathrm{C}$, hexagonal networks were reported. ${ }^{283}$ Annealing at $\sim 90^{\circ} \mathrm{C}$ produced a new parallelogram arrangement. The co-existence of both phases is possible if subjected to an intermediate annealing. The parallelogram arrangements (Fig. 4) are chiral and more compact than the hexagonal ones. Within the rows, one end is stabilized by a PTCDI-PTCDI double H-bond plus a single one with M; the other PTCDI extremity forms a triple H-bond with M. To summarize, annealing and surface-mediated 
effects are clearly outlined in these reports, generating in particular a new parallelogram arrangement on $\mathrm{Au}(111)$.
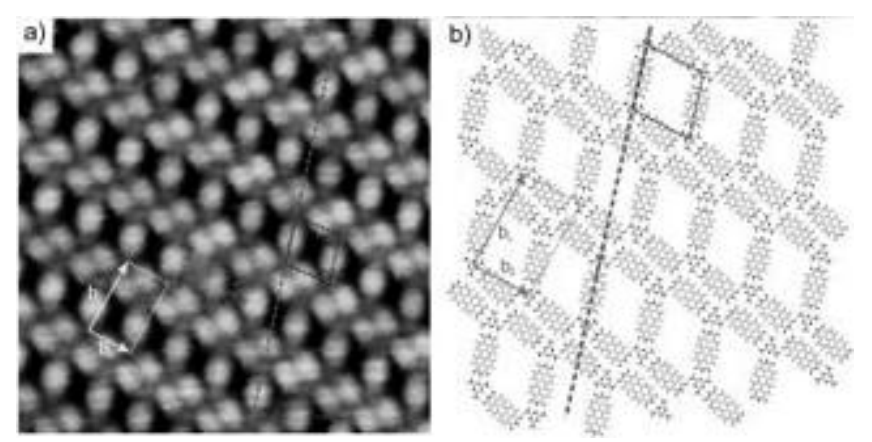

Figure 4. (a) Parallelogram motif of the PTCDI-M (ratio 3:2) binary network stabilized by H-bonding on Au(111) after annealing at $90^{\circ} \mathrm{C}$ : unit cell vectors $b_{1}$ and $b_{2}$ are measured at $30.7 \pm 0.4 \AA$ and $19.4 \pm 0.3 \AA$, respectively, with an internal angle of $87 \pm 2^{\circ}$; (b) schematic representation of the networks with the different parameters of the cell, where the pore is depicted by a dashed box. Reprinted with permission from Ref. ${ }^{283}$. Copyright 2015 Wiley.

Interestingly, Silly et al. have worked on the same system but with a different PTCDI-M ratio (3:4), affording a new close-packed chiral "pinwheel" structure as imaged by STM (Fig. 5). ${ }^{278}$ After an extensive study of the single molecular species, the authors suggested that the codeposition of the new ratio at RT leads to a novel superstructure composed of six internal M molecules as a hexagonal center surrounded by PTCDI pairs. The entire nanodomain is stabilized by H-bonding, and both chiral forms are observed. Two notable features are the $\mathrm{M}$ hexagon being rotated by $\sim 15^{\circ}$ compared to the unimolecular network and this center domain also containing molecular defects. This example nicely demonstrates how a new parameter can significantly tune a molecular arrangement to exhibit pronounced complexity. 


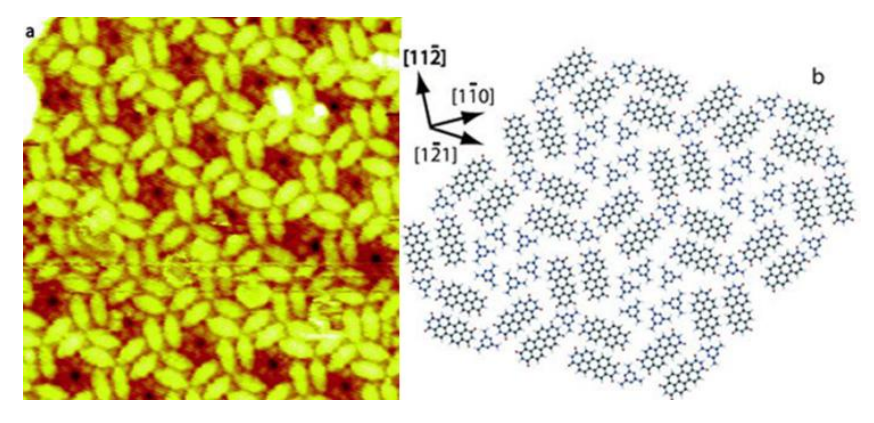

Figure 5. (a) One chiral nanodomain composed of PTCDI-M (ratio 3:4) on Au(111) as imaged by STM, the M hexagon showing low resolution due to possible internal rotations or defects; (b) Model of the molecular arrangement detailed above. Reprinted with permission from Ref. ${ }^{278}$. Copyright 2015 RSC.

The same authors introduced another way to tailor the geometry of the PTCDI-M long-range arrangement using a reconstructed $\mathrm{Au}(111)-(22 \times \sqrt{3})$ surface (grown on mica). ${ }^{284}$ After deposition of the two precursors and annealing at $80^{\circ} \mathrm{C}$ for $15 \mathrm{~h}$, the network is composed of a succession of double rows of hexagons and a single row of parallelograms (see Fig. 6).

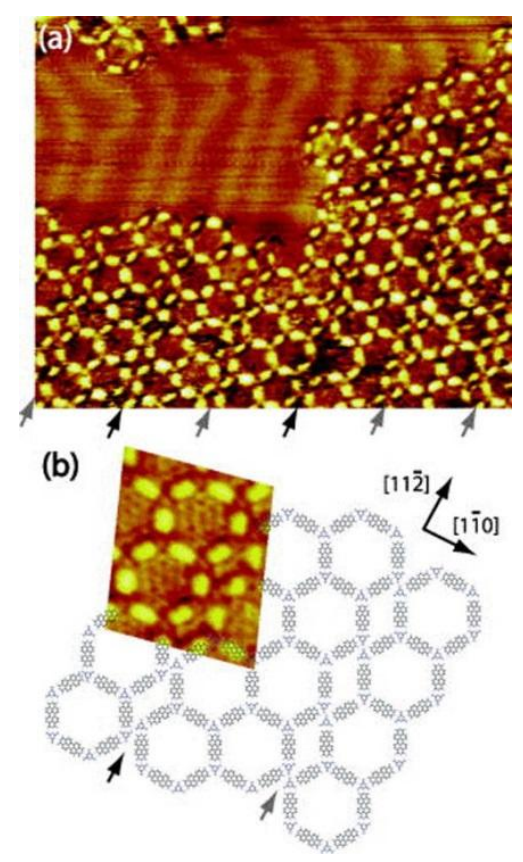

Figure 6. (a) STM image of the supramolecular network of the epitaxial PTCDI-melamine supramolecular network on the reconstructed $A u(111)-(22 \times \sqrt{ } 3)$ surface. (b) Molecular model with a superimposed high-resolution STM 
image. Black and gray arrows indicate the two types of parallelogram rows. Reprinted with permission from Ref. 284. Copyright 2015 AIP.

These motifs are $\sim 6.3 \mathrm{~nm}$ apart, fitting the periodicity of the $\mathrm{Au}(111)-(22 \times \sqrt{3})$ substrate in the [1-10] direction. A sub-substrate interaction in epitaxy was proposed to explain this complexity of interactions resulting in such a structure. A first-principles study of these bicomponent films on $\operatorname{Ag}(111)$ surface was conducted ${ }^{285}$ and revealed strong distortions of the adsorbed molecules near the contact points due to the influence of the H-bonds. The calculated charge transfer from the substrate to the PTCDI molecule $\left(0.9 \mathrm{e}^{-}\right)$has an influence on the H-bonds by inducing a redistribution of the electronic s-cloud. Although it weakens $\mathrm{N}^{\cdots \cdots} \mathrm{H}-\mathrm{N}$ bonds and strengthens the two $\mathrm{O} \cdots \mathrm{H}-\mathrm{N}$ bonds, these dipolar effects are in balance. Another study used functionalized PTCDI to induce nanoporous arrays of various geometries and symmetries. ${ }^{286} \mathrm{Di}$ (propylthiol)PTCDI exhibited two distinctive arrangements when adsorbed on the surface as a close-packed array and a honeycomb structure with pores, respectively. Modification with M (deposition time of $4 \mathrm{~h}$ with a sample held at $60-80^{\circ} \mathrm{C}$ ) resulted in a hexagonal structure with expanded pore size: this technique represents a notable example of sequential development of self-assembled structures through increasing the pore dimensions by associating di(propylthiol)-PTCDI with M. $\mathrm{Br}_{2}$-PTCDI is also reported to produce a porous network with $\mathrm{M}$. The authors highlighted that through chemical modification, in situ functionalization of $\mathrm{Br}_{2}$-PTCDI can offer more possibilities to tailor the pore properties and geometry. Several other minority phases with more complex intermolecular junctions were also observed on $\mathrm{Au}(111)$, exhibiting various pore sizes amenable to filling with $\mathrm{C}_{60}$ molecules. ${ }^{260}$

Similarly, co-adsorption of $\mathrm{M}$ plus NTCDI was investigated by Beton et al. on Ag$\operatorname{Si}(111) \sqrt{3} \times \sqrt{3} R 30^{\circ}$ under UHV. The aim was to study the level of control by replacing one 
component by a similar one possessing the same triple hydrogen-bond system. ${ }^{264}$ The array of molecules studied in this paper is expected to give results analogous to PTCDI but with smaller unit cell vectors; however, experimental results have revealed more complex motifs, which displayed commensurability with the surface. To afford a wider coverage of the mixed phase, after primary deposition of NTCDI, M was sublimated onto the surface held at $60-80^{\circ} \mathrm{C}$ although M-M and NTCDI-NTCDI arrangements remained abundant. High-resolution imaging was possible, and three characteristic binary structures, in strong contrast to the one observed with PTCDI, were identified: triangular, star and melamine tape as a consequence of M-M bonding (not observed with PTCDI, except on $\mathrm{Au}(111)$ under certain conditions ${ }^{264}$ ). High commensurability between adsorbates and the substrate provides strong evidence of the important role played by the surface in the global stabilization of the final assemblies. Typically, a surface-mediated effect is probably the source of low intermolecular H-bond interactions in this case, avoiding the creation of the expected long-range bi-unit modules. Once again, the active role of the surface should be emphasized in this example (Fig. 7).
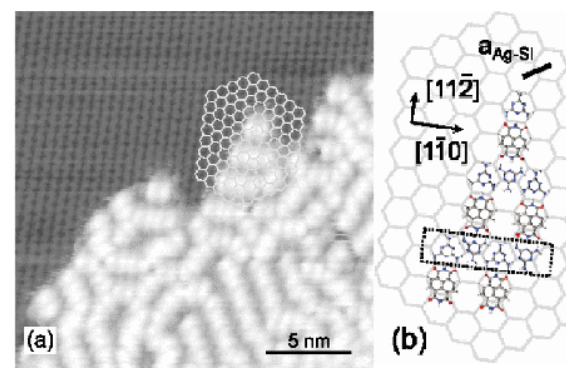

(b)

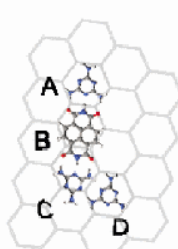

(c)

Figure 7. NTCDI-M on $\mathrm{Ag}-\mathrm{Si}(111) \sqrt{ } 3 \times \sqrt{ } 3 \mathrm{R} 30^{\circ}$. a) Upper left side corner: hexagonal mesh of the reconstructed surface. b) and c) Models of the structure. A row of $M$ maintained by H-bonds is highlighted in the dashed rectangle. Reprinted with permission from Ref. ${ }^{264}$. Copyright 2015 APS.

\subsubsection{Melamine and PTCDA}

Similar to PTCDI, perylene tetracarboxylic di-anhydride (PTCDA) (see Fig. 1) is a much- 
investigated prototype molecule for organic electronics. ${ }^{287}$ It has been extensively studied $^{288,289,290}$ and reviewed ${ }^{233}$ as a homolayer on different metallic surfaces. It has been suggested that PTDCA/Ag(111) interactions are mainly driven by chemisorption (with the carboxylic $\mathrm{O}$ atoms positioned $0.18 \pm 0.03 \AA$ closer to the surface than the perylene core), ${ }^{291}$ while PTDCA is physisorbed on $\mathrm{Au}(111)$. In contrast, with the acceptor-donor-acceptor system of bis-imides, the PTCDA system includes two hydrogen-acceptor AAA anhydride moieties ($\mathrm{O}=\mathrm{C}-\mathrm{O}-\mathrm{C}=\mathrm{O}-)$.

Coadsorption of PTCDA at sub-monolayer coverage with melamine ${ }^{292}$ on Ag$\operatorname{Si}(111) \sqrt{ } 3 \times \sqrt{ } 3 R 30^{\circ}$ leads to two distinct stable phases: a hexagonal lattice and a double-row structure completely different from the structure of PTCDA alone. ${ }^{293}$ M's donor-acceptor-donor (DAD) coupling motif is not well suited to the AAA interface offered by PTCDA. However, STM allows clear visualization of hexagons (in general 30 units) with both period and molecular orientations similar to those of PTCDI-M, as previously described. The two $\mathrm{N}-\mathrm{H} \cdots \mathrm{O}$ bonds can overcome the repulsive character between the central anhydride oxygen atoms and the triazine ring nitrogen. The interaction energy was calculated to be $0.13 \mathrm{eV}$ (3 kcal/mol) per PTCDA-M couple, ${ }^{292}$ corroborating the model proposed by the authors with weak interaction. Another closepacked phase (Fig. 8) consists of an alternating double row of PTCDA and M alongside the hexagonal network. In contrast, the same blend of molecules on $\operatorname{Au}(111)(22 \times \sqrt{3})$ leads to the formation of several networks, depending on the PTCDA-M ratio, with a clear intermixing of molecules in the superstructures. ${ }^{294,295}$ 


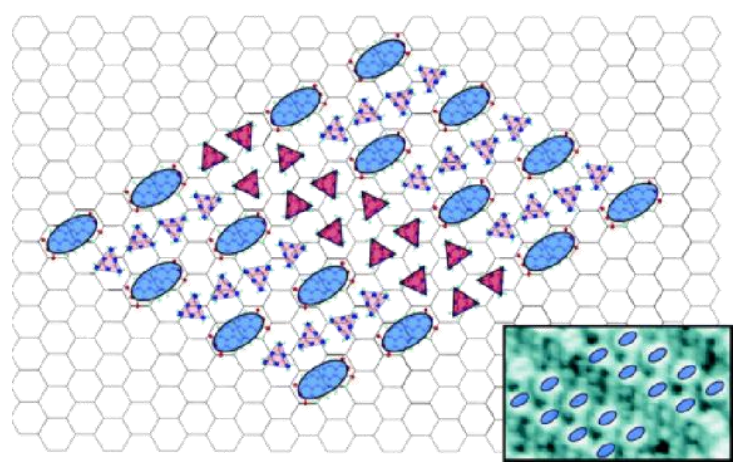

Figure 8. PTCDA molecules are highlighted with ovals, and melamine molecules are shown as triangles, shaded darker for the melamine double row, which is more easily resolved in the images. Inset: STM image. Reprinted with permission from Ref. ${ }^{292}$. Copyright 2015 ACS.

\subsection{Other dicarboxylic imide/anhydride/acid-based self-assembled systems 3.2.1 PTCDI or NTCDI and guanamine derivatives}

Guanamine and 2,4-diamino-1,3,5-triazine (DAT) derivatives are molecules analogous to melamine in which the functionalization in the position 6 of the triazine ring allows a larger number of structures, in particular linear ones, with H-bonding systems similar to those described with melamine. 


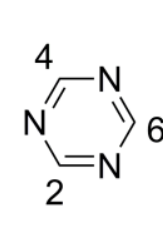

Triazine<smiles>Nc1nc(N)nc(N)n1</smiles>

Melamine<smiles>Nc1nc(N)nc(-c2ccc(-c3nc(N)nc(N)n3)cc2)n1</smiles>

Phenyldiguanamine or BDATB<smiles>Nc1nc(N)nc(-c2ccccc2)n1</smiles>

PhDAT

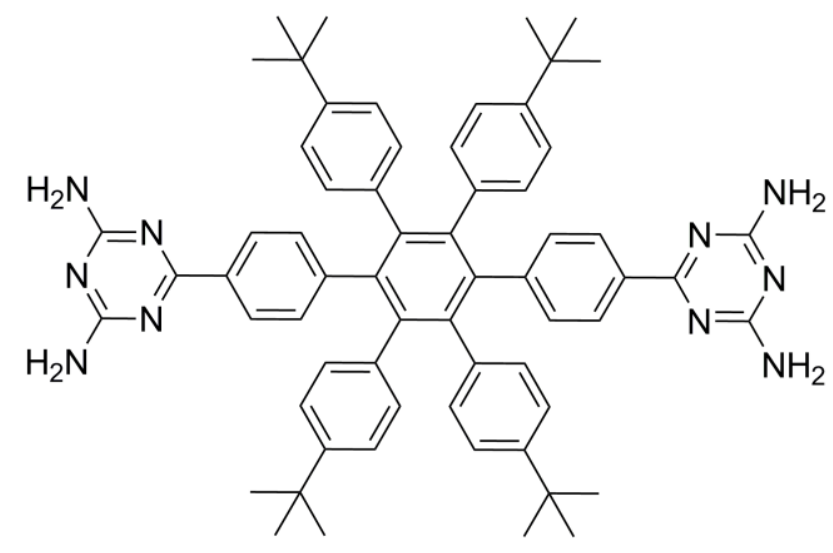

Lander DAT

Figure 9. Diaminotriazine derivatives involved in bicomponent self-assembled systems.

The first example of uniaxial anisotropy using phenyldiguanamine (BDATB) was obtained by co-evaporation of NTCDI on gold surfaces. ${ }^{296}$ First, on flat Au(111) surfaces, a 2D binary supramolecular structure was formed by packing 1D heteromolecular NTCDI-BDATB rows. Theoretical calculations showed that the triple H-bonds inside the rows $(14.4 \mathrm{kcal} / \mathrm{mol}, 0.62 \mathrm{eV})$ are much stronger than lateral binding between adjacent dimers $(4.6 \mathrm{kcal} / \mathrm{mol}, 0.2 \mathrm{eV})$, therefore defining a highly anisotropic supramolecular phase. Then, to test a pre-patterning-plus-selfassembly approach of bicomponent structures, a molecular monolayer was grown on an $\mathrm{Au}(111)$ vicinal surface with periodic faceting, namely, $\mathrm{Au}(455)$ (Figure 10). 


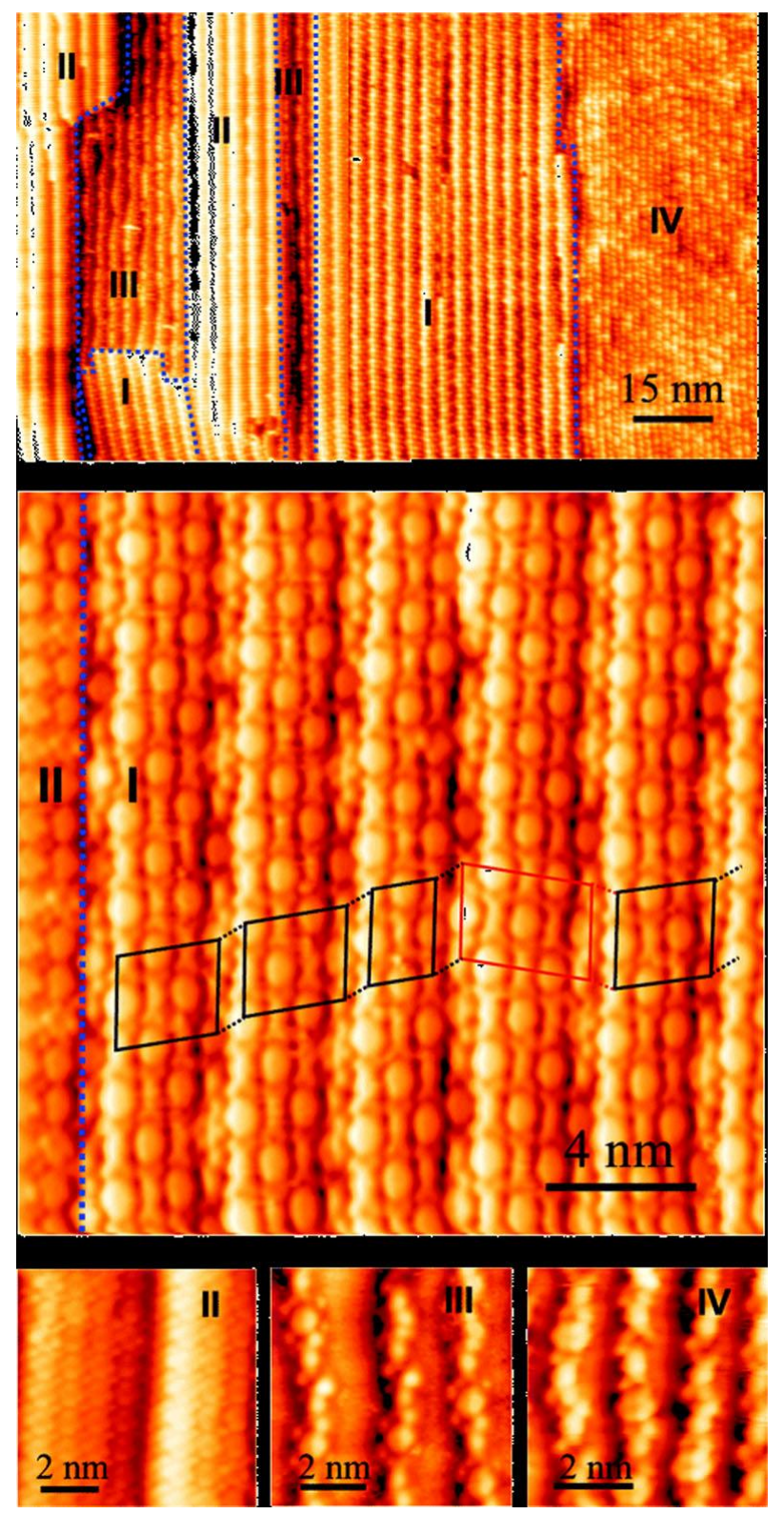

Figure 10. Coadsorption of 0.9 monolayer (ML) of NTCDI and benzodiguanamine (BDG) on Au(455). A $1 D$ multiple-phase separation (dotted blue lines) is observed. The binary, single domain molecular recognition structure lined up along step edges (phase I) is shown in the central panel. Black rhomboids mark the regular packing sequence of $1 D$ chains on terraces, which is reversed in one of them (red rhomboid). The bottom panels show the detailed view of phases II (NTCDI), III (BDG-rich on wide terraces), and IV (BDG-rich on narrow terraces). Reprinted with permission from Ref. ${ }^{297}$. Copyright 2015 ACS. 
A comparison with pure phases of NTCDI and BDATB on the latter substrate showed that the binary chains are forced along the vicinal steps as expected but also that the ideal bicomponent structure was limited to surfaces with relatively large terraces. Steps induce a distinct hierarchy of driving forces that can lead to multiple phase segregations. The spectroscopic fingerprints of these monolayer assemblies on $\mathrm{Au}(111)$ have been obtained by XPS, ultraviolet photoemission, STS and NEXAFS and have provided a precise description of the electronic states of the $\mathrm{N}$ and $\mathrm{O}$ species of the amine and imide functional groups that give rise to networks with a binary mixture. ${ }^{298,299}$ This work illustrated the bipolar charge redistribution induced by the charge polarization in these anisotropic H-bonded systems. Moreover, the molecule-substrate interactions are weak for each of the single component layers and for the bicomponent one. However, the strong H-bonding in the bicomponent layer affects mainly the electronic structure of the s orbitals due to a modification of the bond lengths. Next, Fasel, Müllen et al. ${ }^{277}$ published similar results with BDATB and PTCDI on vicinal $\mathrm{Au}(11,11,12)$. Here, also, the growth of the specific 1D H-bonding network was guided by the appropriate template surface. It was also shown that single rows contained more defects than double rows, with a larger number of homomolecular pairs; it was concluded that the presence of a second row stabilizes the ordering by lateral adsorbate-adsorbate interactions. Similarly, the Hbonding fingerprints in electronic states were obtained by STM/STS experiments ${ }^{300}$ and confirmed these results.

An in-depth analysis of interplay and hierarchy of interactions in the analogous systems PTCDIBDATB/6-phenyl-DAT/6-(4'-cyanophenyl)-DAT has also been published by Fasel et al. ${ }^{301}$ 


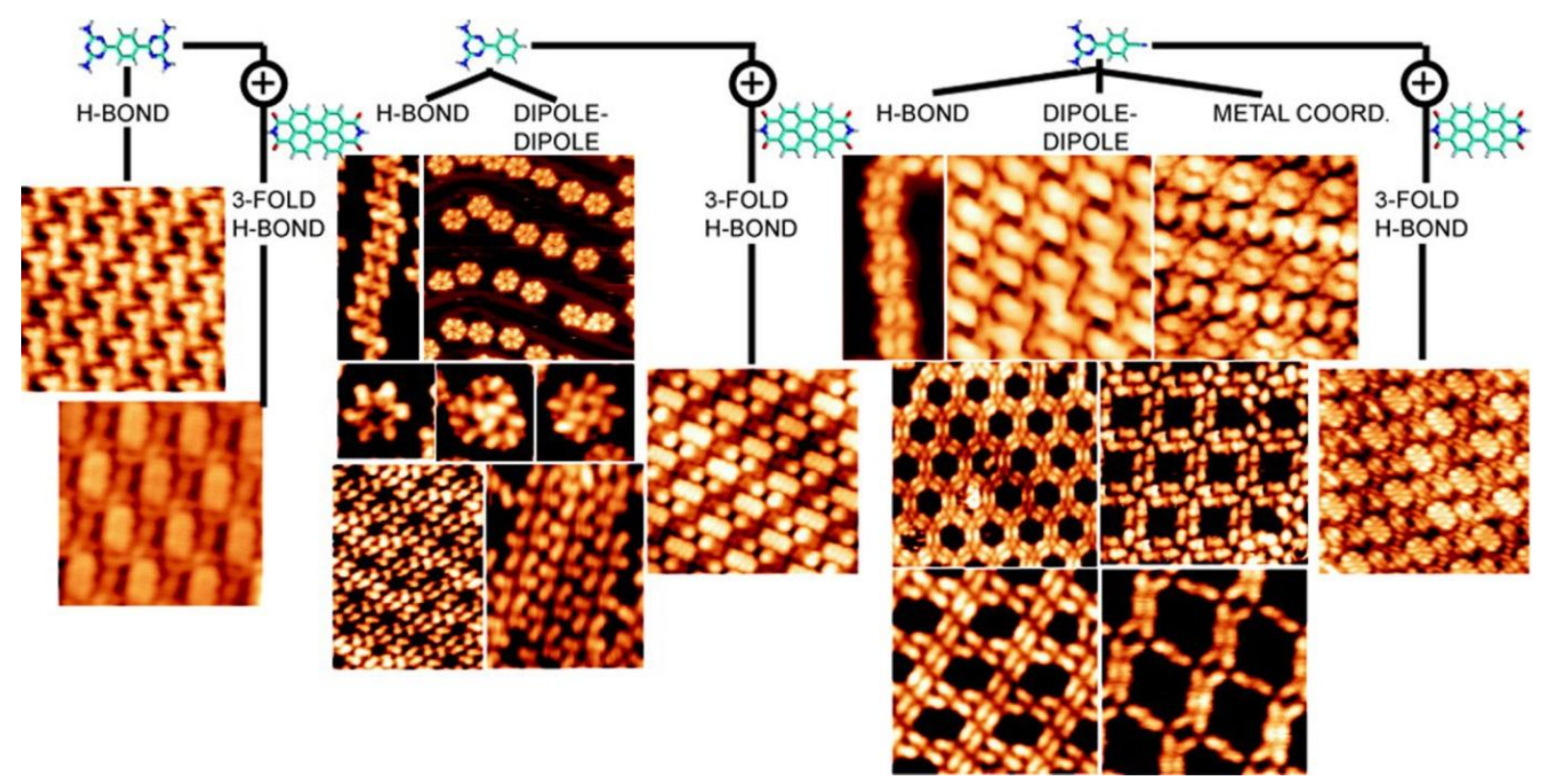

Figure 11. Mono- and bicomponent self-assembly of BDATB, 6-phenyl-DAT (PhDAT), 6-(4'-cyanophenyl)-DAT (CPhDAT) (see Fig. 9) and PTCDI. Reprinted with permission from Ref. ${ }^{301}$. Copyright 2015 ACS.

As shown in Fig. 11, each of these molecules comprises one (two in the case of BDATB) guanamine D-A-D group, and, in the case of CPhDAT, a cyano group that can be involved in metal-organic bonds. The comparison between the behaviors of these molecular species allowed the author to explore first in homomolecular systems, then in binary systems with PTCDI, the interplay and competition of H-bonding, dipole-dipole interactions and metal coordination. The presence of the complementary H-bonding units promoted the growth of single, well-ordered phases in comparison with homomolecular phases.

Notably, these mixed PTCDI-BDATB layers cannot be grown on ultra-thin $\mathrm{NaCl}$ films on $\mathrm{Au}(111)$ but are stable on $\mathrm{NaCl} / \mathrm{Au}(11,12,12) .{ }^{302}$ On a partly NaCl-covered surface, the layers grow preferentially on the first molecular adlayer on $\mathrm{Au}(111)$ rather than on $\mathrm{NaCl}$, indicating that the intermolecular interaction is stronger than the interaction with $\mathrm{NaCl}$, as is frequently 
observed for insulating surfaces. However, in contrast to what is observed on gold vicinal surfaces, the superlattice of bi-molecular wires does not form on thin films of $\mathrm{NaCl}$ / $\mathrm{Au}(11,12,12)$, and the small molecular domains do not follow the Au step directions but instead the $\mathrm{NaCl}(001)$ high symmetry directions. Actually, the distortion of the $\mathrm{NaCl}$ thin films induced by the underlying substrate modifies their electronic structure and generates a superlattice of binding sites on these insulating films.

This study was also extended to another template surface, the strain-relief pattern formed by two monolayers of $\mathrm{Ag}$ on $\operatorname{Pt}(111) .{ }^{303}$ It was shown that this template could also be used to control the orientation of the H-bonded rows by strong molecular pinning on the pattern.

In another example, large molecules equipped with two diamino-triazine and two dicarboxylic imide functionalities gave rise to 2D and 1D structures on $\mathrm{Au}(111){ }^{304}$ Two Lander molecules with diamino triazine (DAT) and dicarboxylic imide (DCI) groups show a 3D structure due to the presence of bulky spacer groups on the poly-aromatic board, which act as legs. In consequence, the direct H-bonding is altered by the conformational flexibility, allowing a stronger vdW contribution with the metallic surface (Fig. 12). This effect is reinforced when molecules are on step edges of the surface: short 1D heteromolecular chains are formed via complementary DAT-DCI triple H-bonds due to the ability of the DAT groups to rotate with respect to the central core of the Lander DAT to optimize the H-bonding. It was shown that this effect of adaptation of the DAT group by rotation is responsible for various supramolecular architectures not achievable with planar compounds. ${ }^{305}$ 

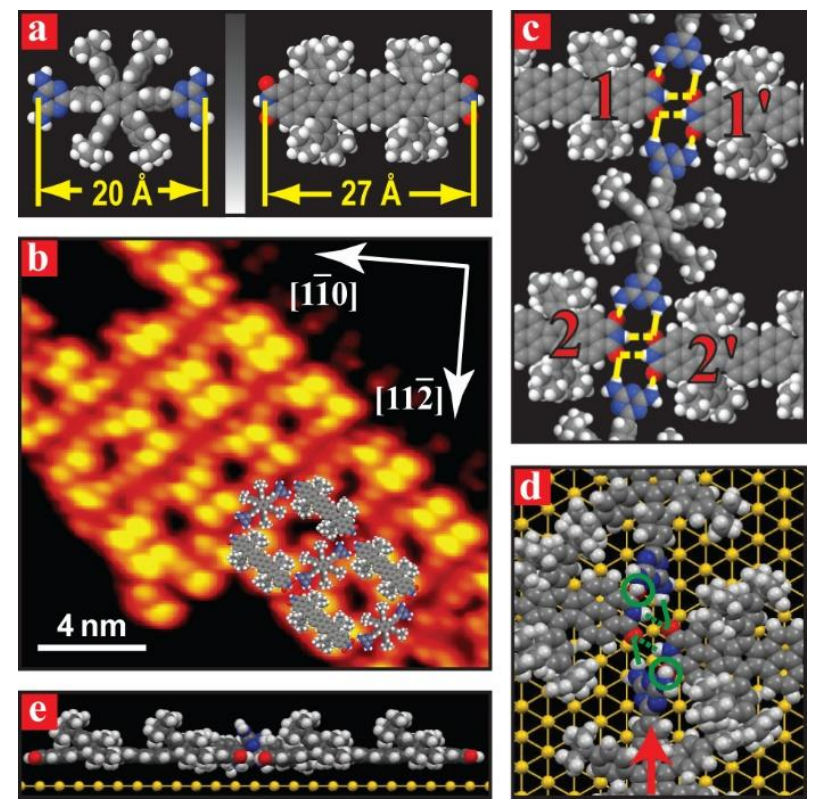

Figure 12 (a) Two Lander molecules with DAT and DCI functionalities. (b) STM image of 2D grid-like pattern and (c) a model of this structure. (d) top-and (e) side-views of the calculated model showing the H-bonds involved in the formation of the $2 D$ network. The arrow in (d) indicates the viewing direction for the side view. Reprinted with permission from Ref. ${ }^{304}$. Copyright 2014 RSC.

\subsubsection{PTCDA and amines}

Another example involving PTCDA in a bicomponent phase has been obtained by co-adsorption with the hydrogen donor AA-AA 1,2,4,5-tetraaminobenzene (TAB) on Ag/Si(111), ${ }^{306}$ in which PTCDA and TAB form alternating rows stabilized by H-bonding between a dianhydride and two $\alpha$-amine groups. The experiment was performed by first depositing a PTCDA layer. After subsequent deposition of TAB (requiring several minutes), some of the previous islands were converted into parallel rows with alternated brightness as a new intermixed phase. The complete transformation was obtained after an overnight deposition of TAB. Interpretation based on the lattice of the silver-terminated silicon surface underneath allows precise determination of the spatial arrangement: the row spacing corresponds to three times the lattice constant $\mathrm{a}_{0}$ in the [1- 
10] direction (with $\mathrm{a}_{0}=6.65 \AA$ ). These results are supported by DFT geometry optimization calculations and confirm the presence of H-bond supramolecular interactions between anhydride and amine. The complete conversion of all PTCDA domains to this mixed row phase, which is not the case, for instance, for PTCDI-melamine despite a stronger triple $\mathrm{H}$-bond, is a consequence of the weaker homomolecular PTCDA-PTCDA interaction strength compared to the PTCDI-PTCDI one. In the case of PTCDA, heteromolecular PTCDA-melamine interactions enter the competition and favor the mixed phase.

PTCDA has also been associated with 4,4"-diamino-p-terphenyl (DATP) or 2,4,6-tris(4aminophenyl)-1,3,5-triazine (TAPT) on $\mathrm{Au}(111)$ in detailed theoretical ${ }^{307}$ and experimental studies. ${ }^{308}$
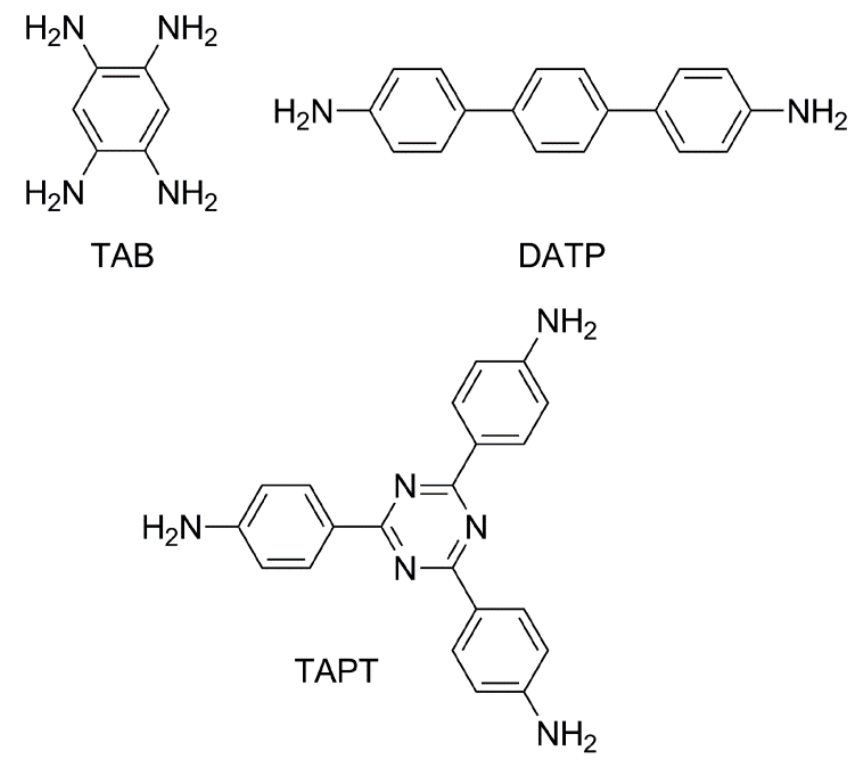

Figure 13. Benzene-1,2,4,5-tetraamine (TAB), [1,1':4',1"-terphenyl]-4,4"-diamine (DATP) and 4,4',4"-(1,3,5triazine-2,4,6-triyl)trianiline (TAPT).

Submonolayers of PTCDA deposited with DATP form an open rectangular H-bonded 1:1 network stabilized by four $\mathrm{NH}^{\cdots} \mathrm{O}$ bonds at each corner. The authors regard this network as an array of regularly spaced, identically oriented PTCDA aligned with the $\langle 110\rangle$ directions of the 
substrate and, orthogonal to PTCDA, the long axis of DATP lying along the $\langle 211\rangle$ directions. At higher coverage, different superstructures were observed in which pairs of DATP aligned along the $<211>$ directions separate single or double rows of PTCDA. In contrast, with the bicomponent layers discussed so far, these superstructures depend crucially on both homo- and heteromolecular interactions, and some phases could be described as nanounits of PTCDA separated by DATP spacers. A detailed theoretical analysis of the electronic and geometrical structures of this system has shown that vdW-corrected DFT describes more precisely the interactions involved in such supramolecular layers on the metallic surface compared to standard DFT schemes without a vdW term. ${ }^{307}$

In contrast, coadsorption of PTCDA with TAPT produced one of the largest superstructures observed so far, with a unit cell containing seven PTCDA molecules grouped in a nanoisland surrounded by four TAPT molecules. 

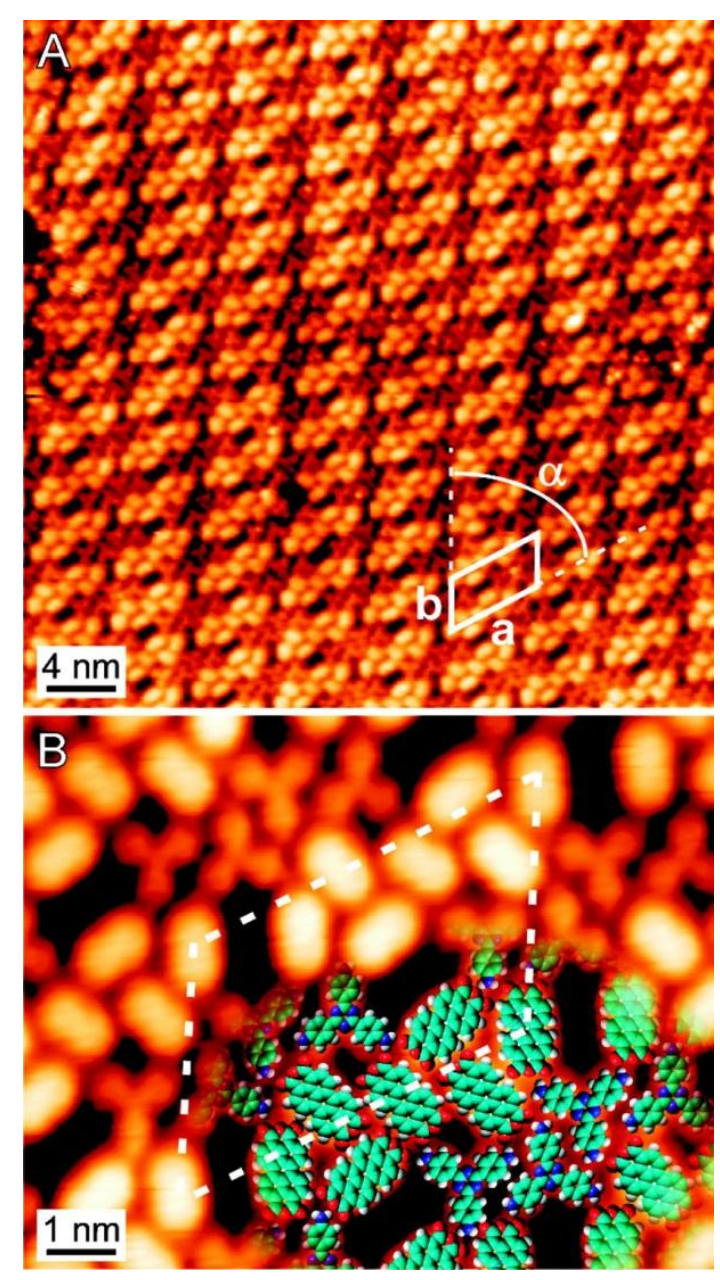

Figure 14. Overview (A) and detailed STM image with model (B) of array of seven-molecule PTCDA nanoislands produced using TAPT as a H-bonding spacer. Reprinted with permission from Ref. ${ }^{308}$. Copyright 2015 ACS.

The five central molecules in PTCDA islands show an ordering similar to the one observed in the pure PTCDA herringbone phase.

In summary, the H-bonds between PTCDA and these aromatic (mono)amines are in competition with PTCDA-PTCDA interaction due to their comparable bonding energies, which are significantly higher than that of the amine-amine ones. As a result of this balance, it is possible to obtain a variety of phases consisting of homomolecular nanoislands embedded into a matrix of the second species.

\section{Adenine and PTCDA}


In contrast, the coadsorption of symmetric PTCDA and the non-symmetric adenine (9- $H$-purin6-amine) DNA base on $\mathrm{Au}(111)$ leads to a complex bicomponent chiral supramolecular network comprising 10 molecules of PTCDA and 4 molecules of adenine. ${ }^{309}$ This network is composed of rows of PTCDA molecules connected by O...H-C H-bonds (along the red dashed line in Fig. 15.c), forming a large parallelogram pore. At the center of this structure, four PTCDA molecules self-assemble in a domino-like manner. The four adenine molecules fill the voids left between these two arrangements, but with some randomness and chirality within each void. It is noteworthy that this complex packing is the only one observed with this pair of molecules. DFT calculations have been performed (without including the presence of the substrate) to understand the stability of this network. These calculations indicated that the presence of adenine provides significant strengthening to the PTCDA network.

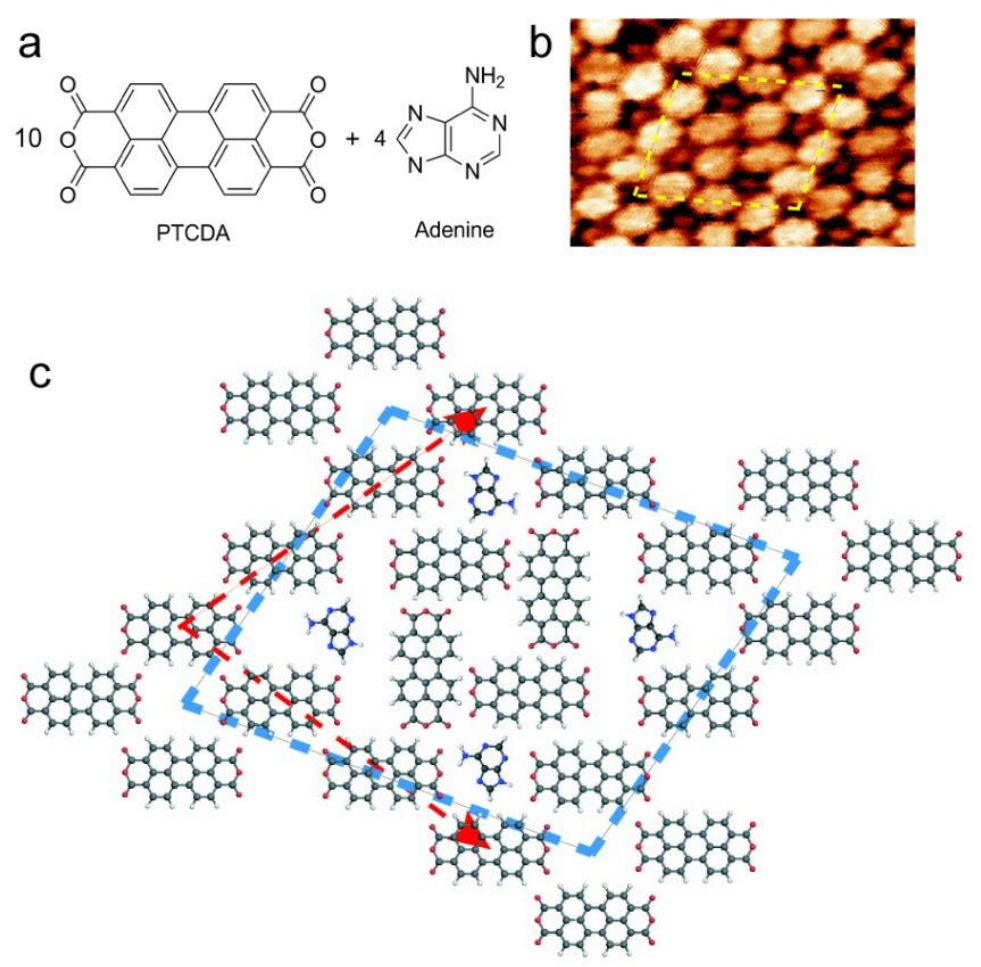


Figure 15. a-b) Coadsorption of PTCDA and adenine on Au(111)-(22 $\times \sqrt{3})$ in the ratio of 10:4. The unit cell is indicated by dashed yellow lines. c) Model of the bicomponent packing. The outer skeleton of PTCDA is underlined by the red dashed red lines. Adapted with permission from Ref. ${ }^{309}$. Copyright 2016 ACS.

\subsubsection{Carboxylic acids}

Carboxylic acids self-assemble by double H-bond interactions as exemplified in the case of trimesic acid, Fig. 16. To form bimolecular networks, the second molecule must be able to establish a bond with the carboxylic group that is strong enough to compete with the acid-acid bond, as in the case of the basic pyridyl group.

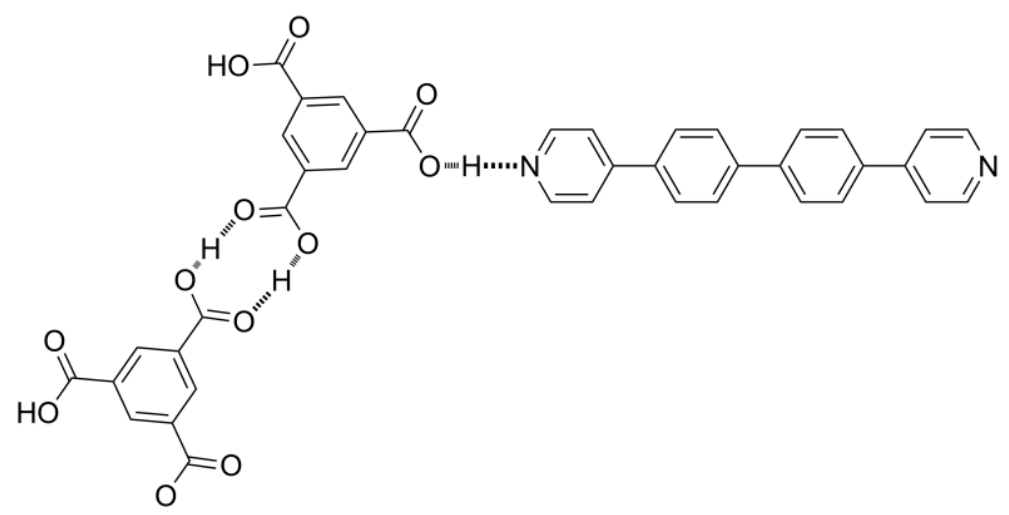

Figure 16. Trimesic acid (TMA) and basic 4,4'-bis(4-pyridyl)-biphenyl (BPBP) and possible homo (acid-acid) or heteromolecular (acid-base) H-bonds.

TMA, being a carboxylic acid, is prone to self-assemble by acid-acid H-bonding such that the homomolecular clusters of TMA compete with the acid-base binary phases. Experimentally, ${ }^{310}$ two phases with a 1:1 ratio of TMA and BPBP (Fig. 16) were observed: a closed-packed herringbone structure and a rectangular porous one. Using these two phases as precursors, the authors developed two strategies to modify the TMA:BPBP ratios (Fig. 17). The first strategy was to deposit additional BPBP onto the substrate in a step-wise manner to obtain ratios below unity, i.e., with an excess of BPBP. The second strategy was to increase the substrate temperature, causing the stepwise desorption of BPBP and giving rise to TMA:BPBP ratios 
greater than unity. As a result, six distinct pore shapes (rectangular, triangular, diamond, pentagonal and hexagonal) were observed. A unified structural model was developed to rationalize the observed structures and predict as-yet-unobserved ones, in particular using the concept of "framework knot units", the TMA oligomers or clusters connected by BPBPs.

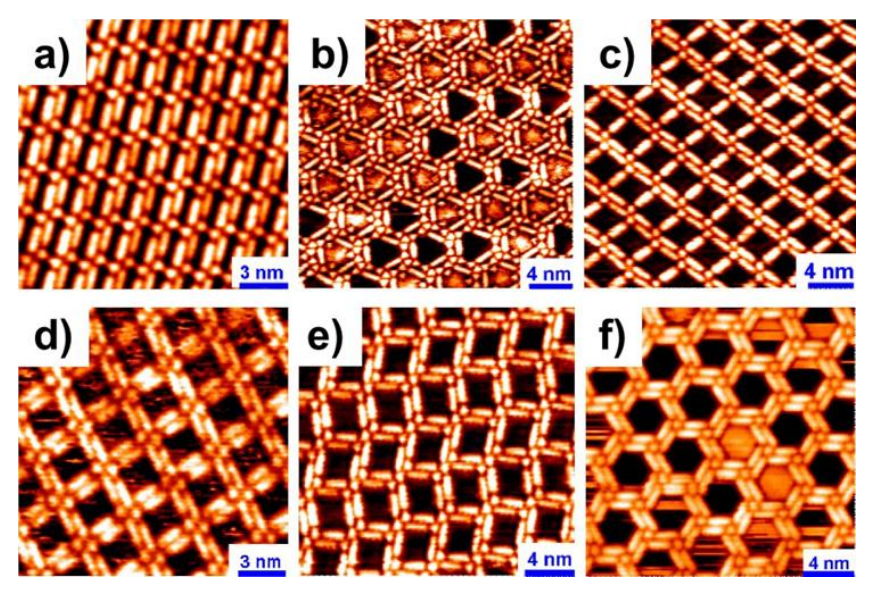

Figure 17. Images of a) rectangular pattern with TMA:BPBP ratio of $1: 1$; b) with a ratio of $8: 3$; c)-f) with ratios of less than unity. Reprinted with permission from Prof. $K . W u$.

Another example of bimolecular networks based in carboxylic acids is the self-assembly of croconic acid interacting with 3-hydroxyphenalenone; both are organic ferroelectrics, resulting in the formation of $2 \mathrm{D}$ features as a function of the stoichiometry and showing a tendency to aggregate tetrameric building blocks. ${ }^{311}$

\subsection{Biology-related compounds}

Efforts in supramolecular engineering have been guided by nature on many occasions; thus, it is not surprising that natural compounds such as amino-acids or nucleobases have been studied on noble metal surfaces. ${ }^{312,313,314,315,134,135}$

For instance, the four nucleobases cytosine $(C),{ }^{316}$ guanine $(G),{ }^{317}$ adenine $(A d),{ }^{103}$ and thymine $(\mathrm{T})^{318}$ form well-organized $1 \mathrm{D}$ and $2 \mathrm{D}$ structures on $\mathrm{Cu}, \mathrm{Ag}$ and $\mathrm{Au}$ surfaces. The $\mathrm{H}-$ 
bonding in these homomolecular systems is sufficiently strong that the role of the surface can be idealized as a simple support with minimal influence over the self-assembly process.

Binary nucleobase sets of complementary $\mathrm{G}+\mathrm{C}$ and non-complementary $\mathrm{Ad}+\mathrm{C}$ were studied by Besenbacher et al. on the inert $\mathrm{Au}(111)$ surface in UHV conditions. In 2008, they first reported $^{319}$ that both pairs can be formed after step-by-step deposition at RT. Heating created a significant difference in the stability of the hydrogen-bonding of the two systems: after heating at $373 \mathrm{~K}$ for $10 \mathrm{~min}$, the $\mathrm{C}+\mathrm{G}$ binary systems remained almost unaltered; after $10 \mathrm{~min}$ at $353 \mathrm{~K}$, the C+Ad mixture appeared as segregated islands of pure C or Ad. Such findings were obtained in the absence of water or a phosphate backbone, thus revealing that the triggered recognition of base pairing probably does not require the presence of a scaffold. It has been proposed that planar surfaces serving as a reservoir for a $2 \mathrm{D}$ gas of base molecules and triggering recognition between complementary bases could have played an active role in the first steps of the origin of life. A similar experiment with a mixture of complementary Ad and T nucleobases did not produce any conclusive results, as it was difficult to analyze the images. In 2010, the same group developed a strategy for the formation of a porous supramolecular network on the same noble metal surface by molecular recognition based on $N$-aryl-modified G and C nucleobases. ${ }^{320}$ These new complementary units may be viewed as mimics of nucleobases when attached to DNA. First, deposition of each base led to well-ordered chains maintained by H-bonding. The interacting phenyl rings and the methyl groups of the neighboring molecules to form the $2 \mathrm{D}$ supramolecular array provided weak lateral interchain binding. Sequential deposition (C, then G, at RT before imaging at a lower temperature) led to a highly ordered supramolecular bicomponent architecture with a flat porous network (Fig. 18). DFT calculations and STM image simulations confirmed these experimental results. These findings can be seen as preludes to more 
sophisticated modifications towards the understanding of molecular recognition in base-pairing events.

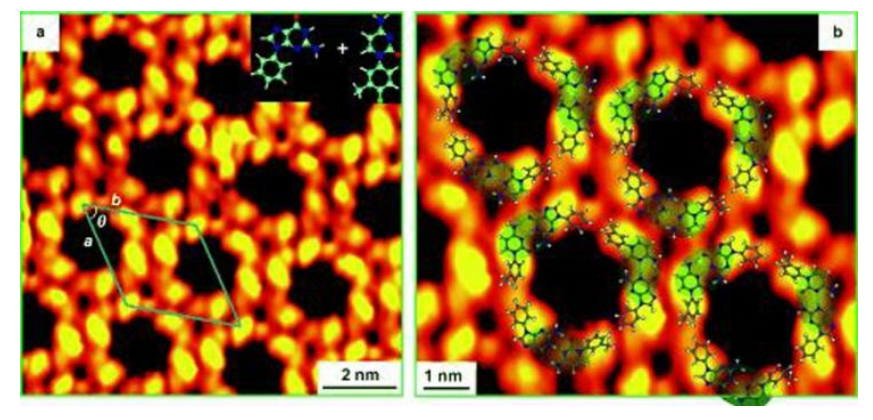

Figure 18. Binary mixture self-assembly of modified $C+G$ after sequential co-deposition: a) high-resolution image and b) DFT-calculated molecular model superimposed on the magnified image. Reprinted with permission from Ref. ${ }^{320}$. Copyright 2015 Wiley.

Along these lines, dipeptide chain nanostructures ${ }^{321}$ were studied by $2 \mathrm{D}$ co-crystallization between di-L-phenylalanine (L-Phe-L-Phe) with an organic linker terephthalic acid (TPA). Pure dipeptide did not form any continuous networks, producing only isolated chains characterized by a high density of kinks and defects, mainly due to non-perfect matching with the substrate and substrate-mediated repulsion between the chains. In contrast, through co-deposition of the TPA linker, large, homogeneous and highly periodic arrangements could be formed on anisotropic $\mathrm{Cu}(110)$ and isotropic $\mathrm{Cu}(100)$ (see Fig. 19). The TPA linker molecule does not affect the final chiral character of the $1 \mathrm{D}$ arrangement. For all of these reasons, this approach is probably sufficiently robust for general applicability and could be extended to a larger range of biomolecules for potential heterogeneous catalytic systems. 


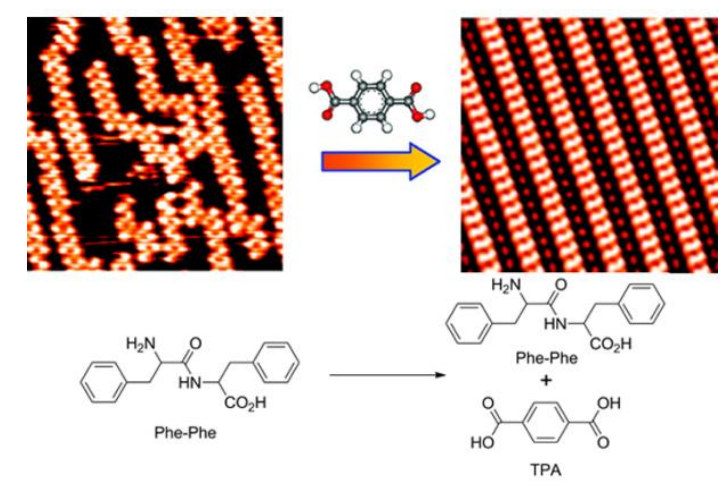

Figure 19: Effect of the TPA organic linker on the self-assembly process of L-Phe-L-Phe dipeptide (brighter spots) on Cu(110). Adapted with permission from Ref. ${ }^{321}$. Copyright 2015 ACS.

Finally, we review the successive deposition of amino acid L-methionine and tetracene on $\operatorname{Ag}(111) .{ }^{322}$ First, the L-methionine produces long and regular rows due to $\mathrm{O} \cdots \mathrm{H}$ interaction and generates a nanograting with a distance of $3.8 \mathrm{~nm}$ between the trenches. This inter-row distance can be tuned $(27.5 \mathrm{~nm}, 19.0 \mathrm{~nm}, 9.4 \mathrm{~nm}, 8.5 \mathrm{~nm}$, or $4.4 \mathrm{~nm})$ by adjusting the methionine concentration. ${ }^{134}$ This area offers a track for guest molecules such as tetracene molecules, which are confined in one dimension. These guest molecules do not decorate the methionine rims but instead stay surprisingly near the centers of the trenches.

The opportunity to integrate natural compounds into molecular devices is a significant achievement towards more sophisticated matters. However, more efforts in this direction should follow, as the library for potential devices is enormous (e.g., in surface-supported heterogeneous catalysis), provided that the problem of transfer in UHV of larger fragile and reactive molecules can be completely solved (e.g., by electrospray techniques coupled to soft-landing in UHV). ${ }^{36}$

\section{METAL-LIGAND COORDINATION}

Metal-ligand bonding is another elegant way of organizing supramolecular networks at the vacuum-solid interface. ${ }^{59}$ On-surface coordination chemistry has developed considerably in the 
past few decades, in particular thanks to the Kern, Barth, and Ruben groups as well as other groups working in the field. So far, most of the architectures have involved only one type of ligand, and bicomponent systems, which require significantly different chemical reactivities, have only been recently reported. Surface-confined bicomponent metal-organic chemistry relies on the interplay between more parameters than for H-bonded systems: different mobility of the adsorbates, i.e., a) metal adatoms and ligands, b) chemical reactions of the ligand with the surface, which can change its reactivity, and c) modification of the metal center properties by the metallic substrate, among other factors. One of the key parameters is the important role of the substrate, which acts as a template to direct and stabilize the metal-organic framework. ${ }^{323,324}$ The importance of surface template effects was illustrated in a study of dumbbell-shaped supramolecules obtained by heteroleptic coordination of copper atoms by the pyridyl and bipyridyl nitrogen atoms of bis(4-pyridyl)-1,4-benzene (BPB) and (2,2'-bipyridine)-5,5'dicarboxylic acid (BDA). ${ }^{325}$ Codeposition of these two ligands on a $\mathrm{Cu}(100)$ surface at $\mathrm{RT}$, followed by annealing at $400 \mathrm{~K}$, produced a bi-nuclear dumbbell-shaped complex $\mathrm{Cu}_{2}(\mathrm{BPB})(\mathrm{DBA})_{2}$ (Fig. 20).

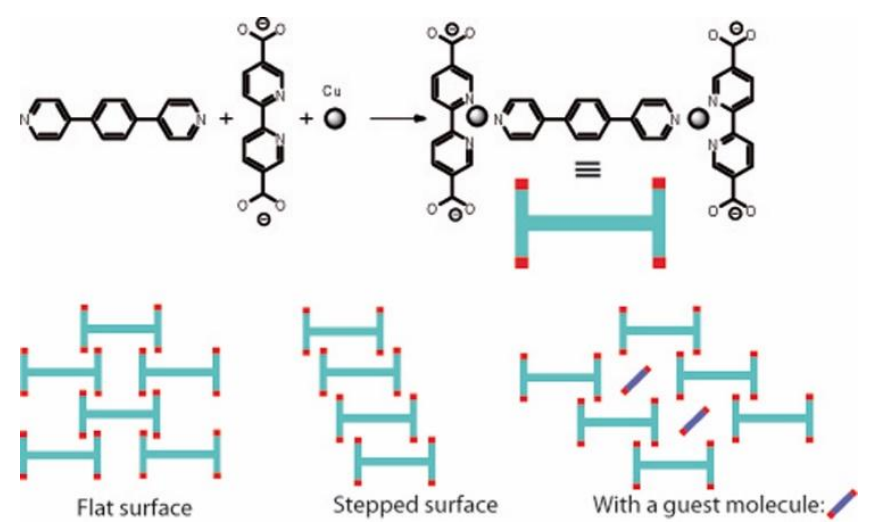

Figure 20. Supramolecular organization of dumbbell-shape complexes from bis(4-pyridyl)-1,4-benzene (BPB), (2,2'-bipyridine)-5,5'-dicarboxylic acid (BDA) and copper on Cu(100). 
Under these experimental conditions, the carboxylic groups are deprotonated by the surface (shown as red ends in Fig. 20) and are good hydrogen-bond acceptors. On defect-free terraces, the complexes are interconnected through multiple H-bonds between these carboxylate groups and the hydrogen atoms of the pyridyl groups, leading to a close-packed array with a brick-wall pattern. Near monoatomic step edges, the steric confinement induced by the step modifies the energy landscape of this H-bond network and favors a more linear organization that follows the orientation of the steps. Deposition of excess BDA produces a new structure in which the brick-wall pattern accommodates additional molecules without destroying the dumbbell complex.

An example of transition from a stable homoleptic 2D coordination layer to a $1 \mathrm{D}$ heteroleptic coordination network was demonstrated by Kern et al. in $2009 .{ }^{326}$ In the presence of $\mathrm{Cu}$ atoms, 4,4'-bis(4-pyridyl)(2,2'-bipyrimidine (PBP) (Fig. 21) self-organizes on $\mathrm{Cu}(100), \mathrm{Ag}(100)$ and $\operatorname{Ag}(111)$ surfaces $^{324}$ to form thermally stable and extended 2D metal-organic open pore coordination networks in which each copper atom is bonded to one pyridyl and two pyrimidyl nitrogen atoms.

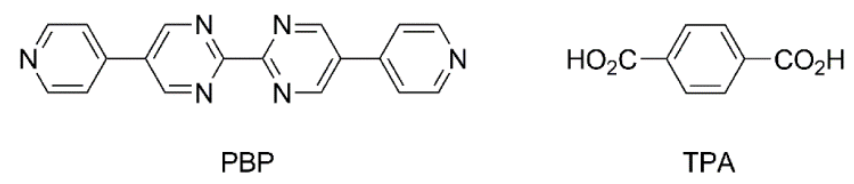

Figure 21. 4,4'-bis(4-pyridyl)2,2'-bipyrimidine (PBP) and terephthalic acid (TPA).

Subsequent deposition of TPA (Fig. 21) and annealing at $400 \mathrm{~K}$ resulted in highly ordered and extended domains of a mixed phase of both molecules. In this structure, the PBP molecules form linear Cu-pyridyl coordination chains interconnected by TPA molecules (Fig. 22). 


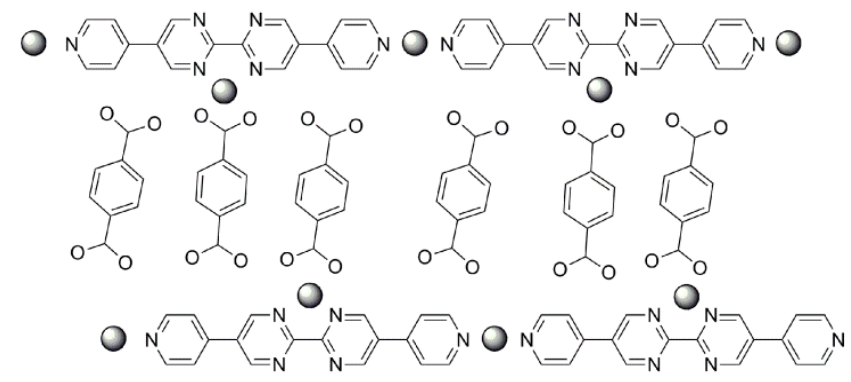

Figure 22. Tentative molecular model of the PBP-TPA mixed phase.

In this geometry, some of the carboxylate groups complete the coordination sphere around the copper atoms linked to the bipyrimidine nitrogen atoms, whereas the others maintain chain separation by H-bonds with the PBP hydrogen atoms. To sum up, the TPA molecules passivate the pyrimidine nitrogen atoms and prevent the growth of a $2 \mathrm{D}$ homotropic coordination network of Cu-PBP.

In contrast, the favored coordination geometry around an Fe atom with carboxylate groups and 4-pyridine groups (in absence of pyrimidine ligand) on a $\mathrm{Cu}(100)$ surface is represented by the $\left[\mathrm{Fe}_{2}(\text { carboxylate })_{2}(\text { pyridyl })_{2}\right]$ units shown in Fig. 23.

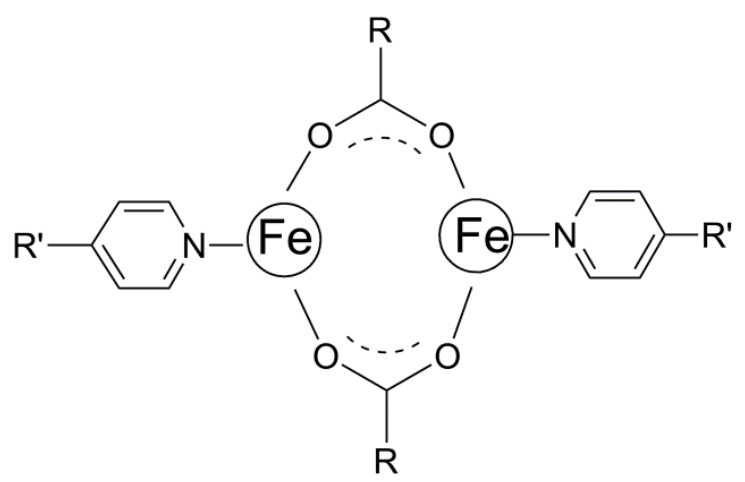

Figure 23. Heteroleptic coordination around iron.

Accordingly, codeposition of an oligophenyl dipyridyl ligands as bis(4-pyridyl)-1,4-benzene (BPB, 1a in Fig. 24) or 4,4'-di(pyridin-4-yl)-1,1'-biphenyl (BPBP, 1b in Fig. 24), together with oligophenyl molecules bearing two carboxylate groups, such as TPA (2a in Fig. 24), BDA ( $2 \mathrm{~b}$ in 
Fig. 24), or [1,1':4',1"-terphenyl]-4,4"-dicarboxylic acid (TDA, 2c in Fig. 24) in combination with $\mathrm{Fe}$ centers ${ }^{327}$ or $\mathrm{Cu}$ centers ${ }^{328}$ on $\mathrm{Cu}(100)$ surfaces yield highly ordered arrays of coordination networks.

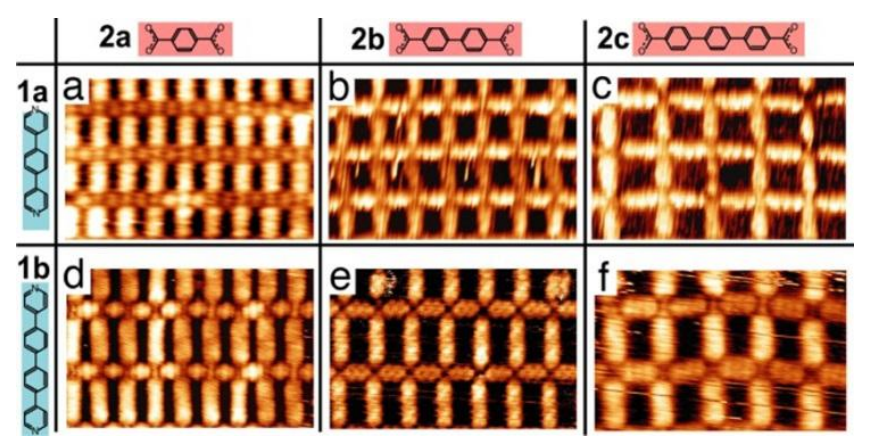

Figure 24. Control of the size and aspect ratio of the supramolecular organization via the lengths of dipyridyl (BPB 1a, BPBP 1b) and dicarboxylic ligands (TPA 2a, BDA 2b, and TDA 2c) in iron-coordinated networks. Reprinted with permission from Ref. ${ }^{327}$. Copyright 2007- National Academy of Sciences, U.S.A.

The internal dimensions of these rectangular compartments are determined directly by the lengths of each ligand, leading to open cavities ranging in area from $1.9 \mathrm{~nm}^{2}$ to $4.2 \mathrm{~nm}^{2}$. Interestingly, the authors have discussed the mechanisms of efficient error correction and cooperativity and have shown how efficient reversibility of the Fe-pyridine versus Fecarboxylate bonds, in addition to the cooperative binding of the components, improved the error correction mechanism.

The same authors reported supramolecular selectivity from a relatively complex mixture of two ligands and two metal atoms. In this experiment, PBP and BDA are coevaporated in a 1:1 ratio onto a $\mathrm{Cu}(100)$ surface at $\mathrm{RT}$. At this temperature, it has been shown ${ }^{323}$ that extended coordination architectures are formed by the complexation of mobile copper adatoms. Then, the temperature is increased to $450 \mathrm{~K}$, and iron atoms are deposited onto the substrate. This annealing process allows the deprotonation of the carboxylic groups and the disassembly of the initial structures, provides mobility, and induces a redistribution of the four building blocks, the 
two molecules, and the $\mathrm{Cu}$ and Fe atoms. The system then is allowed to cool to RT. STM images show a supramolecular segregation into subdomains of $\left[\mathrm{Cu}_{4}(\mathrm{PBP})_{2}\right]_{\mathrm{n}}$ (marked in Fig. 25 by a blue arrow) and $\left[\mathrm{Fe}_{4}(\mathrm{BDA})_{4}\right]_{\mathrm{n}}$ (marked in Fig. 25 by white arrows). ${ }^{329}$ Both structures have been separately reported in previous studies. ${ }^{324,330}$

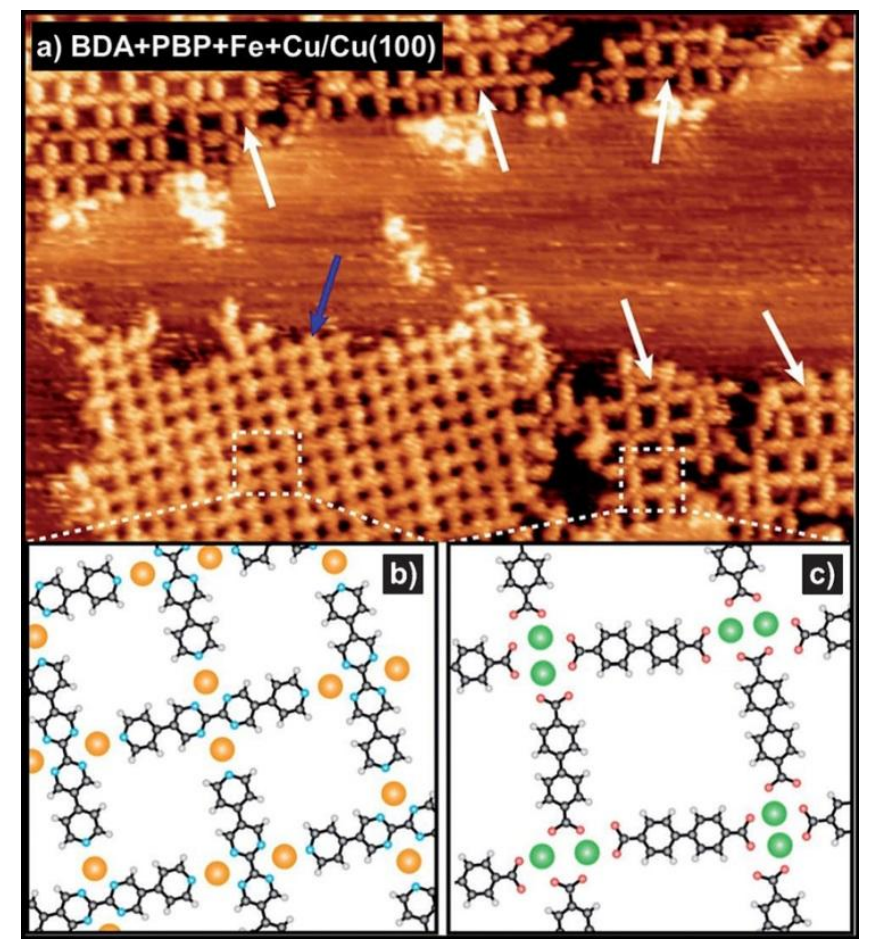

Figure 25. Self-assembly of a mixture $B D A+P B P+C u+F e / C u(100)$ exhibiting phase separation in Cu-PBP (b) and Fe-BDA domains (c). Reprinted with permission from Ref. ${ }^{329}$. Copyright 2015 Wiley.

These segregated phases, in which no heteroleptic coordination is observed, differ from the ladder-type architectures with the similar ligands dicarboxylic and phenyl and diphenyl dipyridyl ligands (BPB and BPBP) and Fe atoms (see above) that were reported by the same group. ${ }^{327}$ This dramatic difference can be understood by comparing the different thermodynamic stabilities of the systems and-in particular - the stability induced by the presence of the pyrimidine chelate. While PBP forms a relatively stable coordination network on its own, the phenyl and biphenyl analogues (BPB and BPBP) show only metastable homoleptic coordination 
on $\mathrm{Cu}(100)$. In brief, the segregation can be explained by the stabilization of $\mathrm{Cu}^{\mathrm{I}}$ by the pyrimidine ligand PBP, while $\mathrm{Fe}^{\mathrm{II}}$ is stabilized by the carboxylate groups of the BDA ligand. Furthermore, the formation of $\mathrm{Fe}^{\mathrm{II}}-\mathrm{PBP}$ leads to a +4 charge per unit cell, which is unlikely to be stabilized by charge screening and therefore induces a destabilization of this hypothetical structure. DFT calculations confirmed that the observed parallel assembly of segregated homoleptic domains is more stable by $1.2 \mathrm{eV}$ than an assembly with reverse metal-ligand pairing or by $2.7 \mathrm{eV}$ for a mixed phase of Fe-PBP-Cu-BDA. This seminal result shows that careful design of the layer components can lead to selective assembly with precise architecture by specific control of the coordination chemistry.

When iron atoms on $\mathrm{Au}(111)$ are complexed by a mixture of molecules comprising the terpyridyl group and molecules with a pyridyl group, the most stable geometry is a linear one, 4',4"'"-(1,4phenylene)bis-2,2'-6',2"-terpyridine-Fe-5,10,15,20-tetra(4-pyridyl)porphyrin

(PTpy-Fe$\mathrm{TPyPH}_{2}$ ), shown in Fig. 26. The network expands using PTpy as linear branches and $\mathrm{TPyPH}_{2}$ as connection corners between these PTpy-Fe branches.

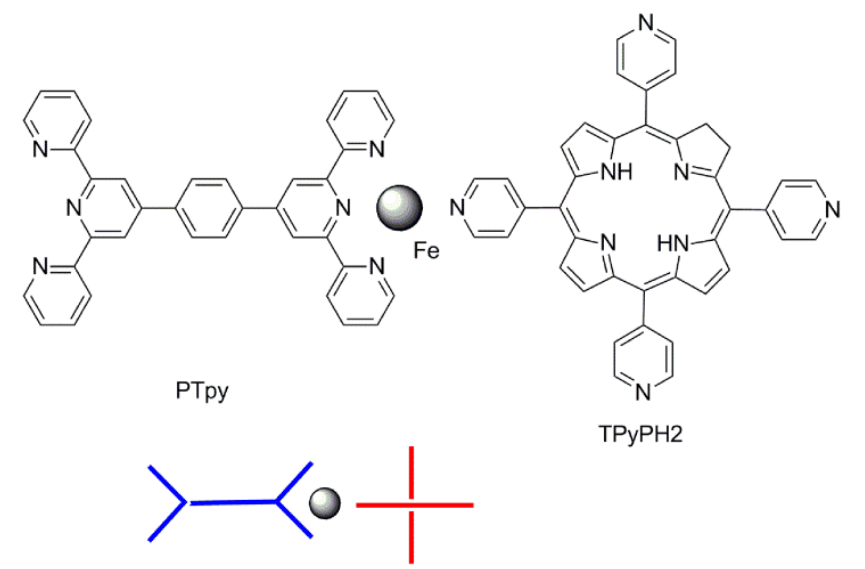

Figure 26. Chemical structure of 4',4"'-(1,4-phenylene)bis-2,2'-6',2"-terpyridine (PTpy) and the free base 5,10,15,20-tetra(4-pyridyl)porphyrin (TPyP $\left.\mathrm{H}_{2}\right)$ showing the preferred coordination geometry around the iron atom. 
For instance, codeposition of iron, PTpy and the free base $\mathrm{TPyPH}_{2}$ (or its zinc complex $\mathrm{ZnTPy}$ ) produces open network structures: either a rhombic structure, a Kagome lattice as shown in Fig. $27,{ }^{331}$ or closed polygons and cages with four components. ${ }^{332}$
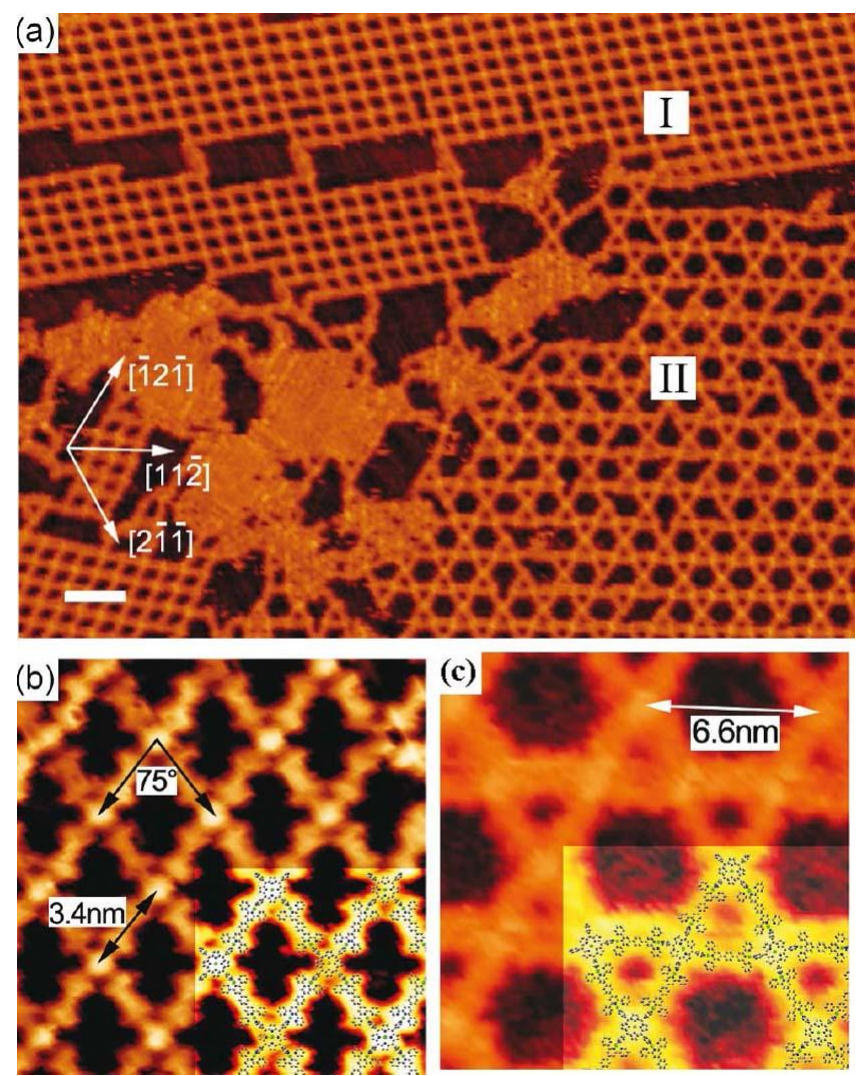

Figure 27. a) STM image of the coexisting rhombic (type I) and Kagome phase (type II); scale bar, $10 \mathrm{~nm} . \mathrm{b}$ ) Model of phase I. c) model of phase II. Reprinted with permission from Ref. ${ }^{331}$. Copyright 2015 ACS.

Rhombic structures are formed by connecting four of the units in Fig. 26, the expected square structure being distorted by interaction with the $\mathrm{Au}(111)$ surface. Similarly, the Kagome structure comprises hexagons formed by six units fused to six triangular units. Here, again, the three-fold symmetry of the gold surface imposes distortions on the flexible coordination geometry around the $\mathrm{Fe}$ atoms. It is possible to selectively obtain pure phases of either type I or type II by adjusting the self-assembly conditions. When the overall molecular dosage is increased with an excess of PTpy, type-I islands cover the surface almost entirely. The fact that 
this phase is favored can be rationalized in terms of entropy differences between the two phases: qualitatively, the Kagome lattice has a lower entropy; thus, its formation is less favored. ${ }^{333}$ The proportion of type-II Kagome phase can be increased using a guest-inclusion approach. A sample of type-I surface was first prepared as indicated above. Then, additional PTpy was deposited on this surface, which was then annealed at $450 \mathrm{~K}$, leading to the appearance of phase II coexisting with phase I. By increasing the dosage of PTpy, more than $95 \%$ of the surface was occupied by phase-II. High-resolution images showed that 1 to 3 guest PTpy molecules appearing as diffusive clouds occupied the hexagonal rings of the Kagome lattice. The proposed mechanism is as follows: PTpy molecules deposited on a phase-I surface are trapped in the network voids of this phase. When the dosage increases, the number of trapped molecules increases, and the repulsion between these guest molecules in this confined environment increases. At the annealing temperature, the small rhombic voids expand under the "pressure" of the guest molecules, forming a phase-II structure with larger voids.

As briefly summarized here, metal-organic network research is a lively area because these structures offer great potential for designing innovative supramolecular networks on surfaces. As described before, most of the studies take advantage of s-, d-, and p-metal (Na, K, $\mathrm{Cs}, \mathrm{Mn}, \mathrm{Fe}, \mathrm{Co}, \mathrm{Ni}, \mathrm{Cu}, \mathrm{Ag}, \mathrm{Au}, \mathrm{Pb} . .$.$) adatoms to bind molecular linkers. Recently, new species$ of adatoms have been used, namely, the lanthanides: cerium, ${ }^{334}$ gadolinium, ${ }^{335,336,337}$ and europium. $^{338}$ This case involves the formation of a bicomponent system composed of quarterphenyl-4,4"-dicarbonitrile and PTpy ligands. The obtained metallosupramolecular structures were based on the 4- and 5-fold coordination sphere of Eu adatoms.

\section{ASSEMBLIES STABILIZED BY NON-DIRECTIONAL INTERACTIONS}


When the intermolecular interactions are weaker and not as directional, the influence of the molecule-substrate interaction grows in importance, and this delicate balance often leads to several stable/metastable supramolecular assembly possibilities.

\subsection{Porphyrins}

An illustration of the substrate influence is the self-assembly of two porphyrin species, the free base tetraphenyl porphyrin $\left(\mathrm{TPPH}_{2}\right)$ and its cobalt complex CoTPP, on various surfaces. ${ }^{339}$ On $\mathrm{Ag}(111)$, an ordered intermixed $\mathrm{TPPH}_{2} / \mathrm{CoTPP}$ layer is found, whereas phase separation occurs on $\mathrm{Cu}(111)$. This observation can be rationalized by the fact that adsorption of the free base is dominated by molecule-substrate interaction, in particular between the iminic nitrogen atoms and the copper surface, whereas the supramolecular arrangement of CoTPP is driven by attractive intermolecular interactions.

An instructive analysis of the thermodynamic aspects of bicomponent self-assembly of two substituted zinc porphyrins has been reported by Wintjes et al. ${ }^{340}$ Both molecules encompass alkane chains substituents, and one contains a cyanophenyl substituent at the meso position. The analyses of several series of experiments at different coverages and different molecular ratios enabled construction of a phase diagram for the bicomponent system and allowed the authors to identify the conditions required to obtain an intermixed phase. A remarkable difference between systems in solution and at the solid-liquid interface is the strong influence of the total amount of molecules on the surface, which can influence the type of bonds occurring, the enthalpy/entropy balance (particularly in the case of flexible molecules), ${ }^{341}$ and diffusive mass transport in the absence of a desorption/readsorption process.

\subsection{Phthalocyanine: penta-tert-butyl-corannulene}


The flexibility of networks maintained by weak H-bonds can be used to allow the insertion of a second component while maintaining a high degree of order in the monolayer. For instance, 2D crystallization of 2,3,9,10,16,17,23,24-octachlorozinc phthalocyanine $\left(\mathrm{ZnPcCl}_{18}\right)$, an electron acceptor, on $\operatorname{Ag}(111)$ transitions from low-density (phase I) to compact packing (phase III) through two phase transitions. ${ }^{342,343}$ This metastability is a result of the balance between attractive intermolecular $\mathrm{C}-\mathrm{Cl}{ }^{\cdots} \mathrm{H}-\mathrm{C}$ bonding, the molecule surface interactions and the substrate-mediated intermolecular repulsions. Deposition of 1,3,5,7,9-penta-tert-butylcorannulene (PTBC), an electron donor, into the two non-compact phases I and II leads to wellorganized supramolecular structures of lines of PTBC inserted in compact lines of $\mathrm{ZnPcCl}_{18}$ (Fig. $28)^{344,345}$ up to two $1: 1$ superstructures.
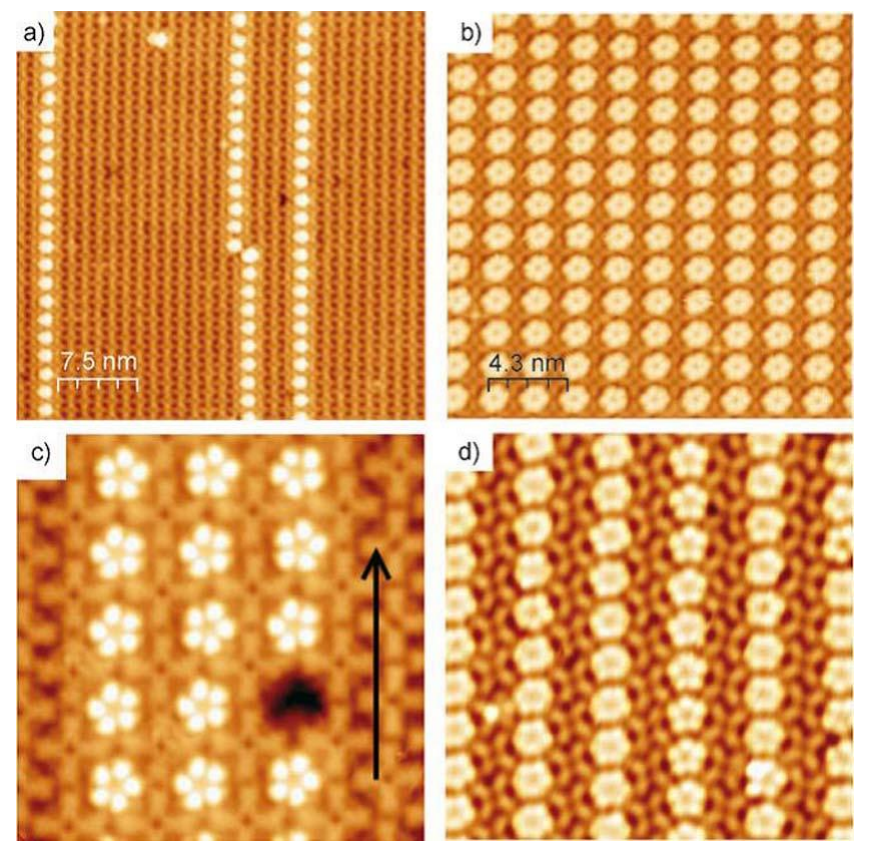

Figure 28. a) STM images of a $\mathrm{ZnPcCl} l_{8}$ network (phase I) after evaporation of PTBC molecules at low coverage; $b$ ) at higher coverage; c) details of the self-assembly showing a vacancy created by STM manipulation of one PTBC. The black arrow shows the propagation direction of the insertion of PTBC. d) Bicomponent network obtained by evaporation of PTBC into phase II. Reprinted with permission from Ref. ${ }^{344}$. Copyright 2015 Wiley. 


\subsection{Phthalocyanine: DIP/pentacene}

Similar behavior has been observed for the codeposition of fluorinated copperphthalocyanine $\left(\mathrm{F}_{16} \mathrm{CuPc}\right)$, an electron acceptor, and DIP, an electron donor, on $\mathrm{Au}(111)$ surfaces. The main difference compared to the previous example is that $\mathrm{F}_{16} \mathrm{CuPc}$ is a perfluorinated phthalocyanine; thus, intermolecular interactions in the single-component layer are weaker. Upon self-assembly of the bicomponent monolayer, the ability to form $\mathrm{C}-\mathrm{F}^{\cdots} \mathrm{H}-\mathrm{C}$ between the phthalocyanine and the DIP greatly increases the intermolecular interactions, which modifies the molecule-substrate distances, weakens the phthalocyanine-Au interaction and reduces the charge transfer to the molecule. This report emphasizes how the control of intermolecular interaction, by choosing the correct chemical functions, could be a way to modulate the molecule-substrate electronic coupling and therefore to tune charge carrier injection in optoelectronic devices. Comparisons with binary networks of DIP with nonfluorinated CuPc have also been reported ${ }^{346,347}$ that allow the realization of molecular templates with tunable symmetry and periodicity.

When pentacene is used instead of DIP, ${ }^{348}$ coadsorption with $\mathrm{F}_{16} \mathrm{CuPc}$ on $\mathrm{Cu}(100)$, in a ratio of $2: 1$, results in a $2 \mathrm{D}$ binary layers of a crystalline nanoporous network with two enantiomorphic chiral domains. Here, again, the enhanced intermolecular interaction by the formation of $\mathrm{C}-\mathrm{F}^{\cdots} \mathrm{H}^{-}$ $\mathrm{C}$ bonds weakens the molecule-substrate electronic coupling. A study of this bicomponent system on $\mathrm{Au}(111)^{349}$ has focused on the alloying, solid solutions, phase separation and segregation as influenced by the molecular ratios. The delicate balance between intermolecular and molecule-substrate interactions and its effect on molecular self-assembly has been illustrated by studying two similar blends of $\mathrm{F}_{16} \mathrm{CuPc}$ :Pen and $\mathrm{CuPc}$ : perfluoropentacene $\left(\mathrm{F}_{14} \mathrm{Pen}\right)$ on $\mathrm{Au}$ surfaces (Fig. 29). ${ }^{350,351}$ 


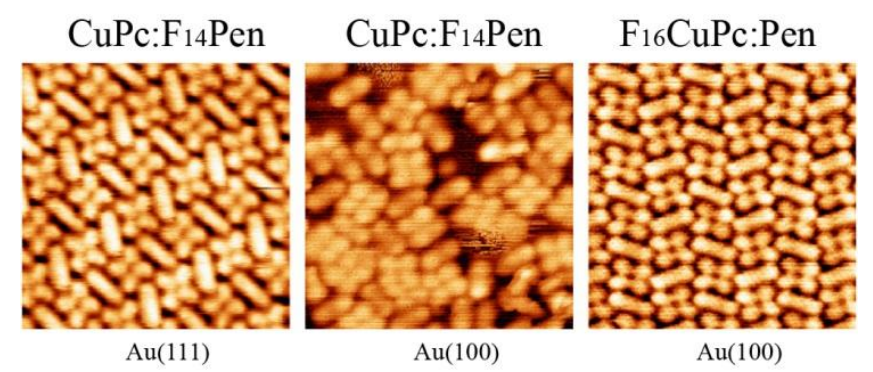

Figure 29. Asymmetric response towards molecular fluorination in binary fluorinated CuPc:Pen assemblies as influenced by substrate faces. Reprinted with permission from Ref. ${ }^{350}$. Copyright 2015 ACS.

Deposition of the blend $\mathrm{CuPc} / \mathrm{F}_{14} \mathrm{Pen}$, a bicomponent system with weak intermolecular interactions, on $\mathrm{Au}(100)$, a surface with high molecule-surface potential corrugation, leads to a disordered monolayer (Fig. 29, center). On Au(111), the corrugation of the molecule-substrate potential is smaller, and the molecule-molecule interactions predominate to yield an ordered 2D crystal (Fig. 29, left). In contrast, stronger intermolecular interactions in $\mathrm{F}_{16} \mathrm{CuPc} / \mathrm{Pen}$ blends produce a 2D crystalline network, even on $\mathrm{Au}(100)$ (Fig. 29, right).

A complementary study of coadsorption of $\mathrm{F}_{16} \mathrm{CuPc}$ on $\mathrm{HOPG}$ with DIP, Pen and 6P demonstrated that control of the molecular ratio can lead to a variety of phases. ${ }^{352}$ In these phases, the structural stability is maintained essentially by intermolecular H-bonds, and the molecule/graphite interface is dominated by $\pi-\pi$ interactions. The smooth potential energy surface of HOPG does not induce any interlocking of the molecules with the substrate lattice. The self-assembly of $\mathrm{F}_{16} \mathrm{CuPc}$ and $\mathrm{CuPc}$ has also been investigated on $\mathrm{HOPG}^{353}$ At low $\mathrm{CuPc}$ coverage, the $\mathrm{CuPc}$ is randomly embedded in a $\mathrm{F}_{16} \mathrm{CuPc}$ matrix; as the $\mathrm{CuPc}$ coverage increases to $50 \%$, the bicomponent system is transformed to form a highly stable $1: 1$ chessboard-like pattern by intermolecular H-bonding. The coadsorption of 1,3,5-tris $[(E)-2-(3,5-$ 
didecyloxyhenyl)-ethenyl]-benzene with coronene or/and $\mathrm{HBC}$ on HOPG produces a fine 2D molecular sieve. ${ }^{354}$

$\mathrm{Cu}$-phthalocyanine $(\mathrm{CuPc})$ has also been codeposited with PTCDA. ${ }^{355}$ Sublimation of the latter on $\mathrm{Cu}(111)$ followed by PTCDA offered a symmetrical arrangement organized with one $\mathrm{Cu}-\mathrm{Pc}$ and two PTCDA molecules in close vicinity. The exact positions of the molecules on the $\mathrm{Cu}(111)$ lattice could not be determined by STM, but in this specific case the symmetry is induced by mutual interaction between the molecules and substrate; thus, the CuPc molecules are expected to be aligned along the corresponding row of $\mathrm{Cu}$ atoms. PTCDA is expected to bind to the substrate preferentially by the three oxygen atoms at each side of the molecule. Based on this scheme, the authors concluded that the PTCDA molecules form a zigzag chain as part the highly stable hexagonal structure observed on a monolayer of pure PTCDA. For the same reason, mixed layers of PTCDA and CuPc are not found if the PTCDA is deposited first: the binding strength of this homomolecular array is too high to be overcome and leads to a mixed system.

To complete the discussion on this family of molecules, it is worth mentioning that binary layers combining $\mathrm{F}_{16} \mathrm{CoPc}$ and NiTPP or $\mathrm{CoPc}$ on $\mathrm{Au}(111)$ exhibit similar well-ordered structures, whereas a mixture of NiTPP and CoPc forms densely packed and well-structured architectures but with compositional disorder. ${ }^{356,357}$ The geometric and electronic similarities between the two species lead to solid-solution 2D crystallization similar to what has been observed in the case of binary layers of $\mathrm{CuPc}$ and $\mathrm{CoPc}$ on $\mathrm{Au}(111){ }^{358}$

\subsection{Sub-phthalocyanine}


The balance between electrostatic and vdW interactions has been described in the case of the coadsorption of chloro[sub-phthalocyaninato]boron(III) (SubPc-Cl, Fig. 309) and $\mathrm{C}_{60}$ on $\operatorname{Ag}(111){ }^{359}$

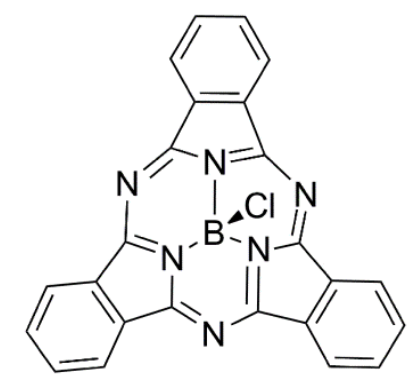

Figure 30. Chemical structure of chloro[sub-phthalocyaninato]boron(III) (SubPc-Cl).

The adsorption of SubPc-Cl on $\mathrm{Ag}(111)$ has been reported to be dominated by the $\mathrm{Ag}-\mathrm{Cl}$ interaction, with the $\mathrm{B}-\mathrm{Cl}$ bond pointing towards the surface, ${ }^{360}$ whereas the adsorption on $\mathrm{Au}(111)$ is via the isoindol system, with the $\mathrm{B}-\mathrm{Cl}$ bond pointing up; ${ }^{361}$ on $\mathrm{Cu}(100)$, the molecule can flip between both configurations. ${ }^{362}$ On $\mathrm{Ag}(111)$, at sub-monolayer coverage, SubPc-Cl forms non-compact $2 \mathrm{D}$ periodic islands in equilibrium with a $2 \mathrm{D}$ gas of diffusing molecules. The permanent dipole moment of the molecule and its image charge in the substrate create an important repulsive lateral interaction between the molecules, resulting in the distances between these molecules $(1.79 \mathrm{~nm})$ being too far for $\mathrm{vdW}$ contact. When $\mathrm{C}_{60}$ and $\mathrm{SubPc}-\mathrm{Cl}$ are codeposited on $\mathrm{Ag}(111)$, islands of mixed phases appear as stripes with a fixed $\mathrm{SubPc}-\mathrm{Cl}: \mathrm{C}_{60}$ ratio of $2: 3$, as shown in Fig. $31 .^{359}$ 


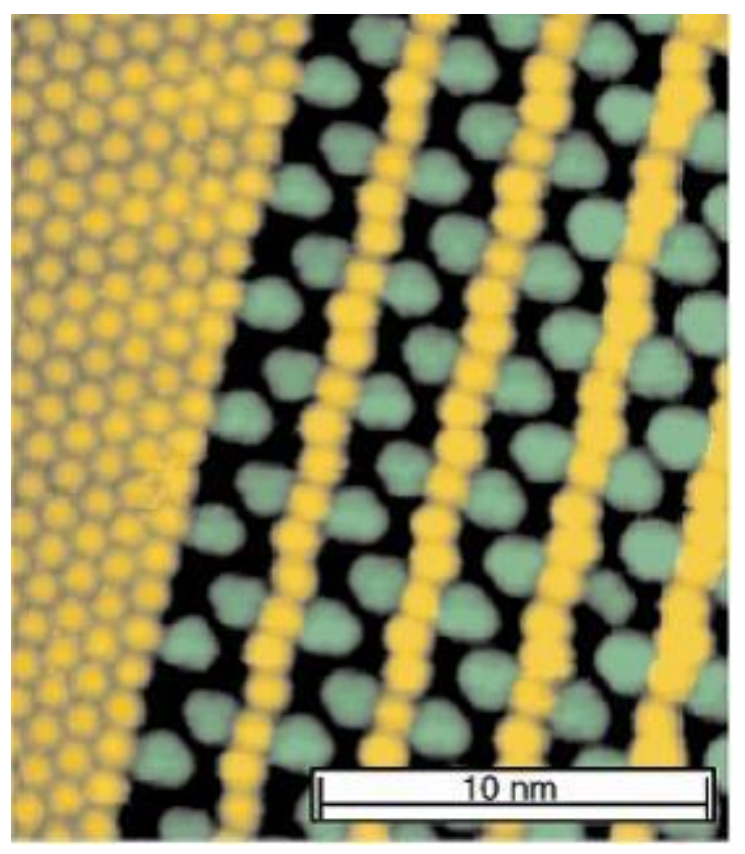

Figure 31. Bicomponent "stripe" phase of $C_{60}$ (yellow dots) and SubPc-Cl (green dots) with a pure $C_{60}$ island (left). Reprinted with permission from Ref. ${ }^{359}$. Copyright 2015 Wiley.

Higher SubPc-Cl coverage leads to a new hexagonal ("star") phase coexisting with the stripe phase, with a molecular ratio of 1:1. STM images also show a $2 \mathrm{D}$ gas of diffusing molecules between these two patterns. Interestingly, the packing density of SubPc-Cl in the "stripe" and "star" phases is 7\% and 20\%, respectively, higher than in the honeycomb phase of pure SubPc-Cl. This observation suggests a higher binding energy arising from increased attraction between antiparallel dipole moments generated by the adsorption of the two species, intermolecular vdW and anisotropic Coulomb interactions.

\subsection{TTF-TCNQ}

The tetrathiafulvalene-tetracyanoquinodimethane (TTF-TCNQ) molecular solid phase is a wellknown organic metal ${ }^{363}$ with a $1 \mathrm{D}$ band structure due to the partial electron transfer between the donor TTF and the acceptor TCNQ; thus, it is not surprising that several groups have explored 
the possibility of tuning organic-metal hybrid bands using this bicomponent system. On $\mathrm{Au}(111),{ }^{364}$ both TTF and TCNQ lie parallel to the surface in a mixed phase as shown in Fig. 32. The molecular layer is bonded to the gold surface through TTF via two S-Au bonds per molecule as in homomolecular layers. ${ }^{365}$ In contrast, TCNQ is physisorbed and interacts weakly with the substrate.

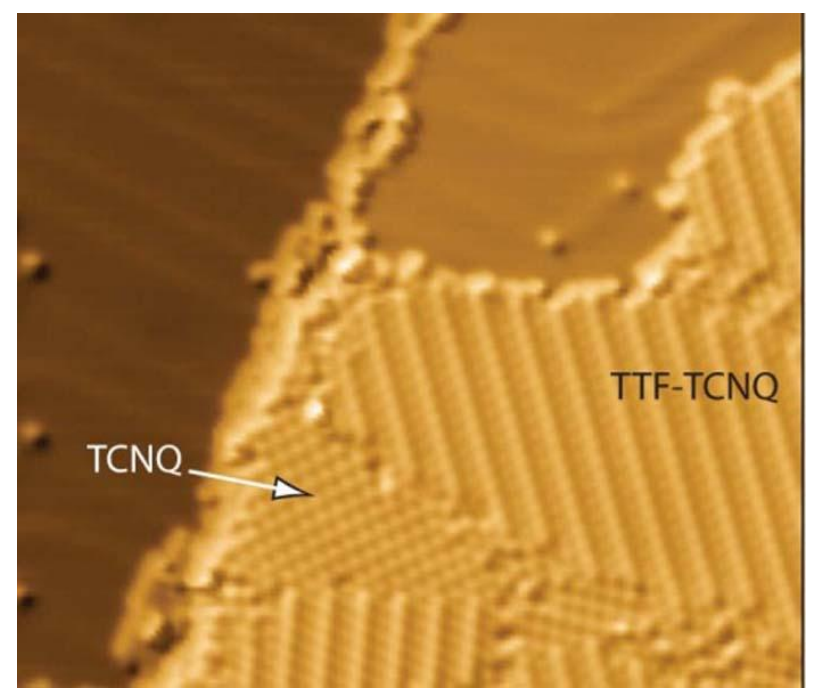

Figure 32. TTF-TCNQ domains on Au(111). Excess TCNQ molecules appear segregated in homomolecular islands. Reprinted with permission from Ref. ${ }^{364}$. Copyright 2015 APS.

In the homomolecular layers, the charge distribution upon adsorption of the donor TTF generates intermolecular long-range Coulomb repulsion, leading to low-density packing and long intermolecular distances, as observed for SubPc-Cl/Ag(111) (see above). In the mixed phase, although the interaction of the TTF-TCNQ layer on Au(111) is conducted essentially by the TTF molecule, the presence of TCNQ allows tuning of the strength of the donor-metal interaction and of the spacing between TTF. This configuration with chemisorption of one type of molecule and physisorption of the other species in the same monolayer is unusual in supramolecular bicomponent systems and could provide a useful way to control the interface properties. ${ }^{366}$ 


\section{7. $\mathrm{C}_{60}$ and pentacene}

Guest et $a l .{ }^{367}$ have described the structures formed when $\mathrm{C}_{60}$ and Pen are sequentially deposited on a $\mathrm{Cu}(111)$ surface. When $\mathrm{C}_{60}$, an electron acceptor, is sublimated on the highly ordered and dense herringbone phase of pentacene, an electron donor, a mostly disordered adlayer of $\mathrm{C}_{60}$ is observed with little rearrangement of the dense Pen layer underneath. In contrast, when $\mathrm{C}_{60}$ is deposited on a lower density random tiling Pen phase, i.e., when the Pen phase has more space to reorganize, rearrangement proceeds through the formation of domains based on supramolecular chiral pinwheels as shown in Fig. 33.
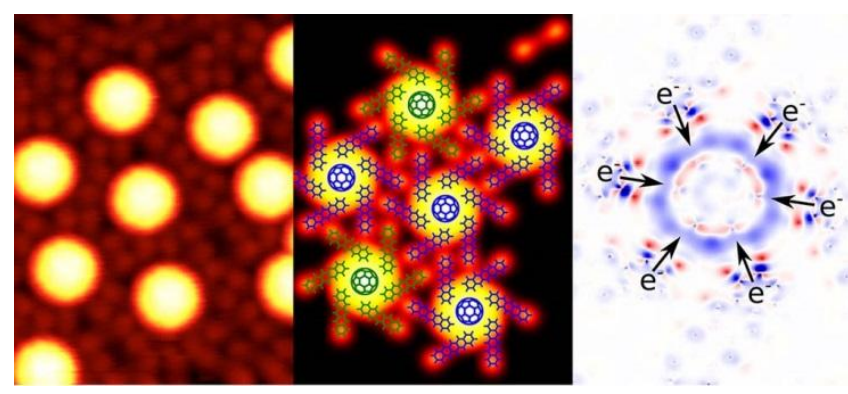

Figure 33. Left: STM image of $C_{60} / P e n / C u(111)$. Center: Model of the heterostructure based on a tiling of both chiralities of pinwheels of Pen molecules. Right: Computed charge density difference for the pinwheel structure (Cu(111) substrate not included in the calculation). In blue, charge depletion. Reprinted with permission from Ref. 367. Copyright 2015 ACS.

VdW-corrected DFT calculations confirm that charge transfer occurs between Pen and $\mathrm{C}_{60}$ and that this effect is enhanced when the molecules are assembled in the pinwheel geometry on the $\mathrm{Cu}(111)$ surface to provide stability to the bicomponent system.

Similarly, well-ordered $\mathrm{C}_{60}$-Pen layers have been found on $\operatorname{Ag}(111)$ with various structures. $^{368}$ The 2D crystallization was tuned by temperature; here, the weak Pen- $\mathrm{C}_{60}$ interaction is dominated by the molecule-silver surface interaction. 


\section{OUTLOOK}

In this last part, we will introduce several research directions along these lines, in particular in fields for which well-controlled tuning of bicomponent interfaces is an important challenge, such as non-metallic surfaces and organic thin films.

\subsection{Towards predicting bicomponent self-assembly: simulations}

Developing the ability to use computational tools to predict the outcome of bicomponent selfassembly is an essential objective that is expected to aid in the design of suitable components. The result of a self-assembly process can be anticipated by examining the balance between the energy inside the layer (interactions between the molecules of the coating) and the molecule/substrate interaction. If we define $E_{\mathrm{MS}}$ as the molecule-surface energy, $E_{\mathrm{MM}}$ the intermolecular energy, $E_{\mathrm{T}}$ the energy associated with the kinetic effect due to a thermostat, and $E_{\text {diff }}$ the diffusion energy of a molecule on the surface between neighboring adsorption sites, these energies can be ordered as follows:

$$
E_{\mathrm{MS}}>E_{\mathrm{MM}} \geq E_{\mathrm{T}}>E_{\text {diff. }}
$$

This inequality can be explicated as follows: if $E_{\mathrm{MS}}$ is not the largest energy, the system is in equilibrium between adsorbed molecules and molecules in the gas phase, so that the condition for self-assembly is not fulfilled at all. If $E_{\mathrm{MM}}>E_{\mathrm{MS}}$, then the molecules on the surface tend to self-aggregate, as it is the usual case for large $\pi$-conjugated molecules forming strong aggregates by $\pi$ stacking. Then, the energy provided by the thermal bath has to be lower than these two previous ones to ensure that the molecules do not desorb. Finally, if $E_{\mathrm{diff}}>E_{\mathrm{MM}}$, the molecules do 
not diffuse and are pinned on single specific adsorption sites. For a more complete discussion, refer to the paper of Hooks et al. ${ }^{369}$

However, this approach is oversimplified and applicable in broad terms only because it does not incorporate the complexity of the potential energy surface corresponding to the manybody system (molecules and the surface) and because it provides no information about kinetic processes occurring during self-assembly. A recent tutorial review by Palma et al. thoroughly addresses these issues. ${ }^{370}$ In particular, the authors emphasize that "self-assembly into large extended architectures (2D crystalline motifs) is possible only through strong enthalpy/entropy compensation", this compensation being introduced by the substrate in our case. It is useful to describe the thermodynamic equilibrium of these systems to identify the parameters (temperature, molecular deposition rate...) that control their ordering, their cohesion and stability (reversibility, phase transitions...). However, the domain sizes and the characteristic times of ordering are generally inaccessible by conventional simulation methods. Phase transitions and superstructures symmetry observed experimentally usually involve several hundred molecules, even several thousands. A huge number of parameters are thus required for atomistic simulations. Moreover, molecular ordering on a surface may take hours to reach large domains, due to a slow release of constraints originating from the incommensurability between the molecular scaffold and the registry of the surface. This process occurs, for instance, with $\mathrm{ZnPcC}_{18}$ molecules on $\mathrm{Ag}(111),{ }^{344,342}$ for which a maturation process at $\mathrm{RT}$ was observed with a structural evolution through three extended phases over almost fifty hours. Finally, the structure of the surface itself can be an obstacle due to complicated reconstructions (semiconductor surfaces), a long-range structure (the well-known $22 \times \sqrt{3}$ structure of the $\mathrm{Au}$ surface) or the presence of defects. Consequently, there is a special need for efficient numerical methods to 
simulate the self-assembly of mono- or bicomponent systems. The most straightforward and broad techniques are the molecular dynamics and Monte Carlo (MC) simulations with classical force fields. Simulations of heteroepitaxy have generated notable results by considering the growth of organic molecules (PTCDA, titanylphthalocyanine, or pentacene) on inorganic surfaces [HOPG, $\mathrm{Au}(111)$, or $\mathrm{BN}]$ or on organic layers (HBC on $\mathrm{HOPG}) .{ }^{371,372,373,374}$ An all-atom molecular dynamics scheme was developed by Samorì and coworkers and was implemented in the program CHARMM. ${ }^{375,376}$ The use of an implicit graphene substrate and periodic boundary conditions enabled the simulation of bicomponent self-assembly ${ }^{377}$ with a stoichiometric mixture of 80 melamine and bis(N1-hexyl-uracil) molecules and provided a description of the selftemplating of bidimensional Kagome supramolecular networks with a fully planar tetracarboxylic acid derivative. ${ }^{378}$ By using standard MC procedures for diffusive particles on a substrate, ${ }^{379}$ Taylor and Beton have described the growth of 1D structures on a model hexagonal surface by mimicking molecules with a simple uniaxially anisotropic intermolecular potential. $^{76,77,78,79}$

Similarly, on-lattice kinetic MC (KMC) simulations have attracted interest in the context of self-assembly modeling with one and two components. ${ }^{294,380,381,48,102,382,322,383,384}$ Additionally, a notable study using KMC simulations showed that the conditions for realizing stable molecular networks of a bicomponent system are based on the ratio between the directional and isotropic coupling energies for the molecules. ${ }^{385}$ A structural stability diagram was obtained that sets the conditions for disordered, compact and network domains according to the temperature and the strength of the H-bonding and vdW energies. This framework was validated using the melaminePTCDI molecular system on a model hexagonal lattice, for which the complementary H-bonding favors the formation of a 2D open honeycomb network. The agreement with experimental results 
(PTCDI-melamine on $\mathrm{Ag} / \mathrm{Si}(111)-\sqrt{ } 3 \times \sqrt{ } 3 R 30^{\circ}$ ) is remarkably good. Although outside the scope of the present review, it is worth mentioning a preliminary model of bicomponent network formation at the solid/liquid interface. ${ }^{386}$ With the help of DFT calculations, the conditions of mixed and de-mixed assembly, of coexistence of multiple segregated phases, or of polymorphism according to the relative amount of melamine and four-linear-linker bonding via triple H-bonds have been described. The authors indicated that "polymorphism is an intrinsic feature of molecular design". Such a conclusion is expected to be illuminating for UHV studies. Moreover, the description of the first stages of a self-assembled layer formation of $\mathrm{C}_{60}$ on an SAM also at the solid/liquid interface ${ }^{387}$ has been approached both experimentally and by computation.

As already mentioned, a computational limitation exists due to the number of adsorbed molecules that can be considered in a total system. An approach to overcome this limitation is to consider an alternative method with a coarse-grained model (briefly, the coarse-graining consists of neglecting an atomistic description in favor of single and larger beads representing chemical functional groups, as described for instance in the MARTINI method ${ }^{388}$ ), which was applied for organic molecules on graphite ${ }^{389}$ or on semiconductor surfaces ${ }^{390}$ with relative success in terms of the system size that could be accommodated. We also note a promising methodology combining kinetic and thermodynamic properties that is able to simulate the entire molecular self-assembling process and is termed self-assembly of nano-objects (SANO). ${ }^{391}$ Based on a 2D grand canonical MC approach and fast interpolation of an acute and large grid of pre-calculated potential energies (molecule-molecule and molecule-substrate interactions), the SANO code calculates various self-assembled arrangements starting from the bare surface and by varying the deposition temperature and then the quenching temperature. The authors explain that the 
calculation of potential energies is achieved either with a suitable force field or with a quantum mechanical method. Despite the fact that the internal degrees of freedom of the adsorbates and the substrate are not taken into account, this method has produced valuable results for selfassembled systems such as octachloro-zinc-phthalocyanines $\left(\mathrm{ZnPcCl}_{8}\right)$ on $\mathrm{Ag}(111)$, copperphthalocyanines-fluorinated $\left(\mathrm{CuPcF}_{16}\right)$ on $\mathrm{Au}(111)$, and penta-tert-butylcorannulene (PTBC) on $\operatorname{Ag}(111)$. Recently, the self-assembly of DIP at RT on different reconstructions of the $\mathrm{Au}(111)$ surface was reported, emphasizing the complex role of the driving force (either cooperative or competitive) in producing long-range ordered domains and surface-induced chirality consistent with experimental STM results. ${ }^{392}$ Even though the actual studies are devoted to the selfassembly of a single molecular species, the SANO numerical tool can be extended formally to simulate the growth of 2D supramolecular networks with bicomponent systems.

\subsection{Towards higher complexity: multicomponent systems}

Proceeding to higher complexity while maintaining control in this field is attractive since it would allow precise tuning of supramolecular network properties and molecule-substrate interaction. Additionally, one of the possibilities involved in this objective is to increase the number of molecular components. However, as can be easily inferred from the above discussion, the number of parameters to master and the number of possible meta-stable geometries increase rapidly with the number of components, such that it is difficult to predict ab initio a supramolecular arrangement or even to rationalize the observations; thus, it is not surprising that such examples are scarce.

Tricomponent systems have been extensively studied on HOPG in liquid, but only a few examples can be cited for metallic surfaces under UHV. In 2008, Bonifazi et al. reported the first 
example of simultaneous three-component assembly on $\operatorname{Ag}(111)$ mediated by triple H-bonding interactions at low temperature. ${ }^{393}$ They observed supramolecular linear assembly by three Hbonds between 2,6-di(acylamino)pyridyl (DAD) and uracil (ADA). The third component, an anthracyl unit connected to one uracil group, acts as a molecular stopper with 2,6di(acylamino)pyridyl at the end of the "bicomponent chain". Actually, the chain length is not controlled, and the third component is not strictly involved in the network. In 2010, Gardener and co-workers reported a three-component network (Fig. 34) by codeposition of uracil, PTCDI and melamine on $\mathrm{Au}(111)$ at $\mathrm{RT},{ }^{394}$ opening novel perspectives in this field but also highlighting the complexity of such systems.

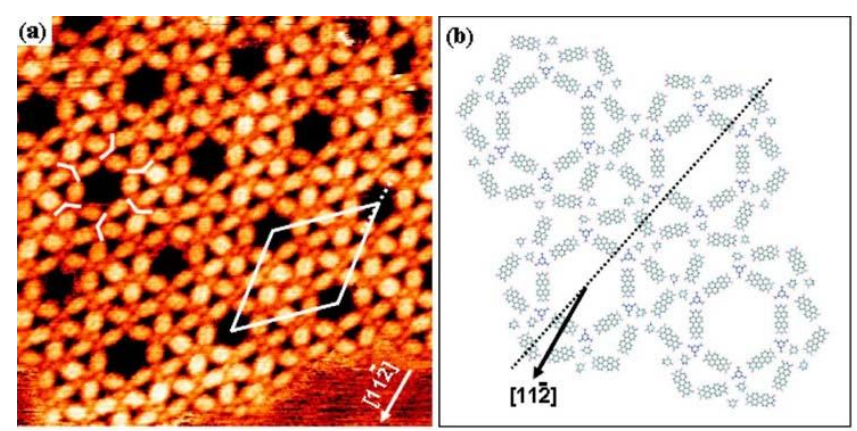

Figure 34. (a) Chevrons based on a combination of M, uracil and PTCDI on Au(111). The unit cell is depicted by a white parallelogram, and the dashed line is a symmetry axis of a hexagonal pore. White chevrons overlap the cluster of three molecules extending from the hexagonal apex. (b) An image representing a possible arrangement of the structure from the same region with the same chirality and pores of several dimensions. Reprinted with permission from Ref. ${ }^{394}$. Copyright 2015 ACS.

In this three-component network, the assembly consists of large hexagonal pores, each of which is surrounded by six smaller triangular voids. This organization is different from what was observed for the bicomponent system PTCDI-melamine (see 3.1.2.2 above). Thus, even though uracil and melamine could not be distinguished by STM (because of their similar size), this change in network organization from adding a new component allowed the authors to deduce the 
presence of a third component. This network is unique in that it contains two cavities with different sizes and could be used to host two different species.

\subsection{Non-metallic surfaces}

To date, a large majority of self-assembled systems have been observed on conducting substrates. However, when considering future applications in electronics or optics, there is a substantial interest in extending this research field to insulating and semiconducting surfaces.

On insulating surfaces, such as those of dielectric crystals, the molecule-substrate interaction is generally weak, leading to a high mobility, clustering at step-edges ${ }^{395}$ and the formation of bulk crystals, ${ }^{396}$ the intermolecular interaction being higher than the moleculesurface interaction. One article reported the co-adsorption of two molecular species, PTCDI and $\mathrm{C}_{60}$, on an insulating surface, namely, $\mathrm{CaF}_{2}(111) .{ }^{397}$ PTCDI layers are known to grow on $\mathrm{NaCl}(001)^{276}$ in a bimodal mode. NC-AFM images taken within a few hours of deposition reveal square-shaped monolayer islands and multiple layers of needle-shaped islands. At RT, the metastable monolayer islands shrink and disappear within days, while the stable multilayer islands grow larger, indicating a dewetting of the surface. $\mathrm{C}_{60}$ monolayers islands have also been

observed at step-edges of $\operatorname{KBr}(001)^{398}$ during the initial steps of nucleation of multilayer islands. Successive deposition of PTCDI followed by $\mathrm{C}_{60}$ led to PTCDI islands covered by $\mathrm{C}_{60}$, whereas deposition of $\mathrm{C}_{60}$ followed by PTCDI produced essentially pure islands of PTCDI. Nevertheless, simultaneous deposition of both molecules resulted in a new phase, which has been tentatively assigned to islands of intermixed phases. However, the lack of molecular resolution did not allow a precise description of packing in this bicomponent phase. 
This field is currently burgeoning, and there is a need to develop bicomponent supramolecular assembly on insulating surfaces by simultaneously maximizing intermolecular (e.g., by complementary H-bonding) and molecule-substrate interactions (e.g., using electrostatic forces in the form of dipoles ${ }^{399,400}$ and local charges ${ }^{401}$ ).

Several examples using semiconducting surfaces have been reported in the literature. A pioneering work concerns non-covalent self-assembly with two components on a silicon surface. ${ }^{402}$ This study employed 1,3,5-tri(4'-bromophenyl)benzene and 1,3,5-tri(4"-bromo-4,4'biphenyl)benzene deposited on a $\operatorname{Si}(111)-\mathrm{B} \sqrt{ } 3 \times \sqrt{ } 3 R 30^{\circ}$ reconstructed surface. This surface exhibits a behavior dictated by depopulated dangling bonds due to boron atoms just underneath the top silicon layer, providing relatively weak molecule-surface interactions; as a result, adsorbates can diffuse easily and form a supramolecular network with the two aforementioned molecules. Interestingly, the network comprises nanopores with a size well suited for a $\mathrm{C}_{60}$ molecule.

\subsection{Nanopores}

Another recent development has been the exploration of nanoporous networks prepared by supramolecular monocomponent, bicomponent and metal-organic coordination assembly (and more recently by bicomponent covalent coupling). ${ }^{403,404,405,282,406}$ Such networks, featuring periodic empty domains with controllable shapes, sizes and chemical functionalities, are of particular interest for a broad range of applications. For instance, chiral nanopores could be used for enantioselective adsorption of guest molecules. Nanopores could also be reaction vessels for confined syntheses. This active field has been reviewed several times ${ }^{45,46,310,407,408}$ An interesting development has been the functionalization of the inner parts of the pores ${ }^{409,286}$ via the addition of simple functional groups to the molecular building blocks. The selection of side chains allows 
control over the trapping properties of the cavities. This result represents important progress towards chemical selectivity of the nanopores.

\subsection{Organic thin films}

Over the past decade, organic thin films have attracted attention due to their potential as crucial components in low-cost, flexible plastic electronic devices, such as OLEDs, OFETs, POVs and organic spintronics. Much research effort has been devoted to the control and understanding of multilayers systems in which a second layer of molecules is deposited on an organic "buffer layer", which is itself self-assembled on an inorganic substrate. These layers act as structural templates for the second layer and modify the charge carrier (or spin) transport and injection properties of the device. So far, most of these bicomponent systems involve fullerenes, porphyrins and phthalocyanines, and PTCDA for their known electron donor or acceptor properties. ${ }^{410}$ In these systems, the deposition of a second layer of molecules on the first selfassembled film does not deeply perturb this first layer, which can be considered a structural template substrate. Elegant examples involve $\mathrm{C}_{60}$ on cyanoporphyrins, ${ }^{46,411,412} \mathrm{C}_{60}$ on phenoxyphthalocyanine, ${ }^{413} \mathrm{C}_{60}$ on a-sexithiophen, ${ }^{414,415,416,417}$ the reaction of $\mathrm{C}_{60}$ with a porphyrin monolayer, ${ }^{418}$ the assembly of $\mathrm{C}_{60}$ on cerium porphyrin double-decker $\mathrm{Ce}(\mathrm{TPP})_{2},{ }^{419} \mathrm{C}_{60}$ on hexaazatriphenylene-hexanitrile, ${ }^{420} \mathrm{C}_{60}$ on acridine-9-carboxylic acid (ACA), ${ }^{421} \mathrm{C}_{70}$ on titanyl phthalocyanine, ${ }^{422} \mathrm{CuPc}$ on $\mathrm{C}_{60}$ precovered surfaces, ${ }^{423,424} \mathrm{PTCDA}$ on $\mathrm{HBC}^{425}$ or $\mathrm{CuPc},{ }^{426}$ and $\mathrm{F}_{16} \mathrm{CuPc}$ atop binary DIP: $\mathrm{F}_{16} \mathrm{CuPc}$ or $6 \mathrm{P}: \mathrm{F}_{16} \mathrm{CuPc}^{427}$ Recently, Stadtmüller et al. $^{428,429}$ showed that upon deposition of CuPc on a monolayer of PTCDA/Ag(111), the enhanced charge transfer from the substrate to the bilayer did not involve CuPc electronic states (the second layer) but only the PTCDA layer with a strengthening (shortening) of the PTCDA-Ag(111). ${ }^{241}$ The authors suggested that the second layer of $\mathrm{CuPc}$ molecules can be treated as a polarizable medium, 
enabling an additional screening mechanism assisting the screening by the surface image charges. So far, this exploration has been essentially limited to commercially available molecules, but it is likely that this field will reveal its full potential with tuned and customized molecules. ${ }^{412}$

\section{Conclusion}

In this contribution, we have reviewed a selection of the most important results to date in bicomponent supramolecular self-assembly at the vacuum-solid interface. In this field, the selection of molecular systems and surfaces has relied on simplified rules of thumb, often leading to unexpected geometries. These observations have generated extensive understanding of the parameters driving the self-assembly processes and have helped to illustrate the relative roles of intermolecular and molecule-substrate interactions and bonds. To go beyond current empiricism, it is now important to move towards a more predictive approach aimed at designing molecular interfaces with targeted properties. It is only under this condition that on-surface selfassembly will reveal its full innovation potential.

\section{AUTHOR INFORMATION}

\section{Corresponding Author}

*E-mail: andre.gourdon@cemes.fr Phone: +33562257859

\section{Notes}

The authors declare no competing financial interest.

\section{Biographies}

Xavier Bouju (born 1967) obtained his PhD at the University of Franche-Comté (Besançon), under the supervision of Christian Girard (1993). He devoted one year to national military 
obligations (1994) and then moved to the University of Namur for a post-doctoral appointment with Prof. Vigneron (1995). He then returned to France, where he was appointed as a CNRS research scientist in the Molecular Physics Laboratory (Besançon) between 1996 and 2002. Then, he moved to Toulouse in the CNRS-CEMES laboratory and was promoted to senior CNRS research director (2013). His main research interests are focused on numerical simulations of adsorbed molecules in the context of self-assembly on surfaces, image calculations with localprobe-based methods (STM, AFM), and simulations of adsorbed and functionalized molecular machines.

Cristina Mattioli was born in Reggio Emilia, Italy, in 1985. She received her M.Sc. in Chemistry in 2010 from the "Università degli Studi di Parma", Italy. Her focus was the synthesis and functionalization of calix[4]arene-stabilized gold nanoparticles and the study of their selfassembling properties with pyridinium salts in apolar media. After her M.Sc., she worked under the supervision of Professor A. Arduini on the synthesis of dialkyl-viologen salts to be employed as axles in calix[6]arene-based rotaxanic structures. In 2011, she joined the group of Dr A. Gourdon in the laboratory "Center d'Elaboration de Matériaux et d'Etudes Structurales" of Toulouse, France; she submitted her PhD thesis on the synthesis of molecular systems capable of self-assembly on epitaxial graphene surfaces in UHV in 2014.

Grégory Franc obtained his MSc degrees at Ecole des Mines d'Albi and Institut National Polytechnique of Toulouse in 2004. He subsequently joined the group of Dr Majoral and Dr Caminade (Laboratoire de Chimie de Coordination in Toulouse) for his graduate studies and obtained his PhD from Université Paul Sabatier in 2007. He then received training as a 
postdoctoral fellow under the supervision of Prof. A. Kakkar (McGill University, Montreal) and Dr A. Jutand (Ecole Normale Supérieure, Paris) and finally Dr A. Gourdon (CEMES, Toulouse). Since 2012, he has been working as a consultant specializing in IT service management.

Adeline Pujol studied at Université Paul Sabatier of Toulouse, where she obtained her MSc degree in 2009. The last year of her MSc degree, she worked in the group of Dr Gourdon (Centre d'Élaboration de Matériaux et d'Etudes Structurales in Toulouse). Then, she continued to complete her $\mathrm{PhD}$ under his supervision and obtained a doctorate from Université Paul Sabatier in 2012. She has since moved to Paris and is currently working on human resources information systems.

André Gourdon is an engineer with a background at the ENSCP (Paris, 1975). After receiving a $\mathrm{PhD}$ in Chemistry in 1978 and working as a post-doc in Oxford (MLH Green's lab), he obtained a permanent position in 1981 as a CNRS Research Assistant and obtained a PhD in Physical Sciences (Doctorat d'Etat) in 1986. He then moved to the CEMES, Toulouse, France, in 1989 and became Research Director in 1999 in the NanoSciences Group (GNS-CEMES). His research interests include the design and synthesis of molecular devices for single-molecule experiments and molecular electronics.

\section{ACKNOWLEDGMENTS}

Financial support from the CNRS, the European Union (PAMS project, ICT FET grant 610446) and the ANR project ChimGraphN are gratefully acknowledged. AP is thankful to the 
French "Ministère de l'Enseignement Supérieur et de la Recherche" for a $\mathrm{PhD}$ grant. We gratefully acknowledge Prof. Kai Wu for Fig. 16.

\section{GLOSSARY}

2D

Two-dimensional

$3 \mathrm{D}$

Three-dimensional

$6 \mathrm{P}$

p-sexiphenyl

A

Hydrogen-bond acceptor

Ad

Adenine

AFM

Atomic force microscope/microscopy

ARPES

Angle-resolved photoelectron spectroscopy

BDA

(2,2'-bipyridine)-5,5'-dicarboxylic acid

BDATB

Phenyldiguanamine

BDG

Benzodiguanamine

BPB

Bis(4-pyridyl)-1,4-benzene

BPBP

4,4'-di(pyridin-4-yl)-1,1'-biphenyl

C

Cytosine

CA

Cyanuric acid

$\mathrm{CoPc}$

Cobalt(II) phthalocyanine

CPhDAT

6-(4'-cyanophenyl)-DAT

CT

Charge transfer

CuOEP

Copper(II) 2,3,7,8,12,13,17,18-octaethyl-21H,23H-porphyrine

$\mathrm{CuPc}$

Cu-phthalocyanine

CuTPP

Copper(II) tetraphenyl-21H,23H-porphirine 


\begin{tabular}{|c|c|}
\hline $\mathrm{D}$ & Hydrogen-bond donor \\
\hline DAT & 2,4-diamino-1,3,5-triazine \\
\hline DATP & 4,4''-diamino-p-terphenyl \\
\hline DFT & Density functional theory \\
\hline DCI & Dicarboxylic imide \\
\hline DIP & Diindeno[1,2,3-cd 1',2',3'-lm]perylene \\
\hline $\mathrm{F}_{14} \mathrm{Pen}$ & Perfluoropentacene \\
\hline $\mathrm{F}_{16} \mathrm{CuPc}$ & Fluorinated copper-phthalocyanine \\
\hline G & Guanine \\
\hline $\mathrm{HBC}$ & Hexa-peri-hexabenzocoronene \\
\hline H-bonding & Hydrogen-bonding \\
\hline HOPG & Highly oriented pyrolytic graphite \\
\hline HtBDC & Hexa-tert-butyl-decacyclene \\
\hline KMC & Kinetic Monte Carlo \\
\hline L-Phe-L-Phe & Di-L-phenylalanine \\
\hline M & Melamine (or 2,4,6-triamino-1,3,5-triazine) \\
\hline MC & Monte Carlo \\
\hline ML & Monolayer \\
\hline NC-AFM & Non-contact atomic force microscopy/microscope \\
\hline NEXAFS & Near-edge X-ray-absorption fine structure \\
\hline NTCDI & 1,4,5,8-naphthalene tetracarboxylic di-imide \\
\hline OEP & 2,3,7,8,12,13,17,18-octaethyl-21H,23H-porphine \\
\hline OFET & Organic field-effect transistor \\
\hline
\end{tabular}




\begin{tabular}{|c|c|}
\hline OLED & Organic light-emitting diode \\
\hline OPV & Organic photovoltaic \\
\hline PBP & 4,4'-bis(4-pyridyl)(2,2'-bipyrimidine \\
\hline $\mathrm{PcH}_{2}$ & Phthalocyanine \\
\hline PES & Photo-electron spectroscopy \\
\hline Pen & Pentacene \\
\hline PhDAT & Phenylguanamine (or 2,4-diamino-6-phenyl-1,3,5-triazine) \\
\hline PTBC & 1,3,5,7,9-penta-tert-butyl-corannulene \\
\hline PTCDA & 3,4,9,10-perylene tetracarboxylic di-anhydride \\
\hline PTCDI & 3,4,9,10-perylene tetracarboxylic diimide \\
\hline PTpy & (4',4'"'-(1,4-phenylene)bis-2,2'-6',2"-terpyridine) \\
\hline RT & Room temperature \\
\hline SANO & Self-assembly of nano-objects \\
\hline STM & Scanning tunneling microscopy/microscope \\
\hline STS & Scanning tunneling spectroscopy \\
\hline SubPc-Cl & Chloro[sub-phthalocyaninato]boron(III) \\
\hline $\mathrm{T}$ & Thymine \\
\hline TAB & 1,2,4,5-tetraaminobenzene \\
\hline TAPT & 2,4,6-tris(4-aminophenyl)-1,3,5-triazine \\
\hline $\mathrm{TPPH}_{2}$ & Tetraphenyl porphyrin \\
\hline TCNQ & Tetracyanoquinodimethane \\
\hline TDA & [1,1' 4',1"-terphenyl]-4,4"-dicarboxylic acid \\
\hline TMA & Trimesic acid \\
\hline
\end{tabular}


TNAP Tetracyanonaphthoquinodimethane

TPA Terephthalic acid

TPAD 1,3,5,7-tetraphenyladamantane

$\mathrm{TPPH}_{2} \quad 5,10,15,20$-tetraphenylporphyrin

$\mathrm{TPyPH}_{2} \quad 5,10,15,20$-tetra(4-pyridyl)porphyrin

TTF Tetrathiafulvalene

TTT Tetrathiatetracene

UHV Ultra-high vacuum

vdW $\quad$ van der Waals

XPS X-ray photoelectron spectroscopy

$\mathrm{ZnPcCl}_{18} \quad 2,3,9,10,16,17,23,24$-octachlorozinc phthalocyanine 


\section{REFERENCES}

(1) Dekens, O. Le Structuralisme; Armand Colin, 2015.

(2) Vicsek, T.; Zafeiris, A. Collective Motion. Phys. Rep. 2012, 517 (3-4), 71-140.

(3) Anderson, C.; Theraulaz, G.; Deneubourg, J.-L. Self-Assemblages in Insect Societies. Insectes Sociaux 2002, 49 (2), 99-110.

(4) Rubenstein, M.; Cornejo, A.; Nagpal, R. Programmable Self-Assembly in a ThousandRobot Swarm. Science 2014, 345 (6198), 795-799.

(5) Volfson, D.; Cookson, S.; Hasty, J.; Tsimring, L. S. Biomechanical Ordering of Dense Cell Populations. Proc. Natl. Acad. Sci. 2008, 105 (40), 15346-15351.

(6) Copeland, M. F.; Weibel, D. B. Bacterial Swarming: A Model System for Studying Dynamic Self-Assembly. Soft Matter 2009, 5 (6), 1174-1187.

(7) Alonso, J. M.; Górzny, M. Ł.; Bittner, A. M. The Physics of Tobacco Mosaic Virus and Virus-Based Devices in Biotechnology. Trends Biotechnol. 2013, 31 (9), 530-538.

(8) Pouget, E.; Dujardin, E.; Cavalier, A.; Moreac, A.; Valéry, C.; Marchi-Artzner, V.; Weiss, T.; Renault, A.; Paternostre, M.; Artzner, F. Hierarchical Architectures by Synergy between Dynamical Template Self-Assembly and Biomineralization. Nat. Mater. 2007, 6 (6), 434-439.

(9) Valéry, C.; Artzner, F.; Paternostre, M. Peptide Nanotubes: Molecular Organisations, Self-Assembly Mechanisms and Applications. Soft Matter 2011, 7 (20), 9583-9594.

(10) Hamley, I. W. Peptide Nanotubes. Angew. Chem. Int. Ed. 2014, 53 (27), 6866-6881.

(11) Rothemund, P. W. K. Folding DNA to Create Nanoscale Shapes and Patterns. Nature 2006, 440 (7082), 297-302.

(12) Poirier, G. E. Characterization of Organosulfur Molecular Monolayers on Au (111) Using Scanning Tunneling Microscopy. Chem. Rev. 1997, 97 (4), 1117-1128.

(13) De Feyter, S.; Gesquière, A.; Abdel-Mottaleb, M. M.; Grim, P. C. M.; De Schryver, F. C.; Meiners, C.; Sieffert, M.; Valiyaveettil, S.; Müllen, K. Scanning Tunneling Microscopy: A Unique Tool in the Study of Chirality, Dynamics, and Reactivity in Physisorbed Organic Monolayers. Acc. Chem. Res. 2000, 33 (8), 520-531.

(14) De Feyter, S.; De Schryver, F. C. Two-Dimensional Supramolecular Self-Assembly Probed by Scanning Tunneling Microscopy. Chem. Soc. Rev. 2003, 32 (3), 139-150.

(15) Elemans, J. a. a. W.; van Hameren, R.; Nolte, R. J. M.; Rowan, A. E. Molecular Materials by Self-Assembly of Porphyrins, Phthalocyanines, and Perylenes. Adv. Mater. 2006, 18 (10), $1251-1266$.

(16) Wan, L.-J. Fabricating and Controlling Molecular Self-Organization at Solid Surfaces: Studies by Scanning Tunneling Microscopy. Acc. Chem. Res. 2006, 39 (5), 334-342. 
(17) Bonifazi, D.; Enger, O.; Diederich, F. Supramolecular [60]fullerene Chemistry on Surfaces. Chem. Soc. Rev. 2007, 36 (2), 390-414.

(18) Gomar-Nadal, E.; Puigmartí-Luis, J.; Amabilino, D. B. Assembly of Functional Molecular Nanostructures on Surfaces. Chem. Soc. Rev. 2008, 37 (3), 490-504.

(19) Whitelam, S. Examples of Molecular Self-Assembly at Surfaces. Adv. Mater. 2015, 27 (38), 5720-5725.

(20) Hoeben, F. J. M.; Jonkheijm, P.; Meijer, E. W.; Schenning, A. P. H. J. About Supramolecular Assemblies of $\pi$-Conjugated Systems. Chem. Rev. 2005, 105 (4), 1491-1546.

(21) Ohtani, H.; Wilson, R. J.; Chiang, S.; Mate, C. M. Scanning Tunneling Microscopy Observations of Benzene Molecules on the Rh(111)-(3 × 3) ( C $6 \mathrm{H} 6+2 \mathrm{CO})$ Surface. Phys. Rev. Lett. 1988, 60 (23), 2398-2401.

(22) De Feyter, S.; De Schryver, F. C. Self-Assembly at the Liquid/Solid Interface: STM Reveals. J. Phys. Chem. B 2005, 109 (10), 4290-4302.

(23) Pinheiro, L. S.; Temperini, M. L. A. Coadsorption of 2-Mercaptopyrimidine and 1,10'Phenanthroline on $\mathrm{Au}(111)$ as Seen by STM. Surf. Sci. 1999, 441 (1), 53-64.

(24) Lei, S.; Surin, M.; Tahara, K.; Adisoejoso, J.; Lazzaroni, R.; Tobe, Y.; Feyter, S. D. Programmable Hierarchical Three-Component 2D Assembly at a Liquid-Solid Interface: Recognition, Selection, and Transformation. Nano Lett. 2008, 8 (8), 2541-2546.

(25) Tahara, K.; Gotoda, J.; Carroll, C. N.; Hirose, K.; De Feyter, S.; Tobe, Y. Square Tiling by Square Macrocycles at the Liquid/Solid Interface: Co-Crystallisation with One- or TwoDimensional Order. Chem. - Eur. J. 2015, 21 (18), 6806-6816.

(26) Ghijsens, E.; Adisoejoso, J.; Van Gorp, H.; Destoop, I.; Noguchi, A.; Ivasenko, O.; Tahara, K.; Van der Auweraer, M.; Tobe, Y.; De Feyter, S. On the Stability of Surface-Confined Nanoporous Molecular Networks. J. Chem. Phys. 2015, 142 (10), 101932.

(27) Balandina, T.; Tahara, K.; Sändig, N.; Blunt, M. O.; Adisoejoso, J.; Lei, S.; Zerbetto, F.; Tobe, Y.; De Feyter, S. Role of Substrate in Directing the Self-Assembly of Multicomponent Supramolecular Networks at the Liquid-Solid Interface. ACS Nano 2012, 6 (9), 8381-8389.

(28) Dong, W.; Wang, L.; Ding, H.; Zhao, L.; Wang, D.; Wang, C.; Wan, L.-J. Substrate Orientation Effect in the On-Surface Synthesis of Tetrathiafulvalene-Integrated Single-Layer Covalent Organic Frameworks. Langmuir 2015, 31 (43), 11755-11759.

(29) Jahanbekam, A.; Chilukuri, B.; Mazur, U.; Hipps, K. W. Kinetically Trapped TwoComponent Self-Assembled Adlayer. J. Phys. Chem. C 2015, 119 (45), 25364-25376.

(30) Stadtmüller, B.; Gruenewald, M.; Peuker, J.; Forker, R.; Fritz, T.; Kumpf, C. Molecular Exchange in a Heteromolecular PTCDA/CuPc Bilayer Film on $\operatorname{Ag}(111)$. J. Phys. Chem. C 2014, 118 (49), 28592-28602. 
(31) Korolkov, V. V.; Allen, S.; Roberts, C. J.; Tendler, S. J. B. Green Chemistry Approach to Surface Decoration: Trimesic Acid Self-Assembly on HOPG. J. Phys. Chem. C 2012, 116 (21), $11519-11525$.

(32) Cebula, I.; Räisänen, M. T.; Madueno, R.; Karamzadeh, B.; Buck, M. Comment on “The Structure and Formation of Hydrogen-Bonded Molecular Networks on Au(111) Surfaces Revealed by Scanning Tunnelling and Torsional-Tapping Atomic Force Microscopy" by V. V. Korolkov, N. Mullin, S. Allen, C. J. Roberts, J. K. Hobbs and S. J. B. Tendler, Phys. Chem. Chem. Phys., 2012, 14, 15909. Phys. Chem. Chem. Phys. 2013, 15 (33), 14126.

(33) Korolkov, V. V.; Mullin, N.; Allen, S.; Roberts, C. J.; Hobbs, J. K.; Tendler, S. J. B. The Structure and Formation of Hydrogen-Bonded Molecular Networks on Au(111) Surfaces Revealed by Scanning Tunnelling and Torsional-Tapping Atomic Force Microscopy. Phys. Chem. Chem. Phys. 2012, 14 (45), 15909-15916.

(34) Korolkov, V. V.; Mullin, N.; Allen, S.; Roberts, C. J.; Hobbs, J. K.; Tendler, S. J. B. Reply to the "Comment on 'The Structure and Formation of Hydrogen-Bonded Molecular Networks on Au(111) Surfaces Revealed by Scanning Tunnelling and Torsional-Tapping Atomic Force Microscopy"' by I. Cebula, M. T. Räisänen, R. Madueno, B. Karamzadeh and M. Buck, Phys. Chem. Chem. Phys., 2013, 15, DOI: 10.1039/c3cp50754h. Phys. Chem. Chem. Phys. 2013, 15 (33), 14128-14129.

(35) Rauschenbach, S.; Vogelgesang, R.; Malinowski, N.; Gerlach, J. W.; Benyoucef, M.; Costantini, G.; Deng, Z.; Thontasen, N.; Kern, K. Electrospray Ion Beam Deposition: SoftLanding and Fragmentation of Functional Molecules at Solid Surfaces. ACS Nano 2009, 3 (10), 2901-2910.

(36) Rauschenbach, S.; Stadler, F. L.; Lunedei, E.; Malinowski, N.; Koltsov, S.; Costantini, G.; Kern, K. Electrospray Ion Beam Deposition of Clusters and Biomolecules. Small 2006, 2 (4), 540-547.

(37) Saywell, A.; Magnano, G.; Satterley, C. J.; Perdigão, L. M. A.; Champness, N. R.; Beton, P. H.; O'Shea, J. N. Electrospray Deposition of C60 on a Hydrogen-Bonded Supramolecular Network. J. Phys. Chem. C 2008, 112 (20), 7706-7709.

(38) Saywell, A.; Magnano, G.; Satterley, C. J.; Perdigão, L. M. A.; Britton, A. J.; Taleb, N.; del Carmen Giménez-López, M.; Champness, N. R.; O’Shea, J. N.; Beton, P. H. Self-Assembled Aggregates Formed by Single-Molecule Magnets on a Gold Surface. Nat. Commun. 2010, 1 (6), $1-8$.

(39) Saywell, A.; Sprafke, J. K.; Esdaile, L. J.; Britton, A. J.; Rienzo, A.; Anderson, H. L.; O'Shea, J. N.; Beton, P. H. Conformation and Packing of Porphyrin Polymer Chains Deposited Using Electrospray on a Gold Surface. Angew. Chem. Int. Ed. 2010, 49 (48), 9136-9139.

(40) Nair, M. N.; Mattioli, C.; Cranney, M.; Malval, J.-P.; Vonau, F.; Aubel, D.; Bubendorff, J.-L.; Gourdon, A.; Simon, L. STM Studies of Self-Assembled Tetrathiafulvalene (TTF) Derivatives on Graphene: Influence of the Mode of Deposition. J. Phys. Chem. C 2015, 119 (17), 9334-9341. 
(41) Inokuchi, H.; Saito, G.; Wu, P.; Seki, K.; Tang, T. B.; Mori, T.; Imaeda, K.; Enoki, T.; Higuchi, Y.; Inaka, K.; et al. A Novel Type of Organic Semiconductors. Molecular Fastener. Chem. Lett. 1986, 15 (8), 1263-1266.

(42) Dediu, V. A.; Hueso, L. E.; Bergenti, I.; Taliani, C. Spin Routes in Organic Semiconductors. Nat. Mater. 2009, 8 (9), 707-716.

(43) Rivnay, J.; Mannsfeld, S. C. B.; Miller, C. E.; Salleo, A.; Toney, M. F. Quantitative Determination of Organic Semiconductor Microstructure from the Molecular to Device Scale. Chem. Rev. 2012, 112 (10), 5488-5519.

(44) Xue, J.; Rand, B. P.; Uchida, S.; Forrest, S. R. A Hybrid Planar-Mixed Molecular Heterojunction Photovoltaic Cell. Adv. Mater. 2005, 17 (1), 66-71.

(45) Cicoira, F.; Santato, C.; Rosei, F. Two-Dimensional Nanotemplates as Surface Cues for the Controlled Assembly of Organic Molecules. In STM and AFM Studies on (Bio)molecular Systems: Unravelling the Nanoworld; Samorì, P., Ed.; Topics in Current Chemistry; Springer Berlin Heidelberg, 2008; pp 203-267.

(46) Bonifazi, D.; Mohnani, S.; Llanes-Pallas, A. Supramolecular Chemistry at Interfaces: Molecular Recognition on Nanopatterned Porous Surfaces. Chem. - Eur. J. 2009, 15 (29), 70047025 .

(47) Cui, K.; Schlütter, F.; Ivasenko, O.; Kivala, M.; Schwab, M. G.; Lee, S.-L.; Mertens, S. F. L.; Tahara, K.; Tobe, Y.; Müllen, K.; et al. Multicomponent Self-Assembly with a ShapePersistent N-Heterotriangulene Macrocycle on Au(111). Chem. - Eur. J. 2015, 21 (4), 16521659.

(48) Adisoejoso, J.; Tahara, K.; Lei, S.; Szabelski, P.; Rżysko, W.; Inukai, K.; Blunt, M. O.; Tobe, Y.; De Feyter, S. One Building Block, Two Different Nanoporous Self-Assembled Monolayers: A Combined STM and Monte Carlo Study. ACS Nano 2012, 6 (1), 897-903.

(49) Banerjee, K.; Kumar, A.; Canova, F. F.; Kezilebieke, S.; Foster, A. S.; Liljeroth, P. Flexible Self-Assembled Molecular Templates on Graphene. J. Phys. Chem. C 2016, 120 (16), 8772-8780.

(50) Stadtmüller, B.; Schröder, S.; Kumpf, C. Heteromolecular Metal-organic Interfaces: Electronic and Structural Fingerprints of Chemical Bonding. J. Electron Spectrosc. Relat. Phenom. 2015, 204, 80-91.

(51) Ulman, A. Formation and Structure of Self-Assembled Monolayers. Chem. Rev. 1996, 96 (4), 1533-1554.

(52) Forrest, S. R. Ultrathin Organic Films Grown by Organic Molecular Beam Deposition and Related Techniques. Chem. Rev. 1997, 97 (6), 1793-1896.

(53) Service, R. F.; Szuromi, P.; Uppenbrink, J. Strength in Numbers. Science 2002, 295 (5564), 2395-2395.

(54) Halpern, J. Reaching across the Sciences. Proc. Natl. Acad. Sci. 2002, 99 (8), 4762-4762. 
(55) Barth, J. V.; Costantini, G.; Kern, K. Engineering Atomic and Molecular Nanostructures at Surfaces. Nature 2005, 437 (7059), 671-679.

(56) Barth, J. V. Molecular Architectonic on Metal Surfaces. Annu. Rev. Phys. Chem. 2007, $58(1), 375-407$.

(57) Ma, X.; Yang, Y.; Deng, K.; Zeng, Q.; Zhao, K.; Wang, C.; Bai, C. Molecular Miscibility Characteristics of Self-Assembled 2D Molecular Architectures. J. Mater. Chem. 2008, 18 (18), 2074-2081.

(58) Kühnle, A. Self-Assembly of Organic Molecules at Metal Surfaces. Curr. Opin. Colloid Interface Sci. 2009, 14 (2), 157-168.

(59) Lin, N.; Stepanow, S.; Ruben, M.; Barth, J. V. Surface-Confined Supramolecular Coordination Chemistry. In Templates in Chemistry III; Broekmann, P., Dötz, K.-H., Schalley, C. A., Eds.; Springer Berlin Heidelberg: Berlin, Heidelberg, 2008; Vol. 287, pp 1-44.

(60) Bartels, L. Tailoring Molecular Layers at Metal Surfaces. Nat. Chem. 2010, 2 (2), 87-95.

(61) Koepf, M.; Weiss, J.; Cherioux, F.; Wytko, J. A. 1D and 3D Surface-Assisted SelfOrganization. Coord. Chem. Rev. 2012.

(62) Claridge, S. A.; Liao, W.-S.; Thomas, J. C.; Zhao, Y.; Cao, H. H.; Cheunkar, S.; Serino, A. C.; Andrews, A. M.; Weiss, P. S. From the Bottom up: Dimensional Control and Characterization in Molecular Monolayers. Chem. Soc. Rev. 2013, 42 (7), 2725-2745.

(63) Goiri, E.; Borghetti, P.; El-Sayed, A.; Ortega, J. E.; de Oteyza, D. G. Multi-Component Organic Layers on Metal Substrates. Adv. Mater. 2016, 28 (7), 1340-1368.

(64) Surin, M.; Samorì, P. Multicomponent Monolayer Architectures at the Solid-Liquid Interface: Towards Controlled Space-Confined Properties and Reactivity of Functional Building Blocks. Small 2007, 3 (2), 190-194.

(65) Finnis, M. Interatomic Forces in Condensed Matter, Oxford University Press.; Oxford series on materials modelling; Adrian P. Sutton and Robert E. Rudd: Oxford, 2003.

(66) Stone, A. The Theory of Intermolecular Forces, Second Edition.; Oxford University Press: Oxford, 2013.

(67) Girard, C.; Bouju, X.; Joachim, C. Van Der Waals Interactions between an Adsorbate and the Tip of an STM. Chem. Phys. 1992, 168 (2), 203-210.

(68) Gravil, P. A.; Devel, M.; Lambin, P.; Bouju, X.; Girard, C.; Lucas, A. A. Adsorption of C_\{60\} Molecules. Phys. Rev. B 1996, 53 (3), 1622-1629.

(69) Yang, Y.; Wang, C. Hierarchical Construction of Self-Assembled Low-Dimensional Molecular Architectures Observed by Using Scanning Tunneling Microscopy. Chem. Soc. Rev. 2009, 38 (9), 2576.

(70) Israelachvili, J. N. Intermolecular and Surface Forces; Academic Press Inc. (London), 1992. 
(71) Gourdon, A. Synthesis of "Molecular Landers." Eur. J. Org. Chem. 1998, 1998 (12), 2797-2801.

(72) Zambelli, T.; Goudeau, S.; Lagoute, J.; Gourdon, A.; Bouju, X.; Gauthier, S. Molecular Self-Assembly of Jointed Molecules on a Metallic Substrate: From Single Molecule to Monolayer. ChemPhysChem 2006, 7 (9), 1917-1920.

(73) Guillermet, O.; Niemi, E.; Nagarajan, S.; Bouju, X.; Martrou, D.; Gourdon, A.; Gauthier, S. Self-Assembly of Fivefold-Symmetric Molecules on a Threefold-Symmetric Surface. Angew. Chem. Int. Ed. 2009, 48 (11), 1970-1973.

(74) Ghijsens, E.; Ivasenko, O.; Tahara, K.; Yamaga, H.; Itano, S.; Balandina, T.; Tobe, Y.; De Feyter, S. A Tale of Tails: Alkyl Chain Directed Formation of 2D Porous Networks Reveals Odd-Even Effects and Unexpected Bicomponent Phase Behavior. ACS Nano 2013, 7 (9), 80318042.

(75) Yin, S.; Wang, C.; Qiu, X.; Xu, B.; Bai, C. Theoretical Study of the Effects of Intermolecular Interactions in Self-Assembled Long-Chain Alkanes Adsorbed on Graphite Surface. Surf. Interface Anal. 2001, 32 (1), 248-252.

(76) Chen, Q.; Chen, T.; Pan, G.-B.; Yan, H.-J.; Song, W.-G.; Wan, L.-J.; Li, Z.-T.; Wang, Z.H.; Shang, B.; Yuan, L.-F. Structural Selection of Graphene Supramolecular Assembly Oriented by Molecular Conformation and Alkyl Chain. Proc. Natl. Acad. Sci. 2008, 105 (44), 1684916854.

(77) Gong, J.-R.; Wan, L.-J.; Yuan, Q.-H.; Bai, C.-L.; Jude, H.; Stang, P. J. Mesoscopic SelfOrganization of a Self-Assembled Supramolecular Rectangle on Highly Oriented Pyrolytic Graphite and Au(111) Surfaces. Proc. Natl. Acad. Sci. U. S. A. 2005, 102 (4), 971-974.

(78) Arunan, E.; Desiraju, G. R.; Klein, R. A.; Sadlej, J.; Scheiner, S.; Alkorta, I.; Clary, D. C.; Crabtree, R. H.; Dannenberg, J. J.; Hobza, P.; et al. Definition of the Hydrogen Bond (IUPAC Recommendations 2011). Pure Appl. Chem. 2011, 83 (8), 1637-1641.

(79) Arunan, E.; Desiraju, G. R.; Klein, R. A.; Sadlej, J.; Scheiner, S.; Alkorta, I.; Clary, D. C.; Crabtree, R. H.; Dannenberg, J. J.; Hobza, P.; et al. Defining the Hydrogen Bond: An Account (IUPAC Technical Report). Pure Appl. Chem. 2011, 83 (8), 1619-1636.

(80) Jeffrey, G. A. An Introduction to Hydrogen Bonding; Oxford University Press, 1997.

(81) Desiraju, G. R. Chemistry beyond the Molecule. Nature 2001, 412 (6845), 397-400.

(82) Desiraju, G. R. A Bond by Any Other Name. Angew. Chem. Int. Ed. 2011, 50 (1), 52-59.

(83) Desiraju, G. R. Crystal Engineering: From Molecule to Crystal. J. Am. Chem. Soc. 2013, 135 (27), 9952-9967.

(84) Steiner, T. The Hydrogen Bond in the Solid State. Angew. Chem. Int. Ed. 2002, 41 (1), 48-76. 
(85) Prins, L. J.; Reinhoudt, D. N.; Timmerman, P. Noncovalent Synthesis Using Hydrogen Bonding. Angew. Chem. Int. Ed. 2001, 40 (13), 2382-2426.

(86) Yokoyama, T.; Yokoyama, S.; Kamikado, T.; Okuno, Y.; Mashiko, S. Selective Assembly on a Surface of Supramolecular Aggregates with Controlled Size and Shape. Nature 2001, 413 (6856), 619-621.

(87) Wong, K. L.; Pawin, G.; Kwon, K.-Y.; Lin, X.; Jiao, T.; Solanki, U.; Fawcett, R. H. J.; Bartels, L.; Stolbov, S.; Rahman, T. S. A Molecule Carrier. Science 2007, 315 (5817), 13911393.

(88) Nishio, M. The $\mathrm{CH} / \pi$ Hydrogen Bond in Chemistry. Conformation, Supramolecules, Optical Resolution and Interactions Involving Carbohydrates. Phys. Chem. Chem. Phys. 2011, 13 (31), 13873-13900.

(89) Tsuzuki, S.; Honda, K.; Uchimaru, T.; Mikami, M.; Tanabe, K. Origin of the Attraction and Directionality of the $\mathrm{NH} / \pi$ Interaction: Comparison with $\mathrm{OH} / \pi$ and $\mathrm{CH} / \pi$ Interactions. $J$. Am. Chem. Soc. 2000, 122 (46), 11450-11458.

(90) Plevin, M. J.; Bryce, D. L.; Boisbouvier, J. Direct Detection of $\mathrm{CH} / \pi$ Interactions in Proteins. Nat. Chem. 2010, 2 (6), 466-471.

(91) Lii, J.-H.; Allinger, N. L. Directional Hydrogen Bonding in the MM3 Force Field: II. J. Comput. Chem. 1998, 19 (9), 1001-1016.

(92) Allinger, N. L. Understanding Molecular Structure from Molecular Mechanics. J. Comput. Aided Mol. Des. 2011, 25, 295-316.

(93) Mayo, S. L.; Olafson, B. D.; Goddard, W. A. DREIDING: A Generic Force Field for Molecular Simulations. J. Phys. Chem. 1990, 94 (26), 8897-8909.

(94) Sun, H.; Mumby, S. J.; Maple, J. R.; Hagler, A. T. An Ab Initio CFF93 All-Atom Force Field for Polycarbonates. J. Am. Chem. Soc. 1994, 116 (7), 2978-2987.

(95) Sun, H. Force Field for Computation of Conformational Energies, Structures, and Vibrational Frequencies of Aromatic Polyesters. J. Comput. Chem. 1994, 15 (7), 752-768.

(96) Cornell, W. D.; Cieplak, P.; Bayly, C. I.; Gould, I. R.; Merz, K. M.; Ferguson, D. M.; Spellmeyer, D. C.; Fox, T.; Caldwell, J. W.; Kollman, P. A. A Second Generation Force Field for the Simulation of Proteins, Nucleic Acids, and Organic Molecules. J. Am. Chem. Soc. 1995, 117 (19), 5179-5197.

(97) Case, D. A.; Cheatham, T. E.; Darden, T.; Gohlke, H.; Luo, R.; Merz, K. M.; Onufriev, A.; Simmerling, C.; Wang, B.; Woods, R. J. The Amber Biomolecular Simulation Programs. J. Comput. Chem. 2005, 26 (16), 1668-1688.

(98) MacKerell, A. D.; Banavali, N.; Foloppe, N. Development and Current Status of the CHARMM Force Field for Nucleic Acids. Biopolymers 2000, 56 (4), 257-265. 
(99) Boese, A. D. Assessment of Coupled Cluster Theory and More Approximate Methods for Hydrogen Bonded Systems. J. Chem. Theory Comput. 2013, 9 (10), 4403-4413.

(100) Ayoub, A. T.; Tuszynski, J.; Klobukowski, M. Estimating Hydrogen Bond Energies: Comparison of Methods. Theor. Chem. Acc. 2014, 133 (8), 1-7.

(101) Boese, A. D. Density Functional Theory and Hydrogen Bonds: Are We There Yet? ChemPhysChem 2015, 16 (5), 978-985.

(102) Martsinovich, N.; Troisi, A. Modeling the Self-Assembly of Benzenedicarboxylic Acids Using Monte Carlo and Molecular Dynamics Simulations. J. Phys. Chem. C 2010, 114 (10), 4376-4388.

(103) Kelly, R. E. A.; Xu, W.; Lukas, M.; Otero, R.; Mura, M.; Lee, Y.-J.; Laegsgaard, E.; Stensgaard, I.; Kantorovich, L. N.; Besenbacher, F. An Investigation into the Interactions Between Self-Assembled Adenine Molecules and a Au(111) Surface. Small 2008, 4 (9), 14941500 .

(104) Aakeröy, C. B.; Fasulo, M.; Schultheiss, N.; Desper, J.; Moore, C. Structural Competition between Hydrogen Bonds and Halogen Bonds. J Am Chem Soc 2011, 129 (45), 13772-13773.

(105) Voth, A. R.; Khuu, P.; Oishi, K.; Ho, P. S. Halogen Bonds as Orthogonal Molecular Interactions to Hydrogen Bonds. Nat. Chem. 2009, 1 (1), 74-79.

(106) Priimagi, A.; Cavallo, G.; Metrangolo, P.; Resnati, G. The Halogen Bond in the Design of Functional Supramolecular Materials: Recent Advances. Acc. Chem. Res. 2013, 46 (11), 2686-2695.

(107) Gilday, L. C.; Robinson, S. W.; Barendt, T. A.; Langton, M. J.; Mullaney, B. R.; Beer, P. D. Halogen Bonding in Supramolecular Chemistry. Chem. Rev. 2015, 115 (15), 7118-7195.

(108) Priimagi, A.; Cavallo, G.; Metrangolo, P.; Resnati, G. The Halogen Bond in the Design of Functional Supramolecular Materials: Recent Advances. Acc. Chem. Res. 2013, 46 (11), 2686-2695.

(109) Cavallo, G.; Metrangolo, P.; Milani, R.; Pilati, T.; Priimagi, A.; Resnati, G.; Terraneo, G. The Halogen Bond. Chem. Rev. 2016, 116 (4), 2478-2601.

(110) Chung, K.-H.; Park, J.; Kim, K. Y.; Yoon, J. K.; Kim, H.; Han, S.; Kahng, S.-J. Polymorphic Porous Supramolecular Networks Mediated by Halogen Bonds on Ag(111). Chem Commun 2011, 47 (41), 11492-11494.

(111) El Garah, M.; Lipton-Duffin, J.; MacLeod, J. M.; Gutzler, R.; Palmino, F.; Luzet, V.; Chérioux, F.; Rosei, F. Self-Assembly of a Halogenated Molecule on Oxide-Passivated Cu(110). Chem. - Asian J. 2013, 8 (8), 1813-1817.

(112) Walch, H.; Gutzler, R.; Sirtl, T.; Eder, G.; Lackinger, M. Material- and OrientationDependent Reactivity for Heterogeneously Catalyzed Carbon-Bromine Bond Homolysis. J. Phys. Chem. C 2010, 114 (29), 12604-12609. 
(113) Grill, L.; Dyer, M.; Lafferentz, L.; Persson, M.; Peters, M. V.; Hecht, S. NanoArchitectures by Covalent Assembly of Molecular Building Blocks. Nat. Nanotechnol. 2007, 2 (11), 687-691.

(114) Kawai, S.; Sadeghi, A.; Xu, F.; Peng, L.; Orita, A.; Otera, J.; Goedecker, S.; Meyer, E. Extended Halogen Bonding between Fully Fluorinated Aromatic Molecules. ACS Nano 2015, 9 (3), 2574-2583.

(115) Gutzler, R.; Ivasenko, O.; Fu, C.; Brusso, J. L.; Rosei, F.; Perepichka, D. F. Halogen Bonds as Stabilizing Interactions in a Chiral Self-Assembled Molecular Monolayer. Chem. Commun. 2011, 47 (33), 9453.

(116) Gutzler, R.; Fu, C.; Dadvand, A.; Hua, Y.; MacLeod, J. M.; Rosei, F.; Perepichka, D. F. Halogen Bonds in 2D Supramolecular Self-Assembly of Organic Semiconductors. Nanoscale 2012, 4 (19), 5965.

(117) Silly, F. Selecting Two-Dimensional Halogen-Halogen Bonded Self-Assembled 1,3,5Tris(4-Iodophenyl)benzene Porous Nanoarchitectures at the Solid-Liquid Interface. J. Phys. Chem. C 2013, 117 (39), 20244-20249.

(118) Baris, B.; Luzet, V.; Duverger, E.; Sonnet, P.; Palmino, F.; Cherioux, F. Robust and Open Tailored Supramolecular Networks Controlled by the Template Effect of a Silicon Surface. Angew. Chem. Int. Ed. 2011, 50 (18), 4094-4098.

(119) Hunter, C. A.; Lawson, K. R.; Perkins, J.; Urch, C. J. Aromatic Interactions. J. Chem. Soc. Perkin Trans. 2 2001, No. 5, 651-669.

(120) Meyer, E. A.; Castellano, R. K.; Diederich, F. Interactions with Aromatic Rings in Chemical and Biological Recognition. Angew. Chem. Int. Ed. 2003, 42 (11), 1210-1250.

(121) Hunter, C. A.; Sanders, J. K. M. The Nature of .pi.-.pi. Interactions. J. Am. Chem. Soc. 1990,112 (14), 5525-5534.

(122) Hunter, C. A.; Singh, J.; Thornton, J. M. $\pi-\pi$ Interactions: The Geometry and Energetics of Phenylalanine-Phenylalanine Interactions in Proteins. J. Mol. Biol. 1991, 218 (4), 837-846.

(123) Tsuzuki, S.; Honda, K.; Uchimaru, T.; Mikami, M.; Tanabe, K. Origin of Attraction and Directionality of the $\pi / \pi$ Interaction: Model Chemistry Calculations of Benzene Dimer Interaction. J. Am. Chem. Soc. 2002, 124 (1), 104-112.

(124) Sinnokrot, M. O.; Valeev, E. F.; Sherrill, C. D. Estimates of the Ab Initio Limit for $\Pi-\pi$ Interactions: The Benzene Dimer. J. Am. Chem. Soc. 2002, 124 (36), 10887-10893.

(125) Sinnokrot, M. O.; Sherrill, C. D. Substituent Effects in $\Pi-\pi$ Interactions: Sandwich and T-Shaped Configurations. J. Am. Chem. Soc. 2004, 126 (24), 7690-7697.

(126) Sinnokrot, M. O.; Sherrill, C. D. Highly Accurate Coupled Cluster Potential Energy Curves for the Benzene Dimer: Sandwich, T-Shaped, and Parallel-Displaced Configurations. $J$. Phys. Chem. A 2004, 108 (46), 10200-10207. 
(127) Sinnokrot, M. O.; Sherrill, C. D. High-Accuracy Quantum Mechanical Studies of $\Pi-\pi$ Interactions in Benzene Dimers. J. Phys. Chem. A 2006, 110 (37), 10656-10668.

(128) Sherrill, C. D.; Takatani, T.; Hohenstein, E. G. An Assessment of Theoretical Methods for Nonbonded Interactions: Comparison to Complete Basis Set Limit Coupled-Cluster Potential Energy Curves for the Benzene Dimer, the Methane Dimer, Benzene-Methane, and Benzene-H2S†. J. Phys. Chem. A 2009, 113 (38), 10146-10159.

(129) Parschau, M.; Fasel, R.; Ernst, K.; Gröning, O.; Brandenberger, L.; Schillinger, R.; Greber, T.; Seitsonen, A. P.; Wu, Y.; Siegel, J. S. Buckybowls on Metal Surfaces: Symmetry Mismatch and Enantiomorphism of Corannulene on $\mathrm{Cu}(110)$. Angew. Chem. Int. Ed. 2007, 46 (43), 8258-8261.

(130) Maier, S.; Fendt, L.-A.; Zimmerli, L.; Glatzel, T.; Pfeiffer, O.; Diederich, F.; Meyer, E. Nanoscale Engineering of Molecular Porphyrin Wires on Insulating Surfaces. Small 2008, 4 (8), 1115-1118.

(131) Shi, X. Q.; Lin, C.; Minot, C.; Tseng, T.-C.; Tait, S. L.; Lin, N.; Zhang, R. Q.; Kern, K.; Cerdá, J. I.; Van Hove, M. A. Structural Analysis and Electronic Properties of Negatively Charged TCNQ: 2D Networks of (TCNQ) 2 Mn Assembled on $\mathrm{Cu}(100)^{\dagger}$. J. Phys. Chem. C 2010, 114 (40), 17197-17204.

(132) Beverina, L.; Pagani, G. A. $\pi$-Conjugated Zwitterions as Paradigm of Donor-Acceptor Building Blocks in Organic-Based Materials. Acc. Chem. Res. 2014, 47 (2), 319-329.

(133) Nony, L.; Bocquet, F.; Para, F.; Chérioux, F.; Duverger, E.; Palmino, F.; Luzet, V.; Loppacher, C. Dipole-Driven Self-Organization of Zwitterionic Molecules on Alkali Halide Surfaces. Beilstein J. Nanotechnol. 2012, 3, 285-293.

(134) Schiffrin, A.; Riemann, A.; Auwärter, W.; Pennec, Y.; Weber-Bargioni, A.; Cvetko, D.; Cossaro, A.; Morgante, A.; Barth, J. V. Zwitterionic Self-Assembly of L-Methionine Nanogratings on the Ag(111) Surface. Proc. Natl. Acad. Sci. 2007, 104 (13), 5279-5284.

(135) Reichert, J.; Schiffrin, A.; Auwärter, W.; Weber-Bargioni, A.; Marschall, M.; Dell'Angela, M.; Cvetko, D.; Bavdek, G.; Cossaro, A.; Morgante, A.; et al. L-Tyrosine on $\operatorname{Ag}(111)$ : Universality of the Amino Acid 2D Zwitterionic Bonding Scheme? ACS Nano 2010, 4 (2), 1218-1226.

(136) Makoudi, Y.; Arab, M.; Palmino, F.; Duverger, E.; Ramseyer, C.; Picaud, F.; Chérioux, F. A Stable Room-Temperature Molecular Assembly of Zwitterionic Organic Dipoles Guided by a Si(111)-7×7 Template Effect. Angew. Chem. Int. Ed. 2007, 46 (48), 9287-9290.

(137) Makoudi, Y.; Garah, M. E.; Palmino, F.; Duverger, E.; Arab, M.; Cherioux, F. Adsorption of an Organic Zwitterion on a Si(111)-7×7 Surface at Room Temperature. Surf. Sci. 2008, 602 (16), 2719-2723.

(138) El Garah, M.; Makoudi, Y.; Duverger, E.; Palmino, F.; Rochefort, A.; Chérioux, F. Large-Scale Patterning of Zwitterionic Molecules on a Si(111)-7 × 7 Surface. ACS Nano 2011, 5 (1), 424-428. 
(139) El Garah, M.; Palmino, F.; Chérioux, F.; Melinte, S.; Hackens, B.; Rodrigues, M. S.; Bogdan, D.; Duverger, E. Adsorption of Zwitterionic Assemblies on Si(111)-7 × 7: A Joint Tunneling Spectroscopy and Ab Initio Study. Phys. Rev. B 2012, 85 (3), 35425.

(140) Cabellos, J. L.; Mowbray, D. J.; Goiri, E.; El-Sayed, A.; Floreano, L.; de Oteyza, D. G.; Rogero, C.; Ortega, J. E.; Rubio, A. Understanding Charge Transfer in Donor-Acceptor/Metal Systems: A Combined Theoretical and Experimental Study. J. Phys. Chem. C 2012, 116 (34), 17991-18001.

(141) El-Sayed, A.; Borghetti, P.; Goiri, E.; Rogero, C.; Floreano, L.; Lovat, G.; Mowbray, D. J.; Cabellos, J. L.; Wakayama, Y.; Rubio, A.; et al. Understanding Energy-Level Alignment in Donor-Acceptor/Metal Interfaces from Core-Level Shifts. ACS Nano 2013, 7 (8), 6914-6920.

(142) Zhong, J.-Q.; Qin, X.; Zhang, J.-L.; Kera, S.; Ueno, N.; Wee, A. T. S.; Yang, J.; Chen, W. Energy Level Realignment in Weakly Interacting Donor-Acceptor Binary Molecular Networks. ACS Nano 2014, 8 (2), 1699-1707.

(143) Jäckel, F.; Perera, U. G. E.; Iancu, V.; Braun, K.-F.; Koch, N.; Rabe, J. P.; Hla, S.-W. Investigating Molecular Charge Transfer Complexes with a Low Temperature Scanning Tunneling Microscope. Phys. Rev. Lett. 2008, 100 (12), 126102.

(144) Braun, K.-F.; Hla, S. W. Charge Transfer in the TCNQ-Sexithiophene Complex. J. Chem. Phys. 2008, 129 (6), 64707.

(145) Sedona, F.; Di Marino, M.; Basagni, A.; Colazzo, L.; Sambi, M. Structurally Tunable Self-Assembled 2D Cocrystals of C60 and Porphyrins on the Ag (110) Surface. J. Phys. Chem. C 2014, 118 (3), 1587-1593.

(146) Mu, Z.; Shao, Q.; Ye, J.; Zeng, Z.; Zhao, Y.; Hng, H. H.; Boey, F. Y. C.; Wu, J.; Chen, $X$. Effect of Intermolecular Dipole-Dipole Interactions on Interfacial Supramolecular Structures of $C_{3}$-Symmetric Hexa- Peri -Hexabenzocoronene Derivatives ${ }^{\dagger}$. Langmuir 2011, 27 (4), 13141318.

(147) Fendt, L.-A.; Stöhr, M.; Wintjes, N.; Enache, M.; Jung, T. A.; Diederich, F. Modification of Supramolecular Binding Motifs Induced By Substrate Registry: Formation of Self-Assembled Macrocycles and Chain-Like Patterns. Chem. - Eur. J. 2009, 15 (42), 11139-11150.

(148) Won, S.-Y.; Kim, J.-H.; Kim, H.; Yoon, J. K.; Kahng, S.-J.; Kwon, Y.-K.; Park, Y. Linear and Hexagonal Porous Structures of an Organic Charge Acceptor Hexaaza-TriphenyleneHexacarbonitrile on $\mathrm{Au}(111)$ with $\mathrm{CN} \cdots \mathrm{CN}$ Dipolar Interactions. J. Phys. Chem. C 2013, 117 (41), 21371-21375.

(149) Wong, K.; Kwon, K.-Y.; Rao, B. V.; Liu, A.; Bartels, L. Effect of Halo Substitution on the Geometry of Arenethiol Films on Cu(111). J. Am. Chem. Soc. 2004, 126 (25), 7762-7763.

(150) The Molecule-Metal Interface; Koch, N., Ueno, N., Wee, A. T. S., Eds.; Wiley-VCH Verlag GmbH \& Co. KGaA: Weinheim, Germany, 2013.

(151) Flores, F.; Ortega, J.; Vázquez, H. Modelling Energy Level Alignment at Organic Interfaces and Density Functional Theory. Phys. Chem. Chem. Phys. 2009, 11 (39), 8658. 
(152) Ishii, H.; Sugiyama, K.; Ito, E.; Seki, K. Energy Level Alignment and Interfacial Electronic Structures at Organic/Metal and Organic/Organic Interfaces. Adv. Mater. 1999, 11 (8), $605-625$.

(153) Willenbockel, M.; Luftner, D.; Stadtmuller, B.; Koller, G.; Kumpf, C.; Soubatch, S.; Puschnig, P.; Ramsey, M. G.; Tautz, F. S. The Interplay between Interface Structure, Energy Level Alignment and Chemical Bonding Strength at Organic-Metal Interfaces. Phys. Chem. Chem. Phys. 2015, 17 (3), 1530-1548.

(154) Betti, M. G.; Kanjilal, A.; Mariani, C.; Vázquez, H.; Dappe, Y. J.; Ortega, J.; Flores, F. Barrier Formation at Organic Interfaces in a $\mathrm{Cu}(100)$-Benzenethiolate-Pentacene Heterostructure. Phys. Rev. Lett. 2008, 100 (2).

(155) Kolasinski, K. W. Surface Science: Foundations of Catalysis and Nanoscience, 3rd ed.; Wiley: Chichester, West Sussex ; Hoboken, N.J, 2012.

(156) Hammer, B.; Norskov, J. K. Why Gold Is the Noblest of All the Metals. Nature 1995, 376 (6537), 238-240.

(157) Hammer, B.; Nørskov, J. K. Theoretical Surface Science and Catalysis—calculations and Concepts. In Advances in Catalysis; Elsevier, 2000; Vol. 45, pp 71-129.

(158) Tkatchenko, A.; Romaner, L.; Hofmann, O. T.; Zojer, E.; Ambrosch-Draxl, C.; Scheffler, M. Van Der Waals Interactions Between Organic Adsorbates and at Organic/Inorganic Interfaces. MRS Bull. 2010, 35 (6), 435-442.

(159) Cohen, A. J.; Mori-Sánchez, P.; Yang, W. Challenges for Density Functional Theory. Chem Rev 2011, 112 (1), 289-320.

(160) Klimeš, J.; Michaelides, A. Perspective: Advances and Challenges in Treating van Der Waals Dispersion Forces in Density Functional Theory. J. Chem. Phys. 2012, 137 (12), 120901120901-120912.

(161) Liu, W.; Tkatchenko, A.; Scheffler, M. Modeling Adsorption and Reactions of Organic Molecules at Metal Surfaces. Acc. Chem. Res. 2014, 47 (11), 3369-3377.

(162) DiStasio, R. A. J.; Gobre, V. V.; Tkatchenko, A. Many-Body van Der Waals Interactions in Molecules and Condensed Matter. J. Phys. Condens. Matter 2014, 26 (21), 213202.

(163) Reilly, A. M.; Tkatchenko, A. Van Der Waals Dispersion Interactions in Molecular Materials: Beyond Pairwise Additivity. Chem. Sci. 2015, 6 (6), 3289-3301.

(164) Van Kampen, N. G.; Nijboer, B. R. A.; Schram, K. On the Macroscopic Theory of Van Der Waals Forces. Phys. Lett. A 1968, 26 (7), 307-308.

(165) Girard, C.; Bouju, X.; Martin, O. J. F.; Dereux, A.; Chavy, C.; Tang, H.; Joachim, C. Theoretical Atomic-Force-Microscopy Study of Adsorbed Fullerene Molecules. Phys. Rev. B 1993, 48 (20), 15417. 
(166) Girard, C.; Bouju, X. Coupled Electromagnetic Modes between a Corrugated Surface and a Thin Probe Tip. J. Chem. Phys. 1991, 95 (3), 2056-2064.

(167) Martínez, J. I.; Abad, E.; Beltrán, J. I.; Flores, F.; Ortega, J. Barrier Height Formation in Organic Blends/metal Interfaces: Case of Tetrathiafulvalene-tetracyanoquinodimethane $\mathrm{Au}(111)$. J. Chem. Phys. 2013, 139 (21), 214706.

(168) Fiedler, B.; Reckien, W.; Bredow, T.; Beck, J.; Sokolowski, M. Structure and Charge Transfer in Binary Ordered Monolayers of Two Sulfur-Containing Donor Molecules and TNAP on the Au(111) Surface. J. Phys. Chem. C 2014, 118 (6), 3035-3048.

(169) Umbach, T. R.; Fernandez-Torrente, I.; Ladenthin, J. N.; Pascual, J. I.; Franke, K. J. Enhanced Charge Transfer in a Monolayer of the Organic Charge Transfer Complex TTF-TNAP on $\mathrm{Au}(111)$. J. Phys. Condens. Matter 2012, 24 (35), 354003.

(170) Fernández-Torrente, I.; Kreikemeyer-Lorenzo, D.; Stróżecka, A.; Franke, K. J.; Pascual, J. I. Gating the Charge State of Single Molecules by Local Electric Fields. Phys. Rev. Lett. 2012, $108(3), 36801$.

(171) Stepanow, S.; Ohmann, R.; Leroy, F.; Lin, N.; Strunskus, T.; Wöll, C.; Kern, K. Rational Design of Two-Dimensional Nanoscale Networks by Electrostatic Interactions at Surfaces. $A C S$ Nano 2010, 4 (4), 1813-1820.

(172) Rodriguez, J. A.; Campbell, R. A.; Goodman, D. W. Electron Donor-electron Acceptor Interactions in Surface Metal-metal Bonds: The $\mathrm{Cu} / \operatorname{Re}(0001)$ and $\mathrm{Pd} / \operatorname{Re}(0001)$ Systems. J. Vac. Sci. Technol. A 1992, 10 (4), 2540-2545.

(173) Yu, M.; Xu, W.; Kalashnyk, N.; Benjalal, Y.; Nagarajan, S.; Masini, F.; Lægsgaard, E.; Hliwa, M.; Bouju, X.; Gourdon, A.; et al. From Zero to Two Dimensions: Supramolecular Nanostructures Formed from Perylene-3,4,9,10-Tetracarboxylic Diimide (PTCDI) and Ni on the $\mathrm{Au}(111)$ Surface through the Interplay between Hydrogen-Bonding and Electrostatic MetalOrganic Interactions. Nano Res. 2012, 5 (12), 903-916.

(174) Pyykkö, P. Understanding the Eighteen-Electron Rule. J. Organomet. Chem. 2006, 691 (21), 4336-4340.

(175) Pawin, G.; Wong, K. L.; Kim, D.; Sun, D.; Bartels, L.; Hong, S.; Rahman, T. S.; Carp, R.; Marsella, M. A Surface Coordination Network Based on Substrate-Derived Metal Adatoms with Local Charge Excess. Angew. Chem. Int. Ed. 2008, 47 (44), 8442-8445.

(176) MacLeod, J. M.; Ben Chaouch, Z.; Perepichka, D. F.; Rosei, F. Two-Dimensional SelfAssembly of a Symmetry-Reduced Tricarboxylic Acid. Langmuir 2013, 29 (24), 7318-7324.

(177) Batzill, M. The Surface Science of Graphene: Metal Interfaces, CVD Synthesis, Nanoribbons, Chemical Modifications, and Defects. Surf. Sci. Rep. 2012, 67 (3-4), 83-115.

(178) Zhang, Z.; Huang, H.; Yang, X.; Zang, L. Tailoring Electronic Properties of Graphene by $\Pi-\pi$ Stacking with Aromatic Molecules. J Phys Chem Lett 2011, 2 (22), 2897-2905. 
(179) Karmel, H. J.; Chien, T.; Demers-Carpentier, V.; Garramone, J. J.; Hersam, M. C. SelfAssembled Two-Dimensional Heteromolecular Nanoporous Molecular Arrays on Epitaxial Graphene. J. Phys. Chem. Lett. 2014, 5 (2), 270-274.

(180) Han, P.; Weiss, P. S. Electronic Substrate-Mediated Interactions. Surf. Sci. Rep. 2012, 67 (2), 19-81.

(181) Crommie, M. F.; Lutz, C. P.; Eigler, D. M. Imaging Standing Waves in a TwoDimensional Electron Gas. Nature 1993, 363 (6429), 524-527.

(182) Merrick, M. Substrate-Mediated Interactions on Solid Surfaces: Theory, Experiment, and Consequences for Thin-Film Morphology. Prog. Surf. Sci. 2003, 72 (5-8), 117-134.

(183) Repp, J.; Moresco, F.; Meyer, G.; Rieder, K.-H.; Hyldgaard, P.; Persson, M. Substrate Mediated Long-Range Oscillatory Interaction between Adatoms: $\mathrm{Cu} / \mathrm{Cu}(111)$. Phys. Rev. Lett. 2000, 85 (14), 2981-2984.

(184) Crommie, M. F.; Lutz, C. P.; Eigler, D. M. Confinement of Electrons to Quantum Corralson a Metal Surface. Science 1993, 262 (5131), 218-220.

(185) Crommie, M. F.; Lutz, C. P.; Eigler, D. M.; Heller, E. J. Quantum Corrals. Phys. Nonlinear Phenom. 1995, 83 (1-3), 98-108.

(186) Brune, H.; Wintterlin, J.; Ertl, G.; Behm, R. J. Direct Imaging of Adsorption Sites and Local Electronic Bond Effects on a Metal Surface: C/Al(111). EPL Europhys. Lett. 1990, 13 (2), 123.

(187) Knorr, N.; Brune, H.; Epple, M.; Hirstein, A.; Schneider, M. A.; Kern, K. Long-Range Adsorbate Interactions Mediated by a Two-Dimensional Electron Gas. Phys. Rev. B 2002, 65 (11).

(188) Ziegler, M.; Kröger, J.; Berndt, R.; Filinov, A.; Bonitz, M. Scanning Tunneling Microscopy and Kinetic Monte Carlo Investigation of Cesium Superlattices on $\operatorname{Ag}(111)$. Phys. Rev. B 2008, 78 (24), 245427.

(189) Cao, R. X.; Zhang, X. P.; Miao, B. F.; Zhong, Z. F.; Sun, L.; You, B.; Hu, A.; Ding, H. F. Self-Organized Gd Atomic Superlattice on $\operatorname{Ag}(111)$ : Scanning Tunneling Microscopy and Kinetic Monte Carlo Simulations. Surf. Sci. 2013, 610, 65-69.

(190) Sykes, E. C. H.; Han, P.; Kandel, S. A.; Kelly, K. F.; McCarty, G. S.; Weiss, P. S. Substrate-Mediated Interactions and Intermolecular Forces between Molecules Adsorbed on Surfaces. Acc. Chem. Res. 2003, 36 (12), 945-953.

(191) Barth, J. V.; Zambelli, T.; Wintterlin, J.; Schuster, R.; Ertl, G. Direct Observation of Mobility and Interactions of Oxygen Molecules Chemisorbed on the $\operatorname{Ag}(110)$ Surface. Phys. Rev. B 1997, 55 (19), 12902-12905.

(192) Mehlhorn, M.; Simic-Milosevic, V.; Jaksch, S.; Scheier, P.; Morgenstern, K. The Influence of the Surface State onto the Distance Distribution of Single Molecules and Small Molecular Clusters. Surf. Sci. 2010, 604 (19-20), 1698-1704. 
(193) Kamna, M. M.; Stranick, S. J.; Weiss, P. S. Imaging Substrate-Mediated Interactions. Science 1996, 274 (5284), 118-119.

(194) Dougherty, D. B.; Maksymovych, P.; Lee, J.; Yates, J. T. Local Spectroscopy of ImagePotential-Derived States: From Single Molecules to Monolayers of Benzene on $\mathrm{Cu}(111)$. Phys. Rev. Lett. 2006, 97 (23), 236806.

(195) Sykes, E. C. H.; Mantooth, B. A.; Han, P.; Donhauser, Z. J.; Weiss, P. S. SubstrateMediated Intermolecular Interactions: A Quantitative Single Molecule Analysis. J. Am. Chem. Soc. 2005, 127 (19), 7255-7260.

(196) Rojas, G.; Simpson, S.; Chen, X.; Kunkel, D. A.; Nitz, J.; Xiao, J.; Dowben, P. A.; Zurek, E.; Enders, A. Surface State Engineering of Molecule-molecule Interactions. Phys Chem Chem Phys 2012, 14 (14), 4971-4976.

(197) Zhu, Y.; Wyrick, J.; Cohen, K. D.; Magnone, K. M.; Holzke, C.; Salib, D.; Ma, Q.; Sun, D.; Bartels, L. Acetylene on $\mathrm{Cu}(111)$ : Imaging a Molecular Surface Arrangement with a Constantly Rearranging Tip. J. Phys. Condens. Matter 2012, 24 (35), 354005.

(198) Yokoyama, T.; Takahashi, T.; Shinozaki, K.; Okamoto, M. Quantitative Analysis of Long-Range Interactions between Adsorbed Dipolar Molecules on $\mathrm{Cu}(111)$. Phys. Rev. Lett. 2007, 98 (20), 206102.

(199) Goiri, E.; García-Lastra, J. M.; Corso, M.; Adb El-Fattah, Z. M.; Ortega, J. E.; de Oteyza, D. G. Understanding Periodic Dislocations in 2D Supramolecular Crystals: The PFP/Ag(111) Interface. J. Phys. Chem. Lett. 2012, 3 (7), 848-852.

(200) Wang, Y.; Ge, X.; Manzano, C.; Kröger, J.; Berndt, R.; Hofer, W. A.; Tang, H.; Cerda, J. Supramolecular Patterns Controlled by Electron Interference and Direct Intermolecular Interactions. J. Am. Chem. Soc. 2009, 131 (30), 10400-10402.

(201) Pawin, G.; Wong, K. L.; Kwon, K.; Bartels, L. A Homomolecular Porous Network at a $\mathrm{Cu}$ (111) Surface. Science 2006, 313 (5789), 961-962.

(202) Franke, K.; Schulze, G.; Henningsen, N.; Fernández-Torrente, I.; Pascual, J.; Zarwell, S.; Rück-Braun, K.; Cobian, M.; Lorente, N. Reducing the Molecule-Substrate Coupling in C60Based Nanostructures by Molecular Interactions. Phys. Rev. Lett. 2008, 100 (3), 36807.

(203) de Oteyza, D. G.; Silanes, I.; Ruiz-Osés, M.; Barrena, E.; Doyle, B. P.; Arnau, A.; Dosch, H.; Wakayama, Y.; Ortega, J. E. Balancing Intermolecular and Molecule-Substrate Interactions in Supramolecular Assemblies. Adv. Funct. Mater. 2009, 19 (2), 259-264.

(204) Goiri, E.; Matena, M.; El-Sayed, A.; Lobo-Checa, J.; Borghetti, P.; Rogero, C.; Detlefs, B.; Duvernay, J.; Ortega, J. E.; de Oteyza, D. G. Self-Assembly of Bicomponent Molecular Monolayers: Adsorption Height Changes and Their Consequences. Phys. Rev. Lett. 2014, 112 (11), 117602.

(205) Anderson, A. E.; Grillo, F.; Larrea, C. R.; Seljamäe-Green, R. T.; Früchtl, H. A.; Baddeley, C. J. Metallosupramolecular Assembly of $\mathrm{Cr}$ and P-Terphenyldinitrile by Dissociation of Metal Carbonyls on Au(111). J. Phys. Chem. C 2016, 120 (2), 1049-1055. 
(206) Tait, S. L.; Wang, Y.; Costantini, G.; Lin, N.; Baraldi, A.; Esch, F.; Petaccia, L.; Lizzit, S.; Kern, K. Metal-Organic Coordination Interactions in Fe-Terephthalic Acid Networks on $\mathrm{Cu}(100)$. J. Am. Chem. Soc. 2008, 130 (6), 2108-2113.

(207) Lingenfelder, M. A.; Spillmann, H.; Dmitriev, A.; Stepanow, S.; Lin, N.; Barth, J. V.; Kern, K. Towards Surface-Supported Supramolecular Architectures: Tailored Coordination Assembly of 1,4-Benzenedicarboxylate and Fe on $\mathrm{Cu}(100)$. Chem. - Eur. J. 2004, 10 (8), 1913 1919.

(208) Stepanow, S.; Lin, N.; Payer, D.; Schlickum, U.; Klappenberger, F.; Zoppellaro, G.; Ruben, M.; Brune, H.; Barth, J. V.; Kern, K. Surface-Assisted Assembly of 2D Metal-Organic Networks That Exhibit Unusual Threefold Coordination Symmetry. Angew. Chem. Int. Ed. 2007, $46(5), 710-713$.

(209) Classen, T.; Fratesi, G.; Costantini, G.; Fabris, S.; Stadler, F. L.; Kim, C.; de Gironcoli, S.; Baroni, S.; Kern, K. Templated Growth of Metal-Organic Coordination Chains at Surfaces. Angew. Chem. Int. Ed. 2005, 44 (38), 6142-6145.

(210) Seitsonen, A. P.; Lingenfelder, M.; Spillmann, H.; Dmitriev, A.; Stepanow, S.; Lin, N.; Kern, K.; Barth, J. V. Density Functional Theory Analysis of Carboxylate-Bridged Diiron Units in Two-Dimensional Metal-Organic Grids. J. Am. Chem. Soc. 2006, 128 (17), 5634-5635.

(211) Henningsen, N.; Rurali, R.; Limbach, C.; Drost, R.; Pascual, J. I.; Franke, K. J. SiteDependent Coordination Bonding in Self-Assembled Metal-Organic Networks. J Phys Chem Lett 2010, 2 (2), 55-61.

(212) Schlickum, U.; Decker, R.; Klappenberger, F.; Zoppellaro, G.; Klyatskaya, S.; Ruben, M.; Silanes, I.; Arnau, A.; Kern, K.; Brune, H.; et al. Metal-Organic Honeycomb Nanomeshes with Tunable Cavity Size. Nano Lett. 2007, 7 (12), 3813-3817.

(213) Weber, P. B.; Hellwig, R.; Paintner, T.; Lattelais, M.; Paszkiewicz, M.; Casado Aguilar, P.; Deimel, P. S.; Guo, Y.; Zhang, Y.-Q.; Allegretti, F.; et al. Surface-Guided Formation of an Organocobalt Complex. Angew. Chem. Int. Ed. 2016, 55 (19), 5754-5759.

(214) Björk, J.; Matena, M.; Dyer, M. S.; Enache, M.; Lobo-Checa, J.; Gade, L. H.; Jung, T. A.; Stöhr, M.; Persson, M. STM Fingerprint of Molecule-adatom Interactions in a SelfAssembled Metal-organic Surface Coordination Network on $\mathrm{Cu}(111)$. Phys. Chem. Chem. Phys. 2010, 12 (31), 8815-8821.

(215) Matena, M.; Stöhr, M.; Riehm, T.; Björk, J.; Martens, S.; Dyer, M. S.; Persson, M.; Lobo-Checa, J.; Müller, K.; Enache, M.; et al. Aggregation and Contingent Metal/Surface Reactivity of 1,3,8,10-Tetraazaperopyrene (TAPP) on $\mathrm{Cu}(111)$. Chem. - Eur. J. 2010, 16 (7), 2079-2091.

(216) Walch, H.; Dienstmaier, J.; Eder, G.; Gutzler, R.; Schlögl, S.; Sirtl, T.; Das, K.; Schmittel, M.; Lackinger, M. Extended Two-Dimensional Metal-Organic Frameworks Based on Thiolate-Copper Coordination Bonds. J Am Chem Soc 2011, 133 (20), 7909-7915. 
(217) Zhang, Y.-F.; Zhu, N.; Komeda, T. Mn-Coordinated Stillbenedicarboxylic Ligand Supramolecule Regulated by the Herringbone Reconstruction of Au(111). J. Phys. Chem. C 2007, 111 (45), 16946-16950.

(218) Meyer, J.; Nickel, A.; Ohmann, R.; Lokamani; Toher, C.; Ryndyk, D. A.; Garmshausen, Y.; Hecht, S.; Moresco, F.; Cuniberti, G. Tuning the Formation of Discrete Coordination Nanostructures. Chem. Commun. 2015, 51 (63), 12621-12624.

(219) Xu, W.; Wang, J.; Yu, M.; Lægsgaard, E.; Stensgaard, I.; Linderoth, T. R.; Hammer, B.; Wang, C.; Besenbacher, F. Guanine- and Potassium-Based Two-Dimensional Coordination Network Self-Assembled on Au(111). J. Am. Chem. Soc. 2010, 132 (45), 15927-15929.

(220) Hieulle, J.; Peyrot, D.; Jiang, Z.; Silly, F. Engineering Two-Dimensional Hybrid NaClOrganic Coordinated Nanoarchitectures on Metal Surfaces. Chem. Commun. 2015, 51 (67), $13162-13165$.

(221) Zhang, C.; Wang, L.; Xie, L.; Kong, H.; Tan, Q.; Cai, L.; Sun, Q.; Xu, W. Solventless Formation of G-Quartet Complexes Based on Alkali and Alkaline Earth Salts on Au(111). ChemPhysChem 2015, 16 (10), 2099-2105.

(222) Ahmadi, G.; Franke, K. J. Self-Assembly of Tetracyanonaphtho-Quinodimethane (TNAP) Based Metal-organic Networks on $\mathrm{Pb}(111)$ : Structural, Electronic, and Magnetic Properties. Appl. Surf. Sci. 2016, 373, 2-7.

(223) Saywell, A.; Greń, W.; Franc, G.; Gourdon, A.; Bouju, X.; Grill, L. Manipulating the Conformation of Single Organometallic Chains on Au(111). J. Phys. Chem. C 2014, 118 (3), 1719-1728.

(224) Zangwill, A. Modern Electrodynamics, Cambridge University Press.; Cambridge, 2013.

(225) Golze, D.; Iannuzzi, M.; Nguyen, M.-T.; Passerone, D.; Hutter, J. Simulation of Adsorption Processes at Metallic Interfaces: An Image Charge Augmented QM/MM Approach. J. Chem. Theory Comput. 2013, 9 (11), 5086-5097.

(226) Pronschinske, A.; Dougherty, D. B. Impact of Local Molecular Environment on the Decay of Image Potential States. J. Phys. Chem. Lett. 2010, 1 (17), 2613-2617.

(227) Suto, K.; Yoshimoto, S.; Itaya, K. Two-Dimensional Self-Organization of Phthalocyanine and Porphyrin: Dependence on the Crystallographic Orientation of Au. J. Am. Chem. Soc. 2003, 125 (49), 14976-14977.

(228) Yoshimoto, S.; Higa, N.; Itaya, K. Two-Dimensional Supramolecular Organization of Copper Octaethylporphyrin and Cobalt Phthalocyanine on Au(111): Molecular Assembly Control at an Electrochemical Interface. J. Am. Chem. Soc. 2004, 126 (27), 8540-8545.

(229) Zou, Y.; Kilian, L.; Schöll, A.; Schmidt, T.; Fink, R.; Umbach, E. Chemical Bonding of PTCDA on Ag Surfaces and the Formation of Interface States. Surf. Sci. 2006, 600 (6), 12401251. 
(230) Yoshimoto, S.; Honda, Y.; Ito, O.; Itaya, K. Supramolecular Pattern of Fullerene on 2D Bimolecular "Chessboard" Consisting of Bottom-up Assembly of Porphyrin and Phthalocyanine Molecules. J. Am. Chem. Soc. 2008, 130 (3), 1085-1092.

(231) Kröger, I.; Stadtmüller, B.; Kleimann, C.; Rajput, P.; Kumpf, C. Normal-Incidence XRay Standing-Wave Study of Copper Phthalocyanine Submonolayers on $\mathrm{Cu}(111)$ and $\mathrm{Au}(111)$. Phys. Rev. B 2011, 83 (19), 195414.

(232) Kröger, I.; Stadtmüller, B.; Stadler, C.; Ziroff, J.; Kochler, M.; Stahl, A.; Pollinger, F.; Lee, T.-L.; Zegenhagen, J.; Reinert, F.; et al. Submonolayer Growth of Copper-Phthalocyanine on $\operatorname{Ag}(111)$. New J. Phys. 2010, 12 (8), 83038.

(233) Tautz, F. S. Structure and Bonding of Large Aromatic Molecules on Noble Metal Surfaces: The Example of PTCDA. Prog. Surf. Sci. 2007, 82 (9-12), 479-520.

(234) Bürker, C.; Ferri, N.; Tkatchenko, A.; Gerlach, A.; Niederhausen, J.; Hosokai, T.; Duhm, S.; Zegenhagen, J.; Koch, N.; Schreiber, F. Exploring the Bonding of Large Hydrocarbons on Noble Metals: Diindoperylene on $\mathrm{Cu}(111), \mathrm{Ag}(111)$, and $\mathrm{Au}(111)$. Phys. Rev. B 2013, 87 (16), 165443.

(235) Duhm, S.; Gerlach, A.; Salzmann, I.; Bröker, B.; Johnson, R. L.; Schreiber, F.; Koch, N. PTCDA on $\mathrm{Au}(111), \mathrm{Ag}(111)$ and $\mathrm{Cu}(111)$ : Correlation of Interface Charge Transfer to Bonding Distance. Org. Electron. 2008, 9 (1), 111-118.

(236) de Oteyza, D. G.; El-Sayed, A.; Garcia-Lastra, J. M.; Goiri, E.; Krauss, T. N.; Turak, A.; Barrena, E.; Dosch, H.; Zegenhagen, J.; Rubio, A.; et al. Copper-Phthalocyanine Based Metalorganic Interfaces: The Effect of Fluorination, the Substrate, and Its Symmetry. J. Chem. Phys. 2010, 133 (21), 214703.

(237) Huang, H.; Chen, W.; Wee, A. T. S. Low-Temperature Scanning Tunneling Microscopy Investigation of Epitaxial Growth of F16CuPc Thin Films on $\operatorname{Ag}(111)$. J. Phys. Chem. C 2008, 112 (38), 14913-14918.

(238) Chen, L.; Li, H.; Wee, A. T. S. Nonlocal Chemical Reactivity at Organic-Metal Interfaces. ACS Nano 2009, 3 (11), 3684-3690.

(239) Heimel, G.; Duhm, S.; Salzmann, I.; Gerlach, A.; Strozecka, A.; Niederhausen, J.; Bürker, C.; Hosokai, T.; Fernandez-Torrente, I.; Schulze, G.; et al. Charged and Metallic Molecular Monolayers through Surface-Induced Aromatic Stabilization. Nat. Chem. 2013, 5 (3), $187-194$.

(240) Müller, K.; Moreno-López, J. C.; Gottardi, S.; Meinhardt, U.; Yildirim, H.; Kara, A.; Kivala, M.; Stöhr, M. Cyano-Functionalized Triarylamines on Coinage Metal Surfaces: Interplay of Intermolecular and Molecule-Substrate Interactions. Chem. - Eur. J. 2016, 22 (2), 581-589.

(241) Stadtmüller, B.; Sueyoshi, T.; Kichin, G.; Kröger, I.; Soubatch, S.; Temirov, R.; Tautz, F. S.; Kumpf, C. Commensurate Registry and Chemisorption at a Hetero-Organic Interface. Phys. Rev. Lett. 2012, 108 (10), 106103. 
(242) Cottin, M. C.; Schaffert, J.; Sonntag, A.; Karacuban, H.; Möller, R.; Bobisch, C. A. Supramolecular Architecture of Organic Molecules: PTCDA and CuPc on a $\mathrm{Cu}\left(\begin{array}{lll}1 & 1 & 1\end{array}\right)$ Substrate. Appl. Surf. Sci. 2012, 258 (6), 2196-2200.

(243) Wyrick, J.; Einstein, T. L.; Bartels, L. Chemical Insight from Density Functional Modeling of Molecular Adsorption: Tracking the Bonding and Diffusion of Anthracene Derivatives on $\mathrm{Cu}(111)$ with Molecular Orbitals. J. Chem. Phys. 2015, 142 (10), 101907.

(244) Guo, Q.; Li, F. Self-Assembled Alkanethiol Monolayers on Gold Surfaces: Resolving the Complex Structure at the Interface by STM. Phys. Chem. Chem. Phys. 2014, 16 (36), 1907419090.

(245) Rosei, F.; Schunack, M.; Naitoh, Y.; Jiang, P.; Gourdon, A.; Laegsgaard, E.; Stensgaard, I.; Joachim, C.; Besenbacher, F. Properties of Large Organic Molecules on Metal Surfaces. Prog. Surf. Sci. 2003, 71, 95-146.

(246) Schunack, M.; Linderoth, T. R.; Rosei, F.; Lægsgaard, E.; Stensgaard, I.; Besenbacher, F. Long Jumps in the Surface Diffusion of Large Molecules. Phys. Rev. Lett. 2002, 88 (15), 156102.

(247) Moresco, F.; Meyer, G.; Rieder, K. H.; Tang, H.; Gourdon, A.; Joachim, C. Conformational Changes of Single Molecules Induced by Scanning Tunneling Microscopy Manipulation: A Route to Molecular Switching. Phys. Rev. Lett. 2001, 86, 672-675.

(248) Shin, H.; Schwarze, A.; Diehl, R. D.; Pussi, K.; Colombier, A.; Gaudry, é.; Ledieu, J.; McGuirk, G. M.; Serkovic Loli, L. N.; Fournée, V.; et al. Structure and Dynamics of C 60 Molecules on Au(111). Phys. Rev. B 2014, 89 (24), 245428.

(249) Weckesser, J.; Barth, J. V.; Kern, K. Mobility and Bonding Transition of C 60 on Pd(110). Phys. Rev. B 2001, 64 (16), 161430(R).

(250) Weckesser, J.; Barth, J. V.; Kern, K. Direct Observation of Surface Diffusion of Large Organic Molecules at Metal Surfaces: PVBA on Pd(110). J. Chem. Phys. 1999, 110 (11), 5351.

(251) Otero, R.; Hummelink, F.; Sato, F.; Legoas, S. B.; Thostrup, P.; Laegsgaard, E.; Stensgaard, I.; Galvao, D. S.; Besenbacher, F. Lock-and-Key Effect in the Surface Diffusion of Large Organic Molecules Probed by STM. Nat Mater 2004, 3 (11), 779-782.

(252) Haq, S.; Wit, B.; Sang, H.; Floris, A.; Wang, Y.; Wang, J.; Pérez-García, L.; Kantorovitch, L.; Amabilino, D. B.; Raval, R. A Small Molecule Walks Along a Surface Between Porphyrin Fences That Are Assembled In Situ. Angew. Chem. Int. Ed. 2015, 54 (24), 7101-7105.

(253) Rossel, F.; Brodard, P.; Patthey, F.; Richardson, N. V.; Schneider, W.-D. Modified Herringbone Reconstruction on $\mathrm{Au}(111)$ Induced by Self-Assembled Azure A Islands. Surf. Sci. 2008, 602 (14), L115-L117.

(254) Jewell, A. D.; Tierney, H. L.; Sykes, E. C. H. Gently Lifting Gold's Herringbone Reconstruction: Trimethylphosphine on Au(111). Phys. Rev. B 2010, 82 (20), 205401. 
(255) Lo, Y.-Y.; Chang, J.-H.; Hoffmann, G.; Su, W.-B.; Wu, C.-I.; Chang, C.-S. A Comparative Study on the Adsorption Behavior of Pentacene and Perfluoropentacene Molecules on Au(111) Surfaces. Jpn. J. Appl. Phys. 2013, 52, 101601.

(256) Schunack, M.; Petersen, L.; Kühnle, A.; Lægsgaard, E.; Stensgaard, I.; Johannsen, I.; Besenbacher, F. Anchoring of Organic Molecules to a Metal Surface: HtBDC on Cu(110). Phys. Rev. Lett. 2001, 86 (3), 456-459.

(257) Rosei, F.; Schunack, M.; Jiang, P.; Gourdon, A.; Lægsgaard, E.; Stensgaard, I.; Joachim, C.; Besenbacher, F. Organic Molecules Acting as Templates on Metal Surfaces. Science 2002, 296 (5566), 328-331.

(258) Aakeröy, C. B.; Seddon, K. R. The Hydrogen Bond and Crystal Engineering. Chem. Soc. Rev. 1993, 22 (6), 397-407.

(259) Slater, A. G.; Perdigão, L. M. A.; Beton, P. H.; Champness, N. R. Surface-Based Supramolecular Chemistry Using Hydrogen Bonds. Acc. Chem. Res. 2014, 47 (12), 3417-3427.

(260) Perdigão, L. M. A.; Perkins, E. W.; Ma, J.; Staniec, P. A.; Rogers, B. L.; Champness, N. R.; Beton, P. H. Bimolecular Networks and Supramolecular Traps on Au(111). J. Phys. Chem. B 2006, 110 (25), 12539-12542.

(261) Silly, F.; Shaw, A. Q.; Castell, M. R.; Briggs, G. A. D.; Mura, M.; Martsinovich, N.; Kantorovich, L. Melamine Structures on the Au(111) Surface. J. Phys. Chem. C 2008, 112 (30), $11476-11480$.

(262) Zhang, H.-M.; Xie, Z.-X.; Long, L.-S.; Zhong, H.-P.; Zhao, W.; Mao, B.-W.; Xu, X.; Zheng, L.-S. One-Step Preparation of Large-Scale Self-Assembled Monolayers of Cyanuric Acid and Melamine Supramolecular Species on Au(111) Surfaces. J. Phys. Chem. C 2008, 112 (11), 4209-4218.

(263) Staniec, P. A.; Perdigao, L. M. A.; Rogers, B. L.; Champness, N. R.; Beton, P. H. Honeycomb Networks and Chiral Superstructures Formed by Cyanuric Acid and Melamine on Au (111). J. Phys. Chem. C 2007, 111 (2), 886-893.

(264) Perdigão, L.; Fontes, G.; Rogers, B.; Oxtoby, N.; Goretzki, G.; Champness, N.; Beton, P. Coadsorbed NTCDI-Melamine Mixed Phases on Ag-Si(111). Phys. Rev. B 2007, 76 (24), 245402.

(265) Schmitz, C. H.; Ikonomov, J.; Sokolowski, M. Two Commensurate Hydrogen-Bonded Monolayer Structures of Melamine on Ag(111). Surf. Sci. 2011, 605 (1-2), 1-6.

(266) Mura, M.; Martsinovich, N.; Kantorovich, L. Theoretical Study of Melamine Superstructures and Their Interaction with the Au(111) Surface. Nanotechnology 2008, 19 (46), 465704.

(267) Mura, M.; Silly, F.; Burlakov, V.; Castell, M. R.; Briggs, G. A. D.; Kantorovich, L. N. Formation Mechanism for a Hybrid Supramolecular Network Involving Cooperative Interactions. Phys. Rev. Lett. 2012, 108 (17), 176103. 
(268) Ranganathan, A.; Pedireddi, V. R.; Rao, C. N. R. Hydrothermal Synthesis of Organic Channel Structures: 1:1 Hydrogen-Bonded Adducts of Melamine with Cyanuric and Trithiocyanuric Acids. J. Am. Chem. Soc. 1999, 121 (8), 1752-1753.

(269) Whitesides, G. M.; Mathias, J. P.; Seto, C. T. Molecular Self-Assembly and Nanochemistry: A Chemical Strategy for the Synthesis of Nanostructures. Science 1991, 254 (5036), 1312-1319.

(270) Whitesides, G. M.; Simanek, E. E.; Mathias, J. P.; Seto, C. T.; Chin, D.; Mammen, M.; Gordon, D. M. Noncovalent Synthesis: Using Physical-Organic Chemistry To Make Aggregates. Acc. Chem. Res. 1995, 28 (1), 37-44.

(271) Perdigão, L. M. A.; Champness, N. R.; Beton, P. H. Surface Self-Assembly of the Cyanuric Acid-melamine Hydrogen Bonded Network. Chem. Commun. 2006, No. 5, 538-540.

(272) Xu, W.; Dong, M.; Gersen, H.; Rauls, E.; Vázquez-Campos, S.; Crego-Calama, M.; Reinhoudt, D. N.; Stensgaard, I.; Laegsgaard, E.; Linderoth, T. R.; et al. Cyanuric Acid and Melamine on $\mathrm{Au}(111)$ : Structure and Energetics of Hydrogen-Bonded Networks. Small 2007, 3 (5), 854-858.

(273) Bombis, C.; Kalashnyk, N.; Xu, W.; Laegsgaard, E.; Besenbacher, F.; Linderoth, T. R. Hydrogen-Bonded Molecular Networks of Melamine and Cyanuric Acid on Thin Films of $\mathrm{NaCl}$ on $\mathrm{Au}(111)$. Small 2009, 5 (19), 2177-2182.

(274) Madueno, R.; Räisänen, M. T.; Silien, C.; Buck, M. Functionalizing Hydrogen-Bonded Surface Networks with Self-Assembled Monolayers. Nature 2008, 454 (7204), 618-621.

(275) Lombana, A.; Rinfray, C.; Volatron, F.; Izzet, G.; Battaglini, N.; Alves, S.; Decorse, P.; Lang, P.; Proust, A. Surface Organization of Polyoxometalate Hybrids Steered by a 2D Supramolecular PTCDI/Melamine Network. J. Phys. Chem. C 2016, 120 (5), 2837-2845.

(276) Topple, J.; Burke, S.; Fostner, S.; Grütter, P. Thin Film Evolution: Dewetting Dynamics of a Bimodal Molecular System. Phys. Rev. B 2009, 79 (20), 205414.

(277) Cañas-Ventura, M. E.; Xiao, W.; Wasserfallen, D.; Müllen, K.; Brune, H.; Barth, J. V.; Fasel, R. Self-Assembly of Periodic Bicomponent Wires and Ribbons. Angew. Chem. Int. Ed. 2007, 46 (11), 1814-1818.

(278) Silly, F.; Shaw, A. Q.; Castell, M. R.; Briggs, G. A. D. A Chiral Pinwheel Supramolecular Network Driven by the Assembly of PTCDI and Melamine. Chem. Commun. 2008, No. 16, 1907-1909.

(279) Mura, M.; Silly, F.; Briggs, G. A. D.; Castell, M. R.; Kantorovich, L. N. H-Bonding Supramolecular Assemblies of PTCDI Molecules on the Au(111) Surface. J. Phys. Chem. C 2009, 113 (52), 21840-21848.

(280) Keeling, D. L.; Oxtoby, N. S.; Wilson, C.; Humphry, M. J.; Champness, N. R.; Beton, P. H. Assembly and Processing of Hydrogen Bond Induced Supramolecular Nanostructures. Nano Lett. 2003, 3 (1), 9-12. 
(281) Korolkov, V. V.; Svatek, S. A.; Allen, S.; Roberts, C. J.; Tendler, S. J. B.; Taniguchi, T.; Watanabe, K.; Champness, N. R.; Beton, P. H. Bimolecular Porous Supramolecular Networks Deposited from Solution on Layered Materials: Graphite, Boron Nitride and Molybdenum Disulphide. Chem. Commun. 2014, 50 (64), 8882-8885.

(282) Theobald, J. A.; Oxtoby, N. S.; Phillips, M. A.; Champness, N. R.; Beton, P. H. Controlling Molecular Deposition and Layer Structure with Supramolecular Surface Assemblies. Nature 2003, 424 (6952), 1029-1031.

(283) Staniec, P. A.; Perdigão, L. M. A.; Saywell, A.; Champness, N. R.; Beton, P. H. Hierarchical Organisation on a Two-Dimensional Supramolecular Network. ChemPhysChem 2007, 8 (15), 2177-2181.

(284) Silly, F.; Shaw, A. Q.; Briggs, G. A. D.; Castell, M. R. Epitaxial Ordering of a Perylenetetracarboxylic Diimide-Melamine Supramolecular Network Driven by the Au(111)(22×3) Reconstruction. Appl. Phys. Lett. 2008, 92 (2), 23102.

(285) Sassi, M.; Oison, V.; Debierre, J.-M. First Principle Study of a Bimolecular Thin Film on $\operatorname{Ag}\left(\begin{array}{lll}1 & 1 & 1)\end{array}\right)$ Surface. Surf. Sci. 2008, 602 (17), 2856-2862.

(286) Perdigão, L. M. A.; Saywell, A.; Fontes, G. N.; Staniec, P. A.; Goretzki, G.; Phillips, A. G.; Champness, N. R.; Beton, P. H. Functionalized Supramolecular Nanoporous Arrays for Surface Templating. Chem. - Eur. J. 2008, 14 (25), 7600-7607.

(287) Gerlach, A.; Sellner, S.; Kowarik, S.; Schreiber, F. In-Situ X-Ray Scattering Studies of OFET Interfaces. Phys. Status Solidi A 2008, 205 (3), 461-474.

(288) Schmitz-Hübsch, T.; Fritz, T.; Staub, R.; Back, A.; Armstrong, N. R.; Leo, K. Structure of 3,4,9,10-Perylene-Tetracarboxylic-Dianhydride Grown on Reconstructed and Unreconstructed Au(100). Surf. Sci. 1999, 437 (1-2), 163-172.

(289) Glöckler, K.; Seidel, C.; Soukopp, A.; Sokolowski, M.; Umbach, E.; Böhringer, M.; Berndt, R.; Schneider, W.-D. Highly Ordered Structures and Submolecular Scanning Tunnelling Microscopy Contrast of PTCDA and DM-PBDCI Monolayers on Ag(111) and Ag(110). Surf. Sci. 1998, 405 (1), 1-20.

(290) Umbach, E.; Glöckler, K.; Sokolowski, M. Surface "architecture" with Large Organic Molecules: Interface Order and Epitaxy. Surf. Sci. 1998, 402-404, 20-31.

(291) Hauschild, A.; Karki, K.; Cowie, B.; Rohlfing, M.; Tautz, F.; Sokolowski, M. Molecular Distortions and Chemical Bonding of a Large $\pi$-Conjugated Molecule on a Metal Surface. Phys. Rev. Lett. 2005, 94 (3), 36106.

(292) Swarbrick, J. C.; Rogers, B. L.; Champness, N. R.; Beton, P. H. Hydrogen-Bonded PTCDA-Melamine Networks and Mixed Phases. J. Phys. Chem. B 2006, 110, 6110-6114.

(293) Swarbrick, J. C.; Ma, J.; Theobald, J. A.; Oxtoby, N. S.; O’Shea, J. N.; Champness, N. R.; Beton, P. H. Square, Hexagonal, and Row Phases of PTCDA and PTCDI on Ag-Si(111) $\times$ $\mathrm{R}$ 30. J. Phys. Chem. B 2005, 109, 12167-12174. 
(294) Silly, F.; Weber, U. K.; Shaw, A. Q.; Burlakov, V. M.; Castell, M. R.; Briggs, G. A. D.; Pettifor, D. G. Deriving Molecular Bonding from a Macromolecular Self-Assembly Using Kinetic Monte Carlo Simulations. Phys. Rev. B 2008, 77 (20), 201408.

(295) Sun, X.; Jonkman, H. T.; Silly, F. Tailoring Two-Dimensional PTCDA-melamine SelfAssembled Architectures at Room Temperature by Tuning Molecular Ratio. Nanotechnology 2010, 21, 165602.

(296) Ruiz-Osés, M.; González-Lakunza, N.; Silanes, I.; Gourdon, A.; Arnau, A.; Ortega, J. E. Self-Assembly of Heterogeneous Supramolecular Structures with Uniaxial Anisotropy. J. Phys. Chem. B 2006, 110 (51), 25573-25577.

(297) Ruiz-Osés, M.; González-Lakunza, N.; Silanes, I.; Gourdon, A.; Arnau, A.; Ortega, J. E. Self-Assembly of Heterogeneous Supramolecular Structures with Uniaxial Anisotropy. J Phys Chem B 2006, 110 (51), 25573-25577.

(298) Ruiz-Osés, M.; Kampen, T.; González-Lakunza, N.; Silanes, I.; Schmidt-Weber, P. M.; Gourdon, A.; Arnau, A.; Horn, K.; Ortega, J. E. Spectroscopic Fingerprints of Amine and Imide Functional Groups in Self-Assembled Monolayers. ChemPhysChem 2007, 8 (11), 1722-1726.

(299) Ruiz-Osés, M.; de Oteyza, D. G.; Fernández-Torrente, I.; Gonzalez-Lakunza, N.; Schmidt-Weber, P. M.; Kampen, T.; Horn, K.; Gourdon, A.; Arnau, A.; Ortega, J. E. NonCovalent Interactions in Supramolecular Assemblies Investigated with Electron Spectroscopies. ChemPhysChem 2009, 10 (6), 896-900.

(300) Gonzalez-Lakunza, N.; Cañas-Ventura, M. E.; Ruffieux, P.; Rieger, R.; Müllen, K.; Fasel, R.; Arnau, A. Hydrogen-Bonding Fingerprints in Electronic States of Two-Dimensional Supramolecular Assemblies. ChemPhysChem 2009, 10 (17), 2943-2946.

(301) Cañas-Ventura, M. E.; Aït-Mansour, K.; Ruffieux, P.; Rieger, R.; Müllen, K.; Brune, H.; Fasel, R. Complex Interplay and Hierarchy of Interactions in Two-Dimensional Supramolecular Assemblies. ACS Nano 2011, 5 (1), 457-469.

(302) Cañas-Ventura, M. E.; Xiao, W.; Ruffieux, P.; Rieger, R.; MÃ¹/4len, K.; Brune, H.; Fasel, R. Stabilization of Bimolecular Islands on Ultrathin $\mathrm{NaCl}$ Films by a Vicinal Substrate. Surf. Sci. 2009, 603 (15), 2294-2299.

(303) Aït-Mansour, K.; Cañas-Ventura, M. E.; Ruffieux, P.; Jaafar, R.; Bieri, M.; Rieger, R.; Müllen, K.; Fasel, R.; Gröning, O. Strain-Relief Pattern as Guide for the Formation of SurfaceSupported Bimolecular Nanoribbons. Appl. Phys. Lett. 2009, 95, 143111.

(304) Kalashnyk, N.; Yu, M.; Barattin, R.; Benjalal, Y.; Hliwa, M.; Joachim, C.; Laegsgaard, E.; Besenbacher, F.; Gourdon, A.; Bouju, X.; et al. Bicomponent Hydrogen-Bonded Nanostructures Formed by Two Complementary Molecular Landers on Au(111). Chem. Commun. 2014, 50 (73), 10619-10621.

(305) Yu, M.; Kalashnyk, N.; Xu, W.; Barattin, R.; Benjalal, Y.; Lægsgaard, E.; Stensgaard, I.; Hliwa, M.; Bouju, X.; Gourdon, A.; et al. Supramolecular Architectures on Surfaces Formed through Hydrogen Bonding Optimized in Three Dimensions. ACS Nano 2010, 4 (7), 4097-4109. 
(306) Ma, J.; Rogers, B. L.; Humphry, M. J.; Ring, D. J.; Goretzki, G.; Champness, N. R.; Beton, P. H. Dianhydride-Amine Hydrogen Bonded Perylene Tetracarboxylic Dianhydride and Tetraaminobenzene Rows. J. Phys. Chem. B 2006, 110 (25), 12207-12210.

(307) Nguyen, M.-T.; Pignedoli, C. A.; Treier, M.; Fasel, R.; Passerone, D. The Role of van Der Waals Interactions in Surface-Supported Supramolecular Networks. Phys. Chem. Chem. Phys. 2010, 12 (4), 992.

(308) Treier, M.; Nguyen, M.-T.; Richardson, N. V.; Pignedoli, C.; Passerone, D.; Fasel, R. Tailoring Low-Dimensional Organic Semiconductor Nanostructures. Nano Lett 2008, 9 (1), 126131.

(309) Sun, X.; Mura, M.; Jonkman, H. T.; Kantorovich, L. N.; Silly, F. Fabrication of a Complex Two-Dimensional Adenine-Perylene-3,4,9,10-Tetracarboxylic Dianhydride Chiral Nanoarchitecture through Molecular Self-Assembly. J Phys Chem C 2011, 116 (3), 2493-2499.

(310) Liang, H.; Sun, W.; Jin, X.; Li, H.; Li, J.; Hu, X.; Teo, B. K.; Wu, K. Two-Dimensional Molecular Porous Networks Formed by Trimesic Acid and 4,4'-Bis(4-Pyridyl)biphenyl on $\mathrm{Au}(111)$ through Hierarchical Hydrogen Bonds: Structural Systematics and Control of Nanopore Size and Shape. Angew. Chem. Int. Ed. 2011, 50 (33), 7562-7566.

(311) Kunkel, D. A.; Hooper, J.; Bradley, B.; Schlueter, L.; Rasmussen, T.; Costa, P.; Beniwal, S.; Ducharme, S.; Zurek, E.; Enders, A. 2D Cocrystallization from H-Bonded Organic Ferroelectrics. J. Phys. Chem. Lett. 2016, 7 (3), 435-440.

(312) Kühnle, A.; Linderoth, T. R.; Besenbacher, F. Enantiospecific Adsorption of Cysteine at Chiral Kink Sites on Au(110)-(1×2). J. Am. Chem. Soc. 2006, 128 (4), 1076-1077.

(313) Kuhnle, A.; Linderoth, T. R.; Hammer, B.; Besenbacher, F. Chiral Recognition in Dimerization of Adsorbed Cysteine Observed by Scanning Tunnelling Microscopy. Nature 2002, 415 (6874), 891-893.

(314) Iwai, H.; Egawa, C. Molecular Orientation and Intermolecular Interaction in Alanine on $\mathrm{Cu}(001)$. Langmuir 2010, 26 (4), 2294-2300.

(315) Eralp, T.; Shavorskiy, A.; Zheleva, Z. V.; Held, G.; Kalashnyk, N.; Ning, Y.; Linderoth, T. R. Global and Local Expression of Chirality in Serine on the $\mathrm{Cu}\{110\}$ Surface. Langmuir 2010, 26 (24), 18841-18851.

(316) Otero, R.; Lukas, M.; Kelly, R. E. A.; Xu, W.; Laegsgaard, E.; Stensgaard, I.; Kantorovich, L. N.; Besenbacher, F. Elementary Structural Motifs in a Random Network of Cytosine Adsorbed on a Gold(111) Surface. Science 2008, 319 (5861), 312-315.

(317) Otero, R.; Schöck, M.; Molina, L. M.; Laegsgaard, E.; Stensgaard, I.; Hammer, B.; Besenbacher, F. Guanine Quartet Networks Stabilized by Cooperative Hydrogen Bonds. Angew. Chem. Int. Ed. 2005, 44 (15), 2270-2275.

(318) Xu, W.; Kelly, R. E. A.; Otero, R.; Schöck, M.; Lægsgaard, E.; Stensgaard, I.; Kantorovich, L. N.; Besenbacher, F. Probing the Hierarchy of Thymine-Thymine Interactions in 
Self-Assembled Structures by Manipulation with Scanning Tunneling Microscopy. Small 2007, 3 (12), 2011-2014.

(319) Otero, R.; Xu, W.; Lukas, M.; Kelly, R. E. A.; Laegsgaard, E.; Stensgaard, I.; Kjems, J.; Kantorovich, L. N.; Besenbacher, F. Specificity of Watson-Crick Base Pairing on a Solid Surface Studied at the Atomic Scale. Angew. Chem. Int. Ed. 2008, 47 (50), 9673-9676.

(320) Xu, W.; Wang, J.; Jacobsen, M. F.; Mura, M.; Yu, M.; Kelly, R. E. A.; Meng, Q.; Laegsgaard, E.; Stensgaard, I.; Linderoth, T. R.; et al. Supramolecular Porous Network Formed by Molecular Recognition between Chemically Modified Nucleobases Guanine and Cytosine. Angew. Chem. Int. Ed. 2010, 49 (49), 9373-9377.

(321) Wang, Y.; Lingenfelder, M.; Classen, T.; Costantini, G.; Kern, K. Ordering of Dipeptide Chains on $\mathrm{Cu}$ Surfaces through 2D Cocrystallization. J. Am. Chem. Soc. 2007, 129 (51), 1574215743.

(322) Urgel, J. I.; Vijayaraghavan, S.; Ecija, D.; Auwärter, W.; Barth, J. V. Tetracene Confinement in L-Methionine Gratings on the Ag(111) Surface. Surf. Sci. 2016, 643, 87-90.

(323) Tait, S. L.; Langner, A.; Lin, N.; Stepanow, S.; Rajadurai, C.; Ruben, M.; Kern, K. OneDimensional Self-Assembled Molecular Chains on $\mathrm{Cu}(100)$ : Interplay between Surface-Assisted Coordination Chemistry and Substrate Commensurability. J. Phys. Chem. C 2007, 111 (29), 10982-10987.

(324) Tait, S. L.; Langner, A.; Lin, N.; Chandrasekar, R.; Fuhr, O.; Ruben, M.; Kern, K. Assembling Isostructural Metal-Organic Coordination Architectures on $\mathrm{Cu}(100), \mathrm{Ag}(100)$ and Ag(111) Substrates. ChemPhysChem 2008, 9 (17), 2495-2499.

(325) Lin, N.; Langner, A.; Tait, S. L.; Rajadurai, C.; Ruben, M.; Kern, K. Template-Directed Supramolecular Self-Assembly of Coordination Dumbbells at Surfaces. Chem. Commun. 2007, No. 46, 4860-4862.

(326) Langner, A.; Tait, S. L.; Lin, N.; Chandrasekar, R.; Ruben, M.; Kern, K. Two- to OneDimensional Transition of Self-Assembled Coordination Networks at Surfaces by Organic Ligand Addition. Chem Commun 2009, No. 18, 2502-2504.

(327) Langner, A.; Tait, S. L.; Lin, N.; Rajadurai, C.; Ruben, M.; Kern, K. Self-Recognition and Self-Selection in Multicomponent Supramolecular Coordination Networks on Surfaces. Proc. Natl. Acad. Sci. 2007, 104 (46), 17927-17930.

(328) Langner, A.; Tait, S. L.; Lin, N.; Chandrasekar, R.; Ruben, M.; Kern, K. Ordering and Stabilization of Metal-Organic Coordination Chains by Hierarchical Assembly through Hydrogen Bonding at a Surface. Angew. Chem. Int. Ed. 2008, 47 (46), 8835-8838.

(329) Langner, A.; Tait, S. L.; Lin, N.; Chandrasekar, R.; Meded, V.; Fink, K.; Ruben, M.; Kern, K. Selective Coordination Bonding in Metallo-Supramolecular Systems on Surfaces. Angew. Chem. Int. Ed. 2012, 51 (18), 4327-4331. 
(330) Stepanow, S.; Lin, N.; Barth, J. V.; Kern, K. Surface-Template Assembly of TwoDimensional Metal-Organic Coordination Networks. J. Phys. Chem. B 2006, 110 (46), 2347223477.

(331) Shi, Z;; Lin, N. Structural and Chemical Control in Assembly of Multicomponent Metal-Organic Coordination Networks on a Surface. J. Am. Chem. Soc. 2010, 132 (31), 1075610761.

(332) Lin, T.; Shang, X. S.; Liu, P. N.; Lin, N. Multicomponent Assembly of Supramolecular Coordination Polygons on a Au(111) Surface. J. Phys. Chem. C 2013, 117 (44), 23027-23033.

(333) Van Siclen, C. D. Information Entropy of Complex Structures. Phys. Rev. E 1997, 56 (5), 5211-5215.

(334) Ecija, D.; Urgel, J. I.; Papageorgiou, A. C.; Joshi, S.; Auwarter, W.; Seitsonen, A. P.; Klyatskaya, S.; Ruben, M.; Fischer, S.; Vijayaraghavan, S.; et al. Five-Vertex Archimedean Surface Tessellation by Lanthanide-Directed Molecular Self-Assembly. Proc. Natl. Acad. Sci. 2013, 110 (17), 6678-6681.

(335) Urgel, J. I.; Ecija, D.; Auwärter, W.; Papageorgiou, A. C.; Seitsonen, A. P.; Vijayaraghavan, S.; Joshi, S.; Fischer, S.; Reichert, J.; Barth, J. V. Five-Vertex Lanthanide Coordination on Surfaces: A Route to Sophisticated Nanoarchitectures and Tessellations. $J$. Phys. Chem. C 2014, 118 (24), 12908-12915.

(336) Urgel, J. I.; Ecija, D.; Auwärter, W.; Stassen, D.; Bonifazi, D.; Barth, J. V. Orthogonal Insertion of Lanthanide and Transition-Metal Atoms in Metal-Organic Networks on Surfaces. Angew. Chem. Int. Ed. 2015, 54 (21), 6163-6167.

(337) Urgel, J. I.; Cirera, B.; Wang, Y.; Auwärter, W.; Otero, R.; Gallego, J. M.; Alcamí, M.; Klyatskaya, S.; Ruben, M.; Martín, F.; et al. Surface-Supported Robust 2D LanthanideCarboxylate Coordination Networks. Small 2015, 11 (47), 6358-6364.

(338) Lyu, G.; Zhang, Q.; Urgel, J. I.; Kuang, G.; Auwärter, W.; Ecija, D.; Barth, J. V.; Lin, N. Tunable Lanthanide-Directed Metallosupramolecular Networks by Exploiting Coordinative Flexibility through Ligand Stoichiometry. Chem Commun 2016, 52 (8), 1618-1621.

(339) Buchner, F.; Zillner, E.; Röckert, M.; Gläßel, S.; Steinrück, H.-P.; Marbach, H. SubstrateMediated Phase Separation of Two Porphyrin Derivatives on $\mathrm{Cu}(111)$. Chem. - Eur. J. 2011, 17 (37), 10226-10229.

(340) Wintjes, N.; Lobo-Checa, J.; Hornung, J.; Samuely, T.; Diederich, F.; Jung, T. A. TwoDimensional Phase Behavior of a Bimolecular Porphyrin System at the Solid-Vacuum Interface. J. Am. Chem. Soc. 2010, 132 (21), 7306-7311.

(341) Song, W.; Martsinovich, N.; Heckl, W. M.; Lackinger, M. Born-Haber Cycle for Monolayer Self-Assembly at the Liquid-Solid Interface: Assessing the Enthalpic Driving Force. J. Am. Chem. Soc. 2013, 135 (39), 14854-14862. 
(342) Koudia, M.; Abel, M.; Maurel, C.; Bliek, A.; Catalin, D.; Mossoyan, M.; Mossoyan, J.C.; Porte, L. Influence of Chlorine Substitution on the Self-Assembly of Zinc Phthalocyanine. $J$. Phys. Chem. B 2006, 110 (20), 10058-10062.

(343) Abel, M.; Oison, V.; Koudia, M.; Maurel, C.; Katan, C.; Porte, L. Designing a New TwoDimensional Molecular Layout by Hydrogen Bonding. ChemPhysChem 2006, 7, 82-85.

(344) Calmettes, B.; Nagarajan, S.; Gourdon, A.; Abel, M.; Porte, L.; Coratger, R. Bicomponent Supramolecular Packing in Flexible Phthalocyanine Networks. Angew. Chem. Int. Ed. 2008, 47 (37), 6994-6998.

(345) Calmettes, B.; Nagarajan, S.; Gourdon, A.; Benjalal, Y.; Bouju, X.; Abel, M.; Porte, L.; Coratger, R. Properties of Penta-Tert-Butylcorannulene Molecules Inserted in Phthalocyanine Networks Studied by Low-Temperature Scanning Tunneling Microscopy. J. Phys. Chem. C 2009, 113 (50), 21169-21176.

(346) de Oteyza, D. G.; Barrena, E.; Dosch, H.; Ortega, J. E.; Wakayama, Y. Tunable Symmetry and Periodicity in Binary Supramolecular Nanostructures. Phys. Chem. Chem. Phys. 2011, 13 (10), 4220-4223.

(347) Barrena, E.; de Oteyza, D. G.; Dosch, H.; Wakayama, Y. 2D Supramolecular SelfAssembly of Binary Organic Monolayers. ChemPhysChem 2007, 8 (13), 1915-1918.

(348) Krauss, T. N.; Barrena, E.; Dosch, H.; Wakayama, Y. Supramolecular Assembly of a 2D Binary Network of Pentacene and Phthalocyanine on $\mathrm{Cu}(100)$. ChemPhysChem 2009, 10 (14), 2445-2448.

(349) Wakayama, Y.; de Oteyza, D. G.; Garcia-Lastra, J. M.; Mowbray, D. J. Solid-State Reactions in Binary Molecular Assemblies of F16CuPc and Pentacene. ACS Nano 2011, 5 (1), 581-589.

(350) de Oteyza, D. G.; Garcia-Lastra, J. M.; Goiri, E.; El-Sayed, A.; Wakayama, Y.; Ortega, J. E. Asymmetric Response toward Molecular Fluorination in Binary CopperPhthalocyanine/Pentacene Assemblies. J. Phys. Chem. C 2014, 118 (32), 18626-18630.

(351) El-Sayed, A.; Mowbray, D. J.; García-Lastra, J. M.; Rogero, C.; Goiri, E.; Borghetti, P.; Turak, A.; Doyle, B. P.; Dell'Angela, M.; Floreano, L.; et al. Supramolecular EnvironmentDependent Electronic Properties of Metal-Organic Interfaces. J. Phys. Chem. C 2012, 116 (7), 4780-4785.

(352) Huang, Y. L.; Chen, W.; Li, H.; Ma, J.; Pflaum, J.; Wee, A. T. S. Tunable TwoDimensional Binary Molecular Networks. Small 2010, 6 (1), 70-75.

(353) Huang, Y. L.; Li, H.; Ma, J.; Huang, H.; Chen, W.; Wee, A. T. S. Scanning Tunneling Microscopy Investigation of Self-Assembled $\mathrm{CuPc} / \mathrm{F}{ }_{16} \mathrm{CuPc}$ Binary Superstructures on Graphite. Langmuir 2010, 26 (5), 3329-3334.

(354) Schull, G.; Douillard, L.; Fiorini-Debuisschert, C.; Charra, F.; Mathevet, F.; Kreher, D.; Attias, A.-J. Single-Molecule Dynamics in a Self-Assembled 2D Molecular Sieve. Nano Lett. 2006, 6 (7), 1360-1363. 
(355) Bobisch, C.; Wagner, T.; Bannani, A.; Möller, R. Ordered Binary Monolayer Composed of Two Organic Molecules: Copper-Phthalocyanine and 3,4,9,10-Perylene-Tetra-CarboxylicDianhydride on $\mathrm{Cu}(111)$. J. Chem. Phys. 2003, 119 (18), 9804-9808.

(356) Scudiero, L.; Hipps, K. W.; Barlow, D. E. A Self-Organized Two-Dimensional Bimolecular Structure. J. Phys. Chem. B 2003, 107 (13), 2903-2909.

(357) Hipps, K. W.; Scudiero, L.; Barlow, D. E.; Cooke, M. P. A Self-Organized 2Dimensional Bifunctional Structure Formed by Supramolecular Design. J. Am. Chem. Soc. 2002, 124 (10), 2126-2127.

(358) Lu, X.; Hipps, K. W.; Wang, X. D.; Mazur, U. Scanning Tunneling Microscopy of Metal Phthalocyanines: d7 and d9 Cases. J. Am. Chem. Soc. 1996, 118 (30), 7197-7202.

(359) de Wild, M.; Berner, S.; Suzuki, H.; Yanagi, H.; Schlettwein, D.; Ivan, S.; Baratoff, A.; Guentherodt, H.; Jung, T. A. A Novel Route To Molecular Self-Assembly: Self-Intermixed Monolayer Phases. ChemPhysChem 2002, 3 (10), 881-885.

(360) Berner, S.; Brunner, M.; Ramoino, L.; Suzuki, H.; Güntherodt, H.-J.; Jung, T. . Time Evolution Analysis of a 2D Solid-gas Equilibrium: A Model System for Molecular Adsorption and Diffusion. Chem. Phys. Lett. 2001, 348 (3-4), 175-181.

(361) Mannsfeld, S.; Reichhard, H.; Fritz, T. LEED and STM Investigation of Chloro(subphthalocyaninato)boron on Au(1 11 1). Surf. Sci. 2003, 525 (1-3), 215-221.

(362) Yanagi, H.; Ikuta, K.; Mukai, H.; Shibutani, T. STM-Induced Flip-Flop Switching of Adsorbed Subphthalocyanine Molecular Arrays. Nano Lett. 2002, 2 (9), 951-955.

(363) Ferraris, J.; Cowan, D. O.; Walatka, V.; Perlstein, J. H. Electron Transfer in a New Highly Conducting Donor-Acceptor Complex. J. Am. Chem. Soc. 1973, 95 (3), 948-949.

(364) Gonzalez-Lakunza, N.; Fernández-Torrente, I.; Franke, K.; Lorente, N.; Arnau, A.; Pascual, J. Formation of Dispersive Hybrid Bands at an Organic-Metal Interface. Phys. Rev. Lett. 2008, 100 (15), 156805.

(365) Fernandez-Torrente, I.; Monturet, S.; Franke, K.; Fraxedas, J.; Lorente, N.; Pascual, J. Long-Range Repulsive Interaction between Molecules on a Metal Surface Induced by Charge Transfer. Phys. Rev. Lett. 2007, 99 (17), 176103.

(366) Fernández-Torrente, I.; Franke, K. J.; Pascual, J. I. Vibrational Kondo Effect in Pure Organic Charge-Transfer Assemblies. Phys. Rev. Lett. 2008, 101 (21).

(367) Smerdon, J. A.; Rankin, R. B.; Greeley, J. P.; Guisinger, N. P.; Guest, J. R. Chiral "Pinwheel" Heterojunctions Self-Assembled from C 60 and Pentacene. ACS Nano 2013, 7 (4), 3086-3094.

(368) Lin Zhang, J.; Hong Liang Zhang, K.; Qiang Zhong, J.; Chao Niu, T.; Chen, W. LowTemperature Scanning Tunneling Microscopy/Ultraviolet Photoelectron Spectroscopy Investigation of Two-Dimensional Crystallization of C60: Pentacence Binary System on Ag(111). J. Appl. Phys. 2012, 111 (3), 34304. 
(369) Hooks, D. E.; Fritz, T.; Ward, M. D. Epitaxy and Molecular Organization on Solid Substrates. Adv. Mater. 2001, 13 (4), 227-241.

(370) Palma, C.-A.; Cecchini, M.; Samorì, P. Predicting Self-Assembly: From Empirism to Determinism. Chem. Soc. Rev. 2012, 41 (10), 3713.

(371) Mannsfeld, S. C. B.; Fritz, T. Analysis of the Substrate Influence on the Ordering of Epitaxial Molecular Layers: The Special Case of Point-on-Line Coincidence. Phys. Rev. B 2004, $69(7), 75416$.

(372) Mannsfeld, S. C. B.; Fritz, T. Understanding Organic-inorganic Heteroepitaxial Growth of Molecules on Crystalline Substrates: Experiment and Theory. Phys. Rev. B 2005, 71 (23), 235405 .

(373) Mannsfeld, S. C. B.; Leo, K.; Fritz, T. Line-on-Line Coincidence: A New Type of Epitaxy Found in Organic-Organic Heterolayers. Phys. Rev. Lett. 2005, 94 (5), 56104.

(374) Zhao, Y.; Wu, Q.; Chen, Q.; Wang, J. Molecular Self-Assembly on Two-Dimensional Atomic Crystals: Insights from Molecular Dynamics Simulations. J. Phys. Chem. Lett. 2015, 6 (22), 4518-4524.

(375) Brooks, B. R.; Bruccoleri, R. E.; Olafson, B. D.; States, D. J.; Swaminathan, S.; Karplus, M. CHARMM: A Program for Macromolecular Energy, Minimization, and Dynamics Calculations. J. Comput. Chem. 1983, 4 (2), 187-217.

(376) Brooks, B. R.; Brooks, C. L.; Mackerell, A. D.; Nilsson, L.; Petrella, R. J.; Roux, B.; Won, Y.; Archontis, G.; Bartels, C.; Boresch, S.; et al. CHARMM: The Biomolecular Simulation Program. J. Comput. Chem. 2009, 30 (10), 1545-1614.

(377) Palma, C.-A.; Samorì, P.; Cecchini, M. Atomistic Simulations of 2D Bicomponent SelfAssembly: From Molecular Recognition to Self-Healing. J. Am. Chem. Soc. 2010, 132 (50), $17880-17885$.

(378) Ciesielski, A.; Cadeddu, A.; Palma, C.-A.; Gorczyński, A.; Patroniak, V.; Cecchini, M.; Samorì, P. Self-Templating 2D Supramolecular Networks: A New Avenue to Reach Control over a Bilayer Formation. Nanoscale 2011, 3 (10), 4125-4129.

(379) Taylor, J. B.; Beton, P. H. Kinetic Instabilities in the Growth of One Dimensional Molecular Nanostructures. Phys. Rev. Lett. 2006, 97 (23), 236102.

(380) Li, Y.; Lin, N. Combined Scanning Tunneling Microscopy and Kinetic Monte Carlo Study on Kinetics of Cu-Coordinated Pyridyl-Porphyrin Supramolecular Self-Assembly on a Au(111) Surface. Phys. Rev. B 2011, 84 (12), 125418.

(381) van der Lit, J.; Marsman, J. L.; Koster, R. S.; Jacobse, P. H.; den Hartog, S. A.; Vanmaekelbergh, D.; Klein Gebbink, R. J. M.; Filion, L.; Swart, I. Modeling the Self-Assembly of Organic Molecules in 2D Molecular Layers with Different Structures. J. Phys. Chem. C 2016, 120 (1), 318-323. 
(382) Kasperski, A.; Szabelski, P. Theoretical Modeling of the Formation of Chiral Molecular Patterns in Self-Assembled Overlayers. Surf. Sci. 2014, 629, 57-64.

(383) Fortuna, S.; Cheung, D. L.; Johnston, K. Phase Behaviour of Self-Assembled Monolayers Controlled by Tuning Physisorbed and Chemisorbed States: A Lattice-Model View. J. Chem. Phys. 2016, 144 (13), 134707.

(384) Fortuna, S.; Cheung, D. L.; Troisi, A. Hexagonal Lattice Model of the Patterns Formed by Hydrogen-Bonded Molecules on the Surface. J. Phys. Chem. B 2010, 114 (5), 1849-1858.

(385) Weber, U.; Burlakov, V.; Perdigão, L.; Fawcett, R.; Beton, P.; Champness, N.; Jefferson, J.; Briggs, G.; Pettifor, D. Role of Interaction Anisotropy in the Formation and Stability of Molecular Templates. Phys. Rev. Lett. 2008, 100 (15), 156101.

(386) Palma, C.-A.; Bjork, J.; Bonini, M.; Dyer, M. S.; Llanes-Pallas, A.; Bonifazi, D.; Persson, M.; Samorì, P. Tailoring Bicomponent Supramolecular Nanoporous Networks: Phase Segregation, Polymorphism, and Glasses at the Solid-Liquid Interface. J. Am. Chem. Soc. 2009, 131 (36), 13062-13071.

(387) Nirmalraj, P. N.; Thompson, D.; Riel, H. E. Capturing the Embryonic Stages of SelfAssembly - Design Rules for Molecular Computation. Sci. Rep. 2015, 5, 10116.

(388) Marrink, S. J.; Risselada, H. J.; Yefimov, S.; Tieleman, D. P.; de Vries, A. H. The MARTINI Force Field: Coarse Grained Model for Biomolecular Simulations. J. Phys. Chem. B 2007, 111 (27), 7812-7824.

(389) Gobbo, C.; Beurroies, I.; de Ridder, D.; Eelkema, R.; Marrink, S. J.; De Feyter, S.; van Esch, J. H.; de Vries, A. H. MARTINI Model for Physisorption of Organic Molecules on Graphite. J. Phys. Chem. C 2013, 117 (30), 15623-15631.

(390) Kleppmann, N.; Klapp, S. H. L. A Scale-Bridging Modeling Approach for Anisotropic Organic Molecules at Patterned Semiconductor Surfaces. ArXiv14076265 Cond-Mat 2014.

(391) Roussel, T. J.; Vega, L. F. Modeling the Self-Assembly of Nano Objects: Applications to Supramolecular Organic Monolayers Adsorbed on Metal Surfaces. J. Chem. Theory Comput. 2013, 9 (5), 2161-2169.

(392) Roussel, T. J.; Barrena, E.; Ocal, C.; Faraudo, J. Predicting Supramolecular SelfAssembly on Reconstructed Metal Surfaces. Nanoscale 2014, 6 (14), 7991-8001.

(393) Llanes-Pallas, A.; Matena, M.; Jung, T.; Prato, M.; Stöhr, M.; Bonifazi, D. Trimodular Engineering of Linear Supramolecular Miniatures on $\operatorname{Ag}(111)$ Surfaces Controlled by Complementary Triple Hydrogen Bonds. Angew. Chem. Int. Ed. 2008, 47 (40), 7726-7730.

(394) Gardener, J. A.; Shvarova, O. Y.; Briggs, G. A. D.; Castell, M. R. Intricate HydrogenBonded Networks: Binary and Ternary Combinations of Uracil, PTCDI, and Melamine. J. Phys. Chem. C 2010, 114 (13), 5859-5866. 
(395) Such, B.; Trevethan, T.; Glatzel, T.; Kawai, S.; Zimmerli, L.; Meyer, E.; Shluger, A. L.; Amijs, C. H. M.; de Mendoza, P.; Echavarren, A. M. Functionalized Truxenes: Adsorption and Diffusion of Single Molecules on the $\operatorname{KBr}(001)$ Surface. ACS Nano 2010, 4 (6), 3429-3439.

(396) Kunstmann, T.; Schlarb, A.; Fendrich, M.; Wagner, T.; Möller, R.; Hoffmann, R. Dynamic Force Microscopy Study of 3,4,9,10-Perylenetetracarboxylic Dianhydride on KBr(001). Phys. Rev. B 2005, 71 (12), 121403.

(397) Loske, F.; Reichling, M.; Kühnle, A. Deposition Sequence Determines Morphology of C60 and 3,4,9,10-Perylenetetracarboxylic Diimide Islands on CaF2(111). Jpn. J. Appl. Phys. 2011, 50 (8), 08LB07.

(398) Burke, S. A.; Mativetsky, J. M.; Hoffmann, R.; Grütter, P. Nucleation and Submonolayer Growth of C_\{60\} on KBr. Phys. Rev. Lett. 2005, 94 (9), 96102.

(399) Hinaut, A.; Lekhal, K.; Aivazian, G.; Bataillé, S.; Gourdon, A.; Martrou, D.; Gauthier, S. NC-AFM Study of the Adsorption of Hexamethoxytriphenylene on $\operatorname{KBr}(001)$. J Phys Chem C 2011, 115 (27), 13338-13342.

(400) Hinaut, A.; Pujol, A.; Chaumeton, F.; Martrou, D.; Gourdon, A.; Gauthier, S. An NCAFM and KPFM Study of the Adsorption of a Triphenylene Derivative on $\mathrm{KBr}(001)$. Beilstein J. Nanotechnol. 2012, 3, 221-229.

(401) Kittelmann, M.; Rahe, P.; Gourdon, A.; Kühnle, A. Direct Visualization of Molecule Deprotonation on an Insulating Surface. ACS Nano 2012, 6 (8), 7406-7411.

(402) Baris, B.; Jeannoutot, J.; Luzet, V.; Palmino, F.; Rochefort, A.; Chérioux, F. Noncovalent Bicomponent Self-Assemblies on a Silicon Surface. ACS Nano 2012, 6 (8), 6905-6911.

(403) Zwaneveld, N. A. A.; Pawlak, R.; Abel, M.; Catalin, D.; Gigmes, D.; Bertin, D.; Porte, L. Organized Formation of 2D Extended Covalent Organic Frameworks at Surfaces. J. Am. Chem. Soc. 2008, 130 (21), 6678-6679.

(404) Blunt, M. O.; Russell, J. C.; Champness, N. R.; Beton, P. H. Templating Molecular Adsorption Using a Covalent Organic Framework. Chem. Commun. 2010, 46 (38), 7157-7159.

(405) Zhang, X.; Li, S.-S.; Chen, T.; Wang, D.; Wan, L.-J. Molecular Templates for Controlling and Ordering Organic Molecules on Solid Surfaces. Nano 2012, 7 (1), 1230001-1.

(406) Karamzadeh, B.; Eaton, T.; Cebula, I.; Torres, D. M.; Neuburger, M.; Mayor, M.; Buck, M. Bestowing Structure upon the Pores of a Supramolecular Network. Chem. Commun. 2014, 50 (91), 14175-14178.

(407) Kudernac, T.; Lei, S.; Elemans, J. A. A. W.; De Feyter, S. Two-Dimensional Supramolecular Self-Assembly: Nanoporous Networks on Surfaces. Chem. Soc. Rev. 2009, 38 (2), 402-421.

(408) Slater, A. G.; Beton, P. H.; Champness, N. R. Two-Dimensional Supramolecular Chemistry on Surfaces. Chem Sci 2011, 2 (8), 1440-1448. 
(409) Phillips, A. G.; Perdigão, L. M. A.; Beton, P. H.; Champness, N. R. Tailoring Pores for Guest Entrapment in a Unimolecular Surface Self-Assembled Hydrogen Bonded Network. Chem. Commun. 2010, 46 (16), 2775-2777.

(410) Zhang, J. L.; Zhong, S.; Zhong, J. Q.; Niu, T. C.; Hu, W. P.; Wee, A. T. S.; Chen, W. Rational Design of Two-Dimensional Molecular Donor-acceptor Nanostructure Arrays. Nanoscale 2015, 7 (10), 4306-4324.

(411) Kiebele, A.; Bonifazi, D.; Cheng, F.; Stöhr, M.; Diederich, F.; Jung, T.; Spillmann, H. Adsorption and Dynamics of Long-Range Interacting Fullerenes in a Flexible, TwoDimensional, Nanoporous Porphyrin Network. ChemPhysChem 2006, 7, 1462-1470.

(412) Bonifazi, D.; Kiebele, A.; Stöhr, M.; Cheng, F.; Jung, T.; Diederich, F.; Spillmann, H. Supramolecular Nanostructuring of Silver Surfaces via Self-Assembly of [60]Fullerene and Porphyrin Modules. Adv. Funct. Mater. 2007, 17 (7), 1051-1062.

(413) Samuely, T.; Liu, S.-X.; Haas, M.; Decurtins, S.; Jung, T. A.; Stöhr, M. Self-Assembly of Individually Addressable Complexes of $\mathrm{C}_{60}$ and Phthalocyanines on a Metal Surface: Structural and Electronic Investigations. J. Phys. Chem. C 2009, 113 (45), 19373-19375.

(414) Zhang, H. L.; Chen, W.; Chen, L.; Huang, H.; Wang, X. S.; Yuhara, J.; Wee, A. T. S. C60 Molecular Chains on $\alpha$-Sexithiophene Nanostripes. Small 2007, 3 (12), 2015-2018.

(415) Wang, R.; Mao, H. Y.; Huang, H.; Qi, D. C.; Chen, W. Scanning Tunneling Microscopy and Photoelectron Spectroscopy Investigation of the sexithiophene:C60 Donor-Acceptor Nanostructure Formation on Graphite. J. Appl. Phys. 2011, 109 (8), 84307.

(416) Huang, H.; Chen, W.; Chen, L.; Zhang, H. L.; Wang, X. S.; Bao, S. N.; Wee, A. T. S. "Zigzag" C[sub 60] Chain Arrays. Appl. Phys. Lett. 2008, 92 (2), 23105.

(417) Chen, L.; Chen, W.; Huang, H.; Zhang, H. L.; Yuhara, J.; Wee, A. T. S. Tunable Arrays of C60 Molecular Chains. Adv. Mater. 2008, 20 (3), 484-488.

(418) Sedona, F.; Di Marino, M.; Sambi, M.; Carofiglio, T.; Lubian, E.; Casarin, M.; Tondello, E. Fullerene/Porphyrin Multicomponent Nanostructures on Ag(110): From Supramolecular SelfAssembly to Extended Copolymers. ACS Nano 2010, 4 (9), 5147-5154.

(419) Vijayaraghavan, S.; Écija, D.; Auwärter, W.; Joshi, S.; Seufert, K.; Seitsonen, A. P.; Tashiro, K.; Barth, J. V. Selective Supramolecular Fullerene-Porphyrin Interactions and Switching in Surface-Confined C60-Ce(TPP)2 Dyads. Nano Lett. 2012, 12 (8), 4077-4083.

(420) Glowatzki, H.; Bröker, B.; Blum, R.-P.; Hofmann, O. T.; Vollmer, A.; Rieger, R.; Müllen, K.; Zojer, E.; Rabe, J. P.; Koch, N. "Soft" Metallic Contact to Isolated C ${ }_{60}$ Molecules. Nano Lett. 2008, 8 (11), 3825-3829.

(421) Xu, B.; Tao, C.; Williams, E. D.; Reutt-Robey, J. E. Coverage Dependent Supramolecular Structures: C60:ACA Monolayers on Ag(111). J. Am. Chem. Soc. 2006, 128 (26), 8493-8499. 
(422) Wei, Y.; Reutt-Robey, J. E. Directed Organization of C 70 Kagome Lattice by Titanyl Phthalocyanine Monolayer Template. J. Am. Chem. Soc. 2011, 133 (39), 15232-15235.

(423) Huang, H.; Wong, S. L.; Chen, W.; Wee, A. T. S. LT-STM Studies on SubstrateDependent Self-Assembly of Small Organic Molecules. J. Phys. Appl. Phys. 2011, 44 (46), 464005 .

(424) Fendrich, M.; Wagner, T.; Stöhr, M.; Möller, R. Hindered Rotation of a Copper Phthalocyanine Molecule on C60: Experiments and Molecular Mechanics Calculations. Phys. Rev. B 2006, 73 (11), 115433.

(425) Schmitz-Hübsch, T.; Sellam, F.; Staub, R.; Törker, M.; Fritz, T.; Kübel, C.; Müllen, K.; Leo, K. Direct Observation of Organic-organic Heteroepitaxy: Perylene-TetracarboxylicDianhydride on Hexa-Peri-Benzocoronene on Highly Ordered Pyrolytic Graphite. Surf. Sci. 2000, 445 (2), 358-367.

(426) Chen, W.; Huang, H.; Chen, S.; Chen, L.; Zhang, H. L.; Gao, X. Y.; Wee, A. T. S. Molecular Orientation of 3, 4, 9, 10-Perylene-Tetracarboxylic-Dianhydride Thin Films at Organic Heterojunction Interfaces. Appl. Phys. Lett. 2007, 91 (11), 114102.

(427) Huang, Y. L.; Chen, W.; Wee, A. T. S. Molecular Trapping on Two-Dimensional Binary Supramolecular Networks. J. Am. Chem. Soc. 2011, 133 (4), 820-825.

(428) Stadtmüller, B.; Willenbockel, M.; Schröder, S.; Kleimann, C.; Reinisch, E. M.; Ules, T.; Soubatch, S.; Ramsey, M. G.; Tautz, F. S.; Kumpf, C. Modification of the PTCDA-Ag Bond by Forming a Heteromolecular Bilayer Film. Phys. Rev. B 2015, 91 (15), 155433.

(429) Stadtmüller, B.; Lüftner, D.; Willenbockel, M.; Reinisch, E. M.; Sueyoshi, T.; Koller, G.; Soubatch, S.; Ramsey, M. G.; Puschnig, P.; Tautz, F. S.; et al. Unexpected Interplay of Bonding Height and Energy Level Alignment at Heteromolecular Hybrid Interfaces. Nat Commun 2014, 5,3685 . 


\section{Tables of Contents Graphic}

Bicomponent supramolecular architectures at the vacuum-solid interface Xavier Bouju†, Cristina Mattioli†, Grégory Franc†, Adeline Pujolł̦, André Gourdon†** $\dagger$ CEMES-CNRS, 29 Rue J. Marvig, 31055, Toulouse, France

$\$$ Université de Toulouse, UPS, CNRS, CEMES, 118 route de Narbonne, 31062 Toulouse (France)

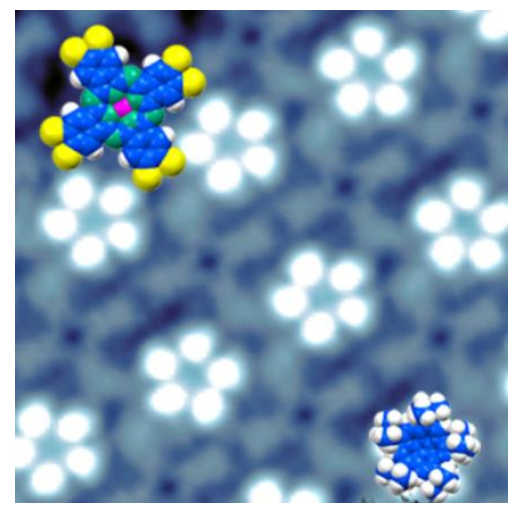

